\title{
How Well Can We Detect Shifts in Rates of Lineage Diversification? A Simulation Study of Sequential AIC Methods
}

R.H. MEDUSA Simulation Study

\author{
Michael R. MAY ${ }^{1}$ AND BRian R. MOORE $^{1}$ \\ ${ }^{1}$ Department of Evolution and Ecology, University of California, Davis \\ Davis, CA 95616, U.S.A.
}

Michael May

University of California, Davis

Department of Evolution and Ecology

Davis, CA 95616

U.S.A.

Phone: 530-752-7104

E-mail: mikeryanmay@gmail.com 
Abstract.-Evolutionary biologists have long been fascinated by the extreme differences in species numbers across branches of the Tree of Life. This has motivated the development of statistical phylogenetic methods for detecting shifts in the rate of lineage diversification (speciation - extinction). One of the most frequently used methods - implemented in the program MEDUSA - explores a set of diversification-rate models, where each model uniquely assigns branches of the phylogeny to a set of one or more diversification-rate categories. Each candidate model is first fit to the data, and the Akaike Information Criterion (AIC) is then used to identify the optimal diversification model. Surprisingly, the statistical behavior of this popular method is completely unknown, which is a concern in light of the poor performance of the AIC as a means of choosing among models in other phylogenetic comparative contexts, and also because of the ad hoc algorithm used to visit models. Here, we perform an extensive simulation study demonstrating that, as implemented, MEDUSA (1) has an extremely high Type I error rate (on average, spurious diversification-rate shifts are identified $42 \%$ of the time), and (2) provides severely biased parameter estimates (on average, estimated net-diversification and relative-extinction rates are $183 \%$ and $20 \%$ of their true values, respectively). We performed simulation experiments to reveal the source(s) of these pathologies, which include (1) the use of incorrect critical thresholds for model selection, and (2) errors in the likelihood function. Understanding the statistical behavior of MEDUSA is critical both to empirical researchers - in order to clarify whether these methods can reliably be applied to empirical datasets - and to theoretical biologists - in order to clarify whether new methods are required, and to reveal the specific problems that need to be solved in order to develop more reliable approaches for detecting shifts in the rate of lineage diversification.

[lineage-specific diversification rates, speciation, extinction, Akaike information criterion, phylogenetic model selection] 
Many evolutionary phenomena entail differential rates of diversification (speciation - extinction); e.g., adaptive radiation, diversity-dependent diversification, key innovations, and mass extinction. Phylogeny-based statistical methods have been developed to detect shifts in diversification rate through time, such as tree-wide shifts in diversification rate associated with episodes of mass extinction or adaptive radiation (Stadler 2010; 2011, Morlon et al. 2011), or diversity-dependent decreases in diversification rate associated with ecological limits on speciation (Rabosky 2006, Etienne et al. 2012). Other methods seek to identify correlations between rates of diversification and some other variable, such as the evolution of discrete or continuous traits (Maddison et al. 2007; FitzJohn 2010) or episodes of biogeographic or climatic change (Moore and Donoghue 2009; Goldberg et al. 2011). Here, we focus on a third class of methods that seek to detect shifts in diversification rate along lineages of a phylogenetic tree (Moore et al. 2004, Chan and Moore 2005; Rabosky et al. 2007; Alfaro et al. 2009).

The study of diversification-rate shifts along lineages is most often pursued using the approach proposed by Alfaro et al. (2009). In fact, this approach-Modeling Evolutionary Diversification Using Stepwise AIC (MEDUSA) — is used more frequently thanany other statistical phylogenetic method associated with lineage diversification (S.3). This approach assumes that phylogenies are generated under a birth-death stochastic-branching process model with one or more diversification-rate categories. The MEDUSA algorithm proceeds by first fitting a series of increasingly complex models with $1,2, \ldots, j$ diversification-rate categories; each with unique speciation, $\lambda$, and extinction, $\mu$ rate parameters. Each diversification model uniquely specifies both the number of rate categories and the assignment of those rates to branches of the phylogeny. After identifying the best diversification model for each category, MEDUSA then selects among the $j$ diversification models using standard model-selection methods (the Akaike Information Criterion, AIC; Akaike 1974).

The popularity of MEDUSA stems from several advantages it holds over alternative approaches: (1) rather than requiring complete species-level phylogenies, MEDUSA allows unsampled species to be included within unresolved terminal subclades; (2) rather than requiring the location of diversificationrate shifts to be specified a priori, MEDUSA agnostically evaluates diversification-rate shifts along all branches of the tree, allowing this problem to be studied within an exploratory-data analysis framework; (3) rather than assuming a pure-birth (Yule) stochastic-branching process model, MEDUSA is based on a more realistic birth-death model that accommodates extinction, and; (4) in addi- 
tion to inferring the location(s) of diversification-rate shifts, MEDUSA also provides estimates of the diversification-rate parameters for each branch of the tree.

Surprisingly, the statistical behavior of this popular method is completely unknown, and, in fact, the algorithm has never been formally described in the literature. This is particularly troubling, as the reliability of the AIC for model selection is known to be problematic in other phylogenetic contexts (Alfaro and Huelsenbeck 2006; Boettiger et al. 2012), and because three separate variants of the algorithm are now available to users. Simulation is a critical tool for validating an inference method, as it can uniquely assess the ability of an approach to recover the true/known parameter values that were used to generate the data. These controlled experiments allow us to characterize the statistical behavior of a given method. Simulation can either confirm that a method will provide reliable inferences when applied to empirical data (where the actual parameter values are unknown), or if it is found to be unreliable, simulation can be used to understand why the method fails.

With these considerations in mind, we first provide a formal description of the MEDUSA algorithm, and then perform an extensive simulation study to characterize the statistical behavior of the stepwise AIC method on simulated phylogenies. Our primary goals are (1) to reveal whether MEDUSA provides reliable estimates of diversification-rate shifts, and, if not (2) to understand why these methods are unreliable. Therefore, our simulation study is presented in the manner in which it was conducted: as an autopsy where (after determining that MEDUSA has fatal issues), we perform a series of simulation experiments to understand the nature of the methodological pathologies.

\section{INFERring DiversificAtion-RATE Shifts with MEDUSA}

\section{Data}

We treat the inferred phylogeny - including the tree topology and divergence times - as observations. Phylogenies may be complete, where each tip in the tree represents a single species. Alternatively, the phylogeny may be incomplete, in which case the tips represent terminally unresolved lineages. The latter species-diversity trees entail additional assumptions: the monophyly, age, and species diversity of all terminal lineages are assumed to be precisely known without error. More formally, the data comprise a rooted ultrametric tree with $k$ terminal lineages denoted as $\Psi=\{\tau, \mathbf{t}, \mathbf{n}\}$, where $\tau$ is the tree topology, $\mathbf{t}$ is a vector of divergence times starting from the root, and $\mathbf{n}$ is a vector of species-richness values for each of the $k$ terminal lineages. 


\section{Likelihood Function and Diversification-Rate Models}

We assume the phylogeny, $\Psi$, was generated by a birth-death stochastic-branching process with a vector of branch-specific speciation rates, $\boldsymbol{\lambda}=\left\{\lambda_{1}, \lambda_{2}, \ldots, \lambda_{2 k-2}\right\}$, and a vector of branch-specific extinction rates, $\boldsymbol{\mu}=\left\{\mu_{1}, \mu_{2}, \ldots, \mu_{2 k-2}\right\}$, where $\lambda_{i}$ and $\mu_{i}$ are the speciation and extinction rates for branch $i$, respectively.

Likelihood function.-The likelihood of observing the data under the stochastic-branching process model is calculated piecewise (c.f., Rabosky et al. 2007). We first compute the likelihood of the internal branches, then compute the likelihood of the terminal lineages, and finally combine these two partial likelihoods. The probability of observing internal branch of length $t_{i}$, conditional on it having descendants at the present, is

$$
P\left(t_{i} \mid \lambda_{i}, \mu_{i}\right)=\frac{\left(\lambda_{i}-\mu_{i}\right) \exp \left(-\left(\lambda_{i}-\mu_{i}\right) t_{i}\right)}{1-\frac{\mu_{i}}{\lambda_{i}} \exp \left(-\left(\lambda_{i}-\mu_{i}\right) t_{i}^{b}\right)}
$$

where $t_{i}^{b}$ is the birth-time of the branch (Nee et al. 1994; equation 17). The probability of observing terminal lineage $i$ with $n_{i}$ species, conditional on it having descendants at the present $\left(n_{i}>0\right)$ is

$$
P\left(n_{i} \mid t_{i}^{b}, \lambda_{i}, \mu_{i}\right)=\left(1-\frac{\exp \left(\left(\lambda_{i}-\mu_{i}\right) t_{i}^{b}\right)-1}{\exp \left(\left(\lambda_{i}-\mu_{i}\right) t_{i}^{b}\right)-\frac{\mu_{i}}{\lambda_{i}}}\right)\left(\frac{\exp \left(\left(\lambda_{i}-\mu_{i}\right) t_{i}^{b}\right)-1}{\exp \left(\left(\lambda_{i}-\mu_{i}\right) t_{i}^{b}\right)-\frac{\mu_{i}}{\lambda_{i}}}\right)^{n_{i}-1}
$$

which follows from (Raup 1985; equation A17). Parsing the tree into $\mathcal{T}$ terminal lineages and $\mathcal{I}$ internal branches, the likelihood for the phylogeny and species-richness data is calculated as

$$
P(\Psi \mid \boldsymbol{\lambda}, \boldsymbol{\mu})=\left(\prod_{i \in \mathcal{T}} P\left(t_{i} \mid t_{i}^{b}, \lambda_{i}, \mu_{i}\right)\right)\left(\prod_{i \in \mathcal{I}} P\left(n_{i} \mid t_{i}^{b}, \lambda_{i}, \mu_{i}\right)\right)
$$

as proposed by Rabosky et al. (2007).

Diversification-rate models.-The MEDUSA algorithm assumes that diversification-rate parameters are inherited identically over the tree unless a shift in diversification rate occurs along a branch. Every tree will have a "background" diversification rate (with a pair of rate parameters, $\lambda_{0}$ and $\mu_{0}$ ). Each of the $2 k-2$ branches on the tree can experience one or zero shifts in diversification rate. In principle, the diversification rate may change at any point(s) along a given branch. In practice, 
however, the MEDUSA algorithms assume that a single shift occurs either at the very beginning of a branch (i.e., immediately after the speciation event that gave rise to the branch), or at the very end of a branch (i.e., immediately before the branch experiences speciation). In the former 'stem' rate-shift scenario, the branch inherits the new diversification-rate parameters; in the latter 'crown' rate-shift scenario, the branch retains the ancestral diversification-rate parameters. Each of the $i$ rate shifts adds a new diversification-rate category (with rate parameters $\lambda_{i}$ and $\mu_{i}$ ). Models that share the same number of diversification-rate categories, $i$, belong to the same model index, which we denote $M_{i}$. Additionally, we use the notation $M_{i}^{j}$ to indicate the set of $j$ diversification-rate models that are members of model index $i$.

To illustrate our notation, consider the simple tree in Figure 1; the rooted, binary speciesdiversity tree has $k=4$ terminal lineages and $(2 k-2)=6$ branches. Accordingly, this tree can experience $i=\{0,1, \ldots, 4\}$ crown diversification-rate shifts, each with a corresponding model index $M_{i}$. Within a given model index, $M_{i}$, each of the $j$ distinct locations of the $i$ rate shifts on branches represents a unique diversification-rate model, $M_{i}^{j}$. For example, there is only a single model, $M_{0}^{1}$, where all branches diversify under the background diversification rate (with parameters $\lambda_{0}$ and $\mu_{0}$ ). By contrast, there are six distinct diversification-rate models (i.e., $M_{1}^{j}$, where $\left.j=\{1,2, \ldots, 6\}\right)$ that variously assign the single diversification-rate shift to one of the six branches in the tree.

The state space of possible diversification-rate models for a tree with $b=(2 k-2)$ branches is described by the corresponding Bell number (Bell 1934). The Bell number for $b$ branches is the sum of the Stirling numbers of the second kind:

$$
\mathcal{B}(b)=\sum_{k=1}^{b} \mathcal{S}_{2}(b, k) .
$$

The Stirling number of the second kind, $\mathcal{S}_{2}(b, k)$, for $b$ elements and $k$ subsets (corresponding here to the number of branches and diversification-rate categories, respectively) is given by the following equation:

$$
\mathcal{S}_{2}(b, k)=\frac{1}{k} \sum_{i=0}^{k-1}(-1)^{i}\left(\begin{array}{c}
k \\
i
\end{array}\right)(k-i)^{b} .
$$

The state space of possible diversification-rate models quickly becomes large, even for small trees. 
For example, the simple tree in Figure 1 has $\mathcal{B}(6)=203$ possible diversification-rate models, a species-diversity tree with six lineages has $\mathcal{B}(10)=115,975$ possible diversification-rate models, a tree with ten lineages has $\mathcal{B}(18)=682,076,806,174$ possible models, and a tree with 50 lineages has $\mathcal{B}(98)=3.12 \times 10^{112}$ possible models. Clearly, the space of candidate diversification models quickly becomes vast, which motivates the consideration of heuristic algorithms that might allow us to efficiently explore this space.

\section{The MEDUSA Algorithm}

The essence of the MEDUSA algorithm is straightforward: it explores the space of diversificationrate models, estimates the maximum likelihood for each candidate model, and then uses AIC to select the optimal diversification-rate model. The potentially vast solution space, however, requires heuristic techniques to efficiently search a small subspace of all possible diversification-rate models. An exhaustive algorithm (one that systematically evaluates every possible diversification-rate model) is prohibitive even for simple problems. Consider, for example, a species-diversity tree with ten lineages: even if we could compute the maximum-likelihood estimate for each candidate model at a rate of one per second, it would require $\sim 21,628$ years to evaluate every diversification-rate model. Although critical, heuristic shortcuts introduce considerable complexity into the MEDUSA algorithm. We provide a relatively detailed description of the MEDUSA algorithm in the Supplemental Material, and illustrate the basic procedure here with a simple example.

Consider the hypothetical species-diversity tree with four terminal lineages depicted in Figure 1. MEDUSA evaluates diversification-rate models in order of increasing complexity; $M_{0}, M_{1} \ldots, M_{\max }$. Accordingly, the algorithm begins by fitting a constant-rate model (i.e., $M_{0}$, with zero rate shifts) to the tree and species-richness data: numerical optimization is used to identify the rate-parameter values, $\hat{\lambda}_{0}$ and $\hat{\mu}_{0}$, that maximize the likelihood function (equation 3 . The resulting maximumlikelihood estimate, $\mathcal{L}$, under the one-rate model is then used to compute its AIC score: AIC $=2 p-2 \ln \mathcal{L}$, where $p$ is the number of free parameters in the model (Akaike 1974).

The MEDUSA algorithm then evaluates each of the diversification models with one rate-shift, $M_{1}^{j}$ (where $j=6$ diversification-rate models with two pairs of rate parameters, $\lambda_{0}, \mu_{0}$, and $\lambda_{1}, \mu_{1}$ ). The two-rate model that best fits the data (i.e., with the highest log likelihood) is selected, and its AIC score is computed. We then assess the relative improvement in the fit of the two-rate model over 
the one-rate model as the difference in their respective $\mathrm{AIC}$ scores: $\triangle \mathrm{AIC}=\mathrm{AIC}_{\text {one-rate }}-\mathrm{AIC}_{\text {two-rate }}$. If the computed $\triangle \mathrm{AIC}$ is greater than some pre-specified threshold, $\triangle \mathrm{AIC}_{\text {crit }}$, MEDUSA accepts the best two-rate model and the corresponding location of the diversification-rate shift specified by that two-rate model.

The algorithm then identifies the best three-rate model that incorporates the previously inferred rate shift, and accepts or rejects it over the two-rate model based on the same $\Delta \mathrm{AIC}_{\text {crit }}$ threshold. The algorithm continues, incrementing the model index until either (1) the most complex model is accepted or (2) the improvement in the AIC score is too small to exceed the $\Delta \mathrm{AIC}_{\text {crit }}$ threshold. In principle, the most complex model index is a function of the number of lineages, $M_{\max }=2 k-2$. In practice, however, $M_{\max }$ is set to an arbitrary value that limits the evaluation to a subset of all possible models (20 shifts in the original algorithm, but the number varies by implementation). Note that the MEDUSA algorithm tremendously reduces the scope of candidate diversification-rate models: in the present case - a species-diversity tree with four lineages - the space collapses from 203 to 16 candidate models.

Different implementations of MEDUSA specify the $\triangle \mathrm{AIC}_{\text {crit }}$ differently. The original implementation (Alfaro et al. 2009) uses the conventional value for the $\Delta \mathrm{AIC}_{\text {crit }}$ threshold, which is fixed at 4. More recent versions of the algorithm specify a critical threshold that is a function of the number of terminal lineages. Specifically, both turboMEDUSA (implemented both as a stand-alone R package and in GEIGER >1.99) and a newer version of MEDUSA (available at http://github.com/josephwb/tu rboMEDUSA) compute the $\triangle \mathrm{AIC}_{\text {crit }}$ threshold based on the number of terminal lineages (the latest versions prohibit negative $\Delta \mathrm{AIC}_{\text {crit }}$ values). An example of the three different $\Delta \mathrm{AIC}_{\text {crit }}$ thresholds (corresponding to the parameter space that we explore in one of our simulations) is depicted in Figure 3. We refer to the original MEDUSA, turboMEDUSA and the newest version of MEDUSA algorithms as oMEDUSA, tMEDUSA and nMEDUSA, respectively. 


\section{Simulation Study}

Our motivation for exploring the statistical behavior of the MEDUSA algorithm stems from its reliance on the Akaike Information Criterion (AIC) to choose among candidate diversification-rate models. Use of the AIC to choose among models in other phylogenetic settings is known to be problematic (e.g., Alfaro and Huelsenbeck 2006, Boettiger et al. 2012). Specifically, there is little theory to guide the specification of an appropriate critical threshold for preferring one model to another (i.e., the difference in $\mathrm{AIC}$ scores between the two competing models, $\Delta \mathrm{AIC}_{\text {crit }}$ ), and arbitrarily specified thresholds may strongly bias the model-selection procedure. Moreover, the AIC assumes that the sample size (in this case, the number of species/terminal lineages) is large. In practice, however, MEDUSA is generally applied to trees with a small number of incompletely sampled terminal lineages. Accordingly, the large-sample size assumption of the AIC is apt to be violated, which may compromise the ability of MEDUSA to reliably choose the correct model. With these considerations in mind, we performed a series of simulations to determine how well MEDUSA recovers the correct diversification-rate model under a variety of circumstances. All simulations were performed in $\mathrm{R}$ (R Core Team 2013) using the packages ape and TreeSim (Paradis et al. 2004; Stadler 2013); trees were subsequently manipulated with custom R scripts. All simulated data as well as the R scripts used to generate and analyze the simulated data are available from the Dryad Digital Repository: http://dx.doi.org/10.5061/dryad.261v1.

\section{Type I Error Rate}

We explored the Type I error rate of MEDUSA by simulating trees under a constant-rate birth-death process (i.e., where the diversification rate is constant across lineages), and then analyzed these trees with MEDUSA to assess the frequency with which it incorrectly inferred a diversification-rate shift (i.e., the false-positive rate). We simulated constant-rate trees over a wide range of diversification parameters (net diversification, $r=\lambda-\mu$, and relative extinction, $\epsilon=\mu \div \lambda$ ), tree sizes, $N$, and number of unresolved terminal lineages, $k$. The set of relative-extinction rates, $\epsilon$, that we explored span the possible range of values (from a pure-birth process with no extinction, to scenarios with an extreme extinction load). Our choice of parameters for tree size, $N$, and the number of terminally unresolved clades, $k$, reflects the dimensions of empirical datasets to which MEDUSA has been applied (Table S.1). We note that the algorithm as implemented in the most recent version GEIGER > 1.99, 
which most closely resembles tMEDUSA, is both considerably faster than oMEDUSA and is also the version recommended by the developers of these methods (Luke Harmon, personal communication). We have nevertheless opted to examine the behavior of all three iterations of the MEDUSA algorithm, as most of the empirical applications have used earlier versions of the method $(51.7 \%, 46.6 \%$, and $1.7 \%$ of empirical studies have used oMEDUSA, tMEDUSA and nMEDUSA, respectively; Table S.1. .

The effect of relative-extinction rate, tree size, and phylogenetic resolution. - In the first set of simulations, the absolute speciation rate was $\lambda=0.01$ and the extinction rate was incremented over a range of values, $\mu=\{0.000,0.001,0.002, \ldots, 0.009\}$ (see Table S.2 for corresponding composite parameters, $r$ and $\epsilon$ ). For each value of $\mu$, we simulated 1,000 trees of three sizes, $N=\{100,1,000,10,000\}$. We then created terminally unresolved clades in each tree by identifying the time when there were $k$ lineages, for $k=\{10,15,20,25,30,40,100\}$, pruning out all subsequent speciation events, and then assigning the corresponding species-richness values to each terminal lineage. In total, we generated 210, 000 trees with species-richness data $(1,000$ replicates for each combination of $\mu, N$, and $k$ ). We then analyzed each simulated tree using the three variants of the MEDUSA algorithm. Note that the original algorithm, oMEDUSA, assumes that diversification-rate shifts occur along stem branches, whereas the two more recent algorithms, tMEDUSA and nMEDUSA, allow the user to specify whether rate shifts are assumed to occur along crown or stem branches. To enhance comparability, we focus on results where we assumed that diversification-rate shifts occur along stem branches. However, we have replicated all of the analyses under the alternative option, where diversification-rate shifts were assumed to occur along crown branches (these results are presented in the Supplementary Material).

All three of the MEDUSA algorithms use the conventional optim algorithm (Nelder and Mead 1965) to estimate the vector of parameter values $(\boldsymbol{\lambda}$ and $\boldsymbol{\mu})$ that collectively maximize the likelihood of the phylogenetic observations, $\Psi$, under the corresponding diversification-rate model, $M_{i}^{j}$. In general, these numerical algorithms begin by first specifying an initial value for each parameter, and then calculate the log-likelihood for the vector of initial parameter values (by evaluating equation 3 ). New parameter values are iteratively proposed and evaluated until any possible change to parameter values decreases the log-likelihood, at which point the algorithm has either converged to a local or the global maximum-likelihood estimate, and the procedure terminates.

Our preliminary analyses using default initial parameter values revealed that the numerical al- 
gorithms used for maximum-likelihood estimation in MEDUSA frequently failed in one of two ways. Specifically, $25 \%$ of the analyses experienced ' $h a r d$ ' failures, where the program terminated with an error message indicating that the maximum log-likelihood could not be calculated. More troubling was the incidence of 'soft' failures, where analyses that appeared to terminate successfully actually failed to converge to the maximum-likelihood estimate. To control for the potentially confounding effect of this numerical instability on our evaluation of the statistical behavior of MEDUSA, we initialized analyses with parameters set to their true values (the parameter values used to simulate the data). Because the true parameter values are unknown for empirical datasets, our analyses of the simulated data will provide a relatively favorable assessment of the performance of the methods.

Results of the first simulation are summarized in Figure 2 (see also Table S.2). We can view Figure 2 as a graphical table with nine panels: the three panels in each row summarize results for one algorithm (oMEDUSA, tMEDUSA, and nMEDUSA, from top to bottom), and the three panels in each column summarize results for one tree size (with 100, 1,000, and 10,000 species, from left to right). The overall Type I error rate - calculated as the unweighted average over all of the cells within each of the nine panels - is $40.2 \%$, which is $\sim 8$ times higher than the nominal significance level, $\alpha=0.05$. The average Type I error rate varies over rows of Figure 2. 51\%, 29\%, 41\% for the progressively newer oMEDUSA, tMEDUSA, and nMEDUSA algorithms, respectively. Additionally, the overall Type I error rate tends to increase across each column of Figure 2. 26\%,47\%, $48 \%$ for increasingly larger trees.

The relatively clear patterns manifest among columns/rows of panels in Figure 2 contrasts with the complex patterns of Type I error rate observed within each of the panels. That is, trends in the Type I error rate within panels differed qualitatively between different versions of the algorithm. For example, Type I error rates for tMEDUSA tended to increase with the relative-extinction rate, $\epsilon$, and decrease with the number of terminal lineages, $k$, (increasing from the lower right to the upper left of each panel). By contrast, Type I error rates for nMEDUSA tended to increase with the relativeextinction rate and the number of terminal lineages (increasing from the lower left to the upper right of each panel). The source of the disparate behavior of the tMEDUSA and nMEDUSA algorithms is unclear, as they are said to be identical (Harmon et al. 2008), except that nMEDUSA does not allow negative $\Delta \mathrm{AIC}_{\text {crit }}$ values (c.f., Figure 3). Finally, patterns of Type I error rate within panels for oMEDUSA did not exhibit any clear correlation with the relative-extinction rate or the number of 
lineages, but appear to be some complex function of these two variables.

We first considered the possibility that the complex patterns of Type I error rate might be an artifact of Monte Carlo error (i.e., error variance caused by simulating insufficient replicates). However, our experiments using an increased number of replicates exclude this explanation; the high level and complex patterns of Type I error are estimated precisely (Figure S.4. Table S.3). Below, we describe our efforts to first rule out the possibility that the high Type I error rates are artifactual, and then to understand the possible source(s) of the high Type I error rates.

The effect of absolute diversification rates. - Could the high the Type I error rates inferred in the initial simulation be an artifact of the absolute parameter values that we used? Although our choice of diversification rates was based on a survey of 'normal' empirical values, it is possible that the MEDUSA algorithm might commonly be applied to groups with anomalously high diversification rates. To explore this possibility, we performed a second series of constant-rate simulations to assess the effect of increasing the absolute speciation rate, $\lambda$, on the statistical behavior of MEDUSA. Specifically, we simulated trees under a five- and ten-fold higher speciation rate, $\lambda=\{0.05,0.1\}$. For each speciation rate, we first simulated a set of trees under a range of extinction rates, $\mu$, such that the corresponding relative-extinction rates, $\epsilon$, were identical to those of the initial simulation; $\epsilon=\{0.0,0.1, \ldots, 0.9\}$ (Table S.4 . For each absolute speciation rate, we also simulated a second set of trees under a range of extinction rates, $\mu$, such that the corresponding net-diversification rates, $r$, were identical to those used in the initial simulation, $r=\{0.001,0.002, \ldots, 0.01\}$ (Table S.5). We simulated 1,000 trees with $N=10,000$ species for each combination of parameters. As in the initial simulation, we created a set of $k=\{10,15,20,25,30,40,100\}$ terminally unresolved lineages in each tree, resulting in $7 \times 4 \times 1,000=28,000$ trees with species-richness data. We then analyzed each tree using all three implementations of the MEDUSA algorithm. Again, we repeated the entire series of analyses evaluating 'stem' and 'crown' diversification-rate shifts.

Results of these simulations are summarized in Figures S.5 and S.6 (see also Tables S.4 S.5). Overall Type I error rates were slightly higher than those of the initial simulations: $40.2 \%, 44.7 \%$, and $44.7 \%$ for simulations based on the initial, five- and ten-fold higher absolute rates, respectively. The relative performance of the three MEDUSA algorithms was also similar to the initial simulation: oMEDUSA, tMEDUSA, and nMEDUSA exhibited Type I error rates of $44 \%, 40 \%$, and $51 \%$, respectively. Moreover, these simulations cast light on the impact of relative extinction. For the first set of higher- 
rate simulations - where the relative-extinction rates matched those of the initial simulation - the overall rate and patterns of Type I error were similar to those based on the initial lower-rate simulations (37.4\% vs. $40.2 \%$, respectively; Figure S.5). By contrast, the second set of higher-rate simulations - where the net-diversification rates matched those of the initial simulation - imposed a narrow range of relatively high relative-extinction rates, $\epsilon=\{0.80,0.82,0.84 \ldots, 0.98\}$. For these simulations, the overall Type I error rate increased substantially compared to the initial lower-rate simulation (51.5\% vs. $40.2 \%$, respectively; Figure S.6).

In summary, the high Type I error rates identified in the first simulation do not appear to be an artifact of the chosen parameter values, and appear to be strongly influenced by the severity of the relative-extinction rate.

Exploring threshold effects on Type I error rates. - Given that the high Type I error rates revealed in the previous simulations are real-they are not artifacts of Monte Carlo error or the specific parameter values that we used - we might ask whether these errors reflect an excess of "near misses", where there is only a slight preference for the (incorrect) multi-rate model over the (true) constantrate model. To explore this possibility, we examined the $\Delta \mathrm{AIC}$ values computed for the parameter space explored in the first simulation (Figure 3).

Surfaces of computed $\triangle \mathrm{AIC}$ values for the original algorithm, oMEDUSA, are both extreme and extremely complex. For smaller and intermediate trees, with $N=100$ and $N=1,000$ species, the computed $\triangle \mathrm{AIC}$ scores - reflecting the difference between the AIC scores for the (correct) rateconstant and the (incorrect) multi-rate models - increases as a complex function of the relativeextinction rate, $\epsilon$, and the number of terminal unresolved lineages, $k$. Moreover, the preference for the (incorrect) multi-rate model is typically very strong; the computed $\Delta \mathrm{AIC}$ values are up to 2500 times the fixed critical threshold (where $\Delta \mathrm{AIC}_{\text {crit }}=4$ ). Accordingly, the high Type I error rates for the oMEDUSA algorithm reflect a preponderance of "wild guesses" rather than "near misses". The pathological behavior of the oMEDUSA algorithm is compounded by the qualitatively different (but equally complex) surface of computed $\Delta \mathrm{AIC}$ values for the largest trees (with $N=10,000$ species). Clearly, the fixed threshold used by oMEDUSA is not a viable approach for assessing the relative fit of (and selecting among) diversification models.

Surfaces of computed $\triangle \mathrm{AIC}$ values for the newer tMEDUSA and nMEDUSA algorithms contrast sharply with the erratic behavior of the oMEDUSA algorithm. For small trees, with $N=100$ species, 
the computed $\triangle \mathrm{AIC}$ surfaces conform more uniformly to the corresponding critical thresholds. Accordingly, many of the false-positive cases based on tMEDUSA and nMEDUSA analyses of the smallest trees correspond to "near-ish misses", where the computed $\Delta \mathrm{AIC}$ moderately exceeds the $\Delta \mathrm{AIC}_{\text {crit }}$. By contrast, for larger trees, with $N=1,000$ and 10,000 species, surfaces of computed $\Delta$ AIC values for both algorithms greatly exceed the corresponding surface of critical thresholds, $\Delta \mathrm{AIC}_{\text {crit }}$. As was generally the case with the oMEDUSA algorithm, false-positive cases based on tMEDUSA and nMEDUSA analyses of larger trees represent "wild guesses", where the (true) constant-rate model is decisively rejected in favor of the (incorrect) multi-rate model.

In summary, support for the incorrect multi-rate model generally increases with tree size, $N$. This observation suggests two possible explanations. The first attributes the correlation between tree size and Type I error rate to power: constant-rate models may be rejected less frequently in small trees owing to a simple lack of statistical power to detect (spurious) diversification-rate shifts. The alternative explanation focuses on the relative proportion of the tree included in terminal unresolved lineages. Consider plots of the computed $\triangle$ AIC surfaces for tMEDUSA and MEDUSA (Figure 3). For smaller trees (with $N=100$ species), the degree to which the computed $\Delta$ AIC surface exceeds the $\Delta \mathrm{AIC}_{\text {crit }}$ threshold is a function of the number of terminal unresolved lineages, $k$. Note that we use the same set of terminal unresolved lineages in all simulations; $k=\{10,15,20,25,30,40,100\}$. The proportion of the tree relegated to terminal unresolved lineages therefore depends on tree size. For example, when $k=100$ and $N=100$, the tree is $100 \%$ resolved (each terminal lineage includes a single species), but when $k=100$ and $N=1,000$, the tree is only $10 \%$ resolved (900 species are apportioned among the 100 terminal unresolved lineages). These observations suggest that the high Type I error rate for the MEDUSA algorithms may be related to terminal unresolved lineages. We evaluate this possible explanation in the next section by estimating the Type I error rates for MEDUSA based on analyses of completely resolved trees.

Exploring Type I error rates for complete species trees.-In order to control for the apparently complex effects of terminally unresolved lineages on Type I error rate, we performed a series of simulations focused on complete species trees (i.e., trees in which each tip represents a single species). Specifically, we simulated complete trees of seven sizes, $N=\{50,100,150,200,250,500,1,000\}$, under a constant-rate birth-death process where the speciation rate, $\lambda$, was held at 0.01 , and the extinction rate, $\mu$, was incremented over a range of ten values, $\mu=\{0.000,0.001,0.002, \ldots, 0.009\}$. 
We simulated 1,000 replicates for each value of $N$ and $\mu$, generating a total of 70,000 complete species trees. We then analyzed each tree using tMEDUSA and nMEDUSA, and, as in previous simulations, repeated the entire series of analyses evaluating 'stem' and 'crown' diversification-rate shifts (we chose to omit oMEDUSA from these analyses because it proved to be prohibitively slow for completely sampled trees of these sizes).

Results for simulations based on completely sampled trees are summarized in Figure 4 (see also Table S.7). The overall Type I error rates were substantially lower in these simulations: tMEDUSA and nMEDUSA exhibited Type I error rates of $4 \%$ and $26 \%$, respectively. As in the previous simulations, the disparity in the behavior of the two newer MEDUSA algorithms is unexpected; we will return to this issue in the Discussion section. Importantly, the marked decrease in the overall Type I error rate for this set of simulations - which control for the effect of unresolved terminal cladesprovides further evidence that the pathological behavior of the MEDUSA methods stems either (1) from a problem computing the likelihood for terminal unresolved clades, and/or (2) from a problem combining the likelihoods for the terminal unresolved clades and the backbone tree. We explore the former possibility immediately below, and return to the latter possibility in the Discussion.

Assessing the impact of terminal unresolved lineages. - Could errors in calculating the likelihood of terminal unresolved clades be adversely impacting the performance of the MEDUSA algorithms? We explored this possibility by simulating trees with $N=\{1,2, \ldots, 10,20, \ldots, 100,200, \ldots, 1000\}$ species under a constant-rate birth-death process where the speciation rate was fixed to $\lambda=0.01$ and the extinction rate was incremented over a range of values, $\mu=\{0.000,0.001,0.002, \ldots, 0.009\}$. For each value of $\mu$ and $N$, we simulated 1,000 trees and recorded the stem age of each tree, $t$. We then used the stem age, $t$, and the size, $N$, of each tree-effectively treating it as a unresolved terminal lineage - to obtain maximum-likelihood estimates of $\lambda$ and $\mu$ (using equation 2 above, from Rabosky et al. 2007).

Results for simulations based on terminally unresolved lineages reveal that maximum-likelihood estimates of net-diversification rates and relative-extinction rates are strongly biased for very small lineages (Figure S.7. Tables S.8 and S.9. Not surprisingly, estimates become extremely biased when the terminal lineage includes a single species. Regardless of the true rates used to simulate these 'singleton' lineages $(N=1)$, the estimated net-diversification and relative-extinction rates must be zero, as there are no observed speciation (or extinction) events over the duration of the lineage, $t$. 
To assess whether these biased parameter estimates could impact Type I error rate, we explored the associated likelihood scores.

For each simulated tree, we computed the difference between the estimated log-likelihood of the data $\left(\ln \mathcal{L}_{\text {est }}\right.$, based on the estimated parameter values, $\hat{\lambda}$ and $\left.\hat{\mu}\right)$, and the actual log-likelihood of the data $\left(\ln \mathcal{L}_{\text {true }}\right.$, based on the generating parameter values, $\lambda$ and $\mu$ ):

$$
\Delta \ln \mathcal{L}=\ln \mathcal{L}_{\text {true }}-\ln \mathcal{L}_{\text {est }}
$$

More negative values of $\Delta \ln \mathcal{L}$ correspond to stronger support for a model that differs from the true model (note that $\Delta \ln \mathcal{L}$ can never be greater than zero). Echoing the bias in parameter estimates, singleton lineages exhibit strongly biased log-likelihood estimates. On average, the $\Delta \ln \mathcal{L}$ values for singleton lineages were nearly twice those of other lineages $\left({\overline{\Delta \ln \mathcal{L}_{N=1}}}=-1.01\right.$ compared to ${\overline{\Delta \ln \mathcal{L}_{N \neq 1}}}=-0.58 ;$ Figure 5]. This 'singleton effect' stems from instability in the likelihood function: for a tree with a single extant species, the maximum-likelihood estimate of the netdiversification rate converges to zero, and the likelihood of the data becomes 1 .

To assess whether this singleton effect contributes to the detection of spurious diversification-rate shifts, we explored the relationship between presence of singletons and patterns of Type I error in our simulated trees. For the initial constant-rate simulation, trees that were (incorrectly) identified by tMEDUSA and nMEDUSA to include a diversification-rate shift were significantly enriched for singletons ( $\chi^{2}$-test $p=0.012$ and $p \leq 10^{-16}$ for tMEDUSA and nMEDUSA, respectively); by contrast, oMEDUSA was significantly more likely to identify diversification-rate shifts in trees without singletons $\left(p \leq 10^{-16}\right)$. The relationship between tree size, resolution, relative extinction, and the enrichment of singletons among false-positive cases is complex (Figure S.8, Table S.10), but we note two general patterns: 1) in large regions of parameter space - especially when $N=10,000$ and $k$ is small-tMEDUSA and nMEDUSA always identify a spurious diversification-rate shift in the presence of a singleton, and; 2) the singleton effect decreases as phylogenetic resolution increases.

\section{Parameter Estimation}

The ability of MEDUSA to estimate parameters of the birth-death stochastic-branching process represents an important advantage over competing methods. This allows us to not only identify 
the number and phylogenetic distribution of diversification-rate shifts, but also to estimate the magnitude of corresponding changes in the net-diversification rate and/or relative-extinction rate. Although the intended focus of MEDUSA is estimating the location and number of diversificationrate shifts, it may also be used with a focus on estimating (absolute or relative) diversification rates. Most methods for estimating diversification rates assume that rates are constant through time and/or across lineages. However, estimates based on these methods are apt to be biased if the assumption of rate homogeneity is violated by shifts in diversification rate across lineages. In principle, MEDUSA can provide more reliable diversification-rate estimates by accommodating diversification-rate shifts as nuisance parameters. Accordingly, even if - as we have demonstrated above - MEDUSA does not provide reliable estimates of the location and number of diversificationrate shifts, it may nevertheless prove useful for estimating parameters of the birth-death process. In this section, we explore the ability of MEDUSA to provide reliable estimates of diversification-rate parameters.

Reliability of parameter estimates when the correct diversification model is selected.-How reliable are rate parameter estimates when MEDUSA selects the correct (constant-rate) diversification model? To explore this question, we examined parameter estimates for the cases in which MEDUSA identified the correct diversification model for trees simulated under a constant-rate birth-death process. On average, net-diversification rates estimated by oMEDUSA, tMEDUSA, and nMEDUSA were $479.7 \%, 113.0 \%$, and $106.5 \%$ their true values, respectively (Figures 6, S.21). Estimates of relative-extinction rate were similarly biased: parameter values inferred by oMEDUSA, tMEDUSA, and nMEDUSA were on average $6.7 \%, 20.2 \%$, and $27.8 \%$ their true values, respectively (Figures S.9. S.22). In summary, even when MEDUSA selects the correct diversification model, parameter estimates are severely biased.

Reliability of estimated magnitude of diversification-rate shifts.-Imagine that we have detected a significant diversification-rate shift using MEDUSA. Given the extremely high Type I error rate, we should be concerned that this diversification-rate shift is actually spurious. Imagine further, however, that the magnitude of the inferred diversification-rate shift is estimated to be very large. We might hope that the estimated magnitude could be used to help us distinguish between spurious and bona fide diversification-rate shifts. This would require that - despite severe bias in estimates of the absolute net-diversification and relative-extinction rates - estimates of the relative difference 
between these biased rate estimates prove to be reliable.

To explore this possibility, we plotted the magnitude of spurious diversification-rate shifts for the cases where MEDUSA incorrectly identified a multi-rate model in the constant-rate simulations. Spurious shifts in net-diversification rate were inferred to involve large average magnitudes (and broad 95\% quantiles): 2.3 (4.7), 2.7 (6.7), and 2.4 (4.8) for the oMEDUSA, tMEDUSA, and nMEDUSA algorithms, respectively (Figures 7, S.25, S.12, S.26). Similarly, spurious shifts in relative-extinction rate were inferred to have large average magnitudes (and wide 95\% quantiles): 2.7 (5.7), 3.1 (7.7), and 3.4 (7.9) for oMEDUSA, tMEDUSA, and nMEDUSA, respectively (Figures S.13, S.27, S.14, S.28). Accordingly, the magnitude of diversification-rate shifts estimated using MEDUSA are unreliable, and so cannot be used to distinguish 'real' from 'spurious' results when the method is applied to empirical data.

\section{DISCUSSION}

Biologists are clearly interested in identifying shifts in diversification rate along lineages, and have enthusiastically embraced MEDUSA as an approach for addressing this problem (Table S.1). MEDUSA has been used more frequently in empirical studies than any other statistical phylogenetic method for identifying lineage-specific diversification rates (Figure S.3), and, in fact, has been used more frequently than any statistical phylogenetic method for studying lineage diversification. Nevertheless, our simulation study reveals that MEDUSA is deeply flawed. In this section, we first summarize our inferences regarding the statistical behavior of MEDUSA, then consider the various pathologies that render this method unreliable, and conclude with a discussion of the more general implications of our findings.

\section{Characterizing the statistical (mis)behavior of MEDUSA}

For almost half of the trees that we simulated in our study - where rates were strictly constant across lineages of the tree-MEDUSA identified strong support for one or more diversification-rate shifts. This deeply troubling result holds for all versions of the algorithm (oMEDUSA, tMEDUSA, and nMEDUSA), applies to estimates derived using available options for these methods (whether rate shifts are assumed to occur along 'stem' or 'crown' nodes; see Supplemental Material), and holds over a wide range of absolute parameter values (Figures 2, S.5, S.6 S.17, S.18) that broadly encompass the conditions encountered in the analyses of actual empirical datasets (Figures S.1, S.2. Table S.1). 
The selection of (incorrect) multi-rate models by MEDUSA does not represent a slight bias. Rather, the (correct) constant-rate model is decisively rejected. Recall that the conventional threshold indicating a significant difference between two competing models is $\Delta \mathrm{AIC}_{\text {crit }}=4$. By contrast, the computed $\triangle \mathrm{AIC}$ values in favor of the incorrect diversification model exceeded the critical threshold of significance by an average of 522.7 AIC units (the oMEDUSA, tMEDUSA, and nMEDUSA algorithms had average values of 1035.5, 3.5, and 6.4, respectively; Figures 3. S.19).

We emphasize that these extreme false discovery rates are not artifacts of our simulation study. Our findings cannot be attributed to the optimization routine being trapped on local optima far away from global optima, as we initiated analyses of simulated trees from the true parameter values (those used to simulate the data). Our characterization of the statistical behavior of MEDUSA is therefore somewhat optimistic; the method will fare worse when applied to real datasets, where the true parameter values are unknown. Additionally, our conclusions are not manifestations of Monte Carlo error: we simulated more than twice the number of replicates required for precise estimates of the level and patterns of Type I error (Figures S.4, S.16, Tables S.3, S.20).

MEDUSA also provides severely biased estimates of diversification-rate parameters (Figures 6, 7). Even when MEDUSA correctly identified the (true) constant-rate model, the net-diversification and relative-extinction rate estimates were on average $183 \%$ and $20 \%$ of their true values, respectively (Figures 6, S.9, S.21, S.22). Similarly, when MEDUSA identified the (incorrect) multi-rate model, the spurious diversification-rate shifts were inferred to involve rate changes of large magnitude. On average, spurious diversification-rate shifts were estimated to involve a 2.5 -fold change in netdiversification rate (Figures 7, S.25), with a large spread of values (the upper $95 \%$ quantile was an average 5.5-fold shift in net-diversification rate; Figures S.12, S.26). Similarly, these illusory diversification-rate shifts were estimated to entail an average 3.1-fold change in relative-extinction rate (Figures S.13, S.27), with a correspondingly large spread of values (the upper $95 \%$ quantile was an average 7.4-fold shift in net-diversification rate; Figures S.14, S.28,

In summary, many of the putative advances that make MEDUSA appealing seem to be problematic. For example, the ability to accommodate incompletely sampled trees with MEDUSA is an attractive feature of the method; in fact, most applications (82.5\%) of this method involve incomplete trees. Nevertheless, the pathological behavior of MEDUSA is most extreme under these conditions (Figures 4. S.20). Likewise, the adoption of a birth-death branching process is presented as an important 
advantage of MEDUSA over competing methods that do not accommodate extinction. However, (relative) extinction-rate estimates obtained using MEDUSA are effectively meaningless (Figures S.9, S.11. S.22, S.24). This finding is relevant to the general debate regarding whether (relative) extinction rates can (e.g., Rabosky et al. 2007, Rabosky 2014) or cannot (e.g., Rabosky 2010) be reliably estimated from phylogenies. Our sense is that procuring reliable estimates of (relative) extinction rates from phylogenies of extant species is generally difficult when diversification rates are constant, and becomes extremely difficult when diversification rates experience tree-wide changes through time, and is effectively impossible (or at least inadvisable) when diversification rates change along lineages.

\section{Diagnosing the pathologies afflicting MEDUSA}

The pathological behavior of MEDUSA stems from two primary causes: the likelihood functions are incorrect, and the thresholds for evaluating the fit of candidate diversification models are unreliable. The likelihood function is critical to likelihood-based (maximum-likelihood and Bayesian inference) methods, as it is the vehicle that conveys information in the data to estimate the model parameters. Accordingly, even a minor error in the likelihood function of a method is likely to render it unreliable. The likelihood functions of MEDUSA contain two major flaws. First, the likelihood function used to compute the probability of terminal lineages (equation 2) is unstable. As we have demonstrated, this instability causes severely biased estimates of parameters (Figure S.7) and the corresponding log-likelihoods (Figure 5) for 'singleton' terminal lineages (i.e., those comprising a single species). Consequently, when MEDUSA is applied to a tree containing one or more terminal lineages with a single species, this 'singleton effect' creates the illusion of rate variation within the tree, which may cause overfitting of the diversification models. That is, the singleton effect contributes to the selection of diversification models that entail one or more spurious rate shifts.

Second, and more profoundly, MEDUSA is based on a composite likelihood function (equation 3 , Rabosky et al. 2007) that is invalid for trees in which diversification rates change across lineages. The expression for the composite likelihood (i.e., for the backbone, $\mathcal{I}$, and the terminal unresolved lineages, $\mathcal{T}$, of the tree) is only correct when rates of diversification are stochastically constant. The probabilities for the internal branches, $P\left(\tau_{i} \mid \cdot\right)$, and terminal unresolved polytomies, $P\left(n_{i} \mid \cdot\right)$, are both conditional on a homogeneous rate of speciation and extinction, $\lambda_{i}, \mu_{i}$, across the tree. Variation in diversification rates across branches and/or terminal lineages - the process that MEDUSA 
was designed to detect - clearly violates this condition and results in biased parameter estimates and incorrect likelihoods.

The inability of MEDUSA to correctly estimate the likelihood of a given diversification model is a fatal problem. However, MEDUSA is further confounded by problems associated with selecting among candidate diversification models. Recall that MEDUSA uses the AIC to select among competing diversification models: we first estimate the maximum-likelihood score, $\ln \mathcal{L}$, for two adjacent diversification models $-M_{i}$ and $M_{i+1}$ with $i$ and $i+1$ diversification-rate categories - then calculate the AIC score for each candidate model-AIC $=2 p-2 \ln \mathcal{L}$ with $p$ free parameters - and finally compute the difference in the $\mathrm{AIC}$ scores for the two competing models $-\Delta \mathrm{AIC}=\mathrm{AIC}_{i}-\mathrm{AIC}_{i+1}$. Finally, we compare the computed $\Delta \mathrm{AIC}$ score to the critical threshold, $\Delta \mathrm{AIC}_{\text {crit }}$; we reject the simpler model if the improvement in the AIC score conferred by the more complex model exceeds this threshold. The original implementation, oMEDUSA, used a conventional, fixed $\triangle \mathrm{AIC}_{\text {crit }}$ threshold. As we have shown (Figures 3, S.19), a fixed critical $\Delta \mathrm{AIC}_{\text {crit }}$ threshold is not a viable solution: the surface of computed $\triangle \mathrm{AIC}$ values varies considerably depending upon the tree size, $N$, the number of terminal unresolved lineages, $k$, and the relative-extinction rate, $\epsilon$. When the computed $\Delta$ AIC values exceed the fixed $\triangle \mathrm{AIC}_{\text {crit }}$ threshold, the MEDUSA will select the incorrect (overly complex) diversification model, and in so doing, identify spurious diversification-rate shifts.

Developers of MEDUSA also appear to have realized that a conventional, fixed $\Delta \mathrm{AIC}_{\text {crit }}$ threshold is not appropriate for selecting among diversification models (Luke Harmon, personal communication). As we understand it, they developed the following solution to this problem. First, they performed a simulation study that was similar in some respects to the current study: they simulated trees of various sizes, $N$, under a constant-rate birth-death process. Next, they generated the distribution of $\triangle \mathrm{AIC}$ values computed for one- versus two-rate diversification models for the various tree sizes. Finally, they devised a function that approximated the resulting distribution of computed $\Delta$ AIC values, which they use to specify the $\Delta \mathrm{AIC}_{\text {crit }}$ threshold values in the newer versions of the algorithm. This is the basis for the surface of $\mathrm{AIC}_{\text {crit }}$ values depicted in Figures 3 and $\mathrm{S.19}$.

Although clearly an improvement over the conventional, fixed $\Delta \mathrm{AIC}_{\text {crit }}$ critical threshold, this solution is still inadequate. The critical $\Delta \mathrm{AIC}_{\text {crit }}$ threshold used in the two newer algorithms, tMEDUSA and nMEDUSA, is strictly a static function of tree size, $N$, for completely sampled trees. As we have seen, however, computed $\triangle \mathrm{AIC}$ values are also strongly impacted by the degree of 
phylogenetic resolution (i.e., the number of terminal unresolved linages, $k$ ), the relative extinction rate, $\epsilon$, and the absolute diversification rate. Importantly, the static function implemented in tMEDUSA and nMEDUSA does not capture the effect of these factors on the computed $\triangle \mathrm{AIC}$ values. Accordingly, when the data depart from the precise conditions used to devise the static function, the critical $\Delta \mathrm{AIC}_{\text {crit }}$ threshold based on that static threshold becomes increasingly incorrect, and Type I error rate becomes increasingly inflated.

This insight suggests a possible solution: we simply need to devise a more complex static function that captures the impact of these additional factors on the computed $\Delta \mathrm{AIC}$ values. However, we believe that such an extension will not provide a viable solution. The static function used to describe the critical $\triangle \mathrm{AIC}_{\text {crit }}$ threshold in tMEDUSA and nMEDUSA is based on constant-rate simulations. Specifically, the function is attempting to describe the distribution of $\Delta \mathrm{AIC}$ values computed for the (correct) constant-rate versus the (incorrect) two-rate models, $\Delta \mathrm{AIC}=\mathrm{AIC}_{\text {one-rate }}-\mathrm{AIC}_{\text {two-rate }}$. Our current study focusses exclusively on simulations under a constant-rate birth-death process. However, our work on a related project involves an extensive simulation study of birth-death processes that involve one or more diversification-rate shifts (May, Höhna, and Moore, in preparation). We have discovered that the computed $\Delta$ AIC values depend on the absolute degree of the models being compared. That is, all else being equal, a critical $\Delta \mathrm{AIC}_{\text {crit }}$ threshold that is correct for selecting between diversification models with one- versus two-rate categories is not reliable for selecting between diversification models with two- versus three-rate categories.

Accordingly, providing a correct and general solution for robust selection of diversification models may require that we dynamically generate critical $\Delta \mathrm{AIC}_{\text {crit }}$ thresholds that are precisely tailored for the specific comparison at hand. We are optimistic that such a solution could be devised using Monte Carlo simulation; this is an area of current work (May, Höhna, and Moore, in preparation).

\section{General implications of the current study}

The problematic likelihood function used in MEDUSA (equation 3, Rabosky et al. 2007) is also used in - and may similarly compromise the reliability of - several other methods developed for estimating diversification rates from incompletely sampled phylogenies, including those in LASER Rabosky et al. 2007) and MECCA (Slater et al. 2012). Similarly, many statistical phylogenetic methods are based on selecting among candidate models using the Akaike Information Criterion. These include methods for studying lineage diversification (Rabosky 2006, Rabosky and Lovette 2008; Rabosky and Glor 
2010), and approaches for exploring the evolution of continuous traits under either Brownian motion (Thomas and Freckleton 2012) or Ornstein-Uhlenbeck (Ingram and Mahler 2013) process models. The AIC is also used to assess the relative fit of candidate models of nucleotide substitution (e.g., Posada 2008; Darriba et al. 2012), to select among partition schemes (e.g., Lanfear et al. 2012), and to choose among models of lineage-specific natural-selection pressure (e.g, Pond and Frost 2005). The AIC provides a seemingly attractive solution for choosing among models - it is fast and easy to compute, and can be flexibly applied to select among nested or non-nested models. However, our results accord with those of previous studies (e.g., Alfaro and Huelsenbeck 2006; Boettiger et al. 2012), suggesting that the AIC is not a reliable means for choosing among phylogenetic models.

Owing to the inherent complexity of the inference problems, the vast majority of comparative phylogenetic methods cannot be solved analytically, and so resort to numerical approximations (i.e., hill-climbing algorithms used for maximum-likelihood estimates of parameters or MCMC algorithms used in Bayesian methods to approximate the joint posterior probability density of parameters). Careful simulation is therefore required to validate these methods: this approach can uniquely assess the ability of the inference methods to recover the true (known) parameters and/or model used to generate the data. This is particularly true of ad hoc methods (i.e., those not developed within a formal modeling framework), which are becoming increasingly popular in the field of comparative phylogenetic: 1 ,

Accordingly, the design and execution of simulation studies deserve our careful consideration. A simulation study will provide meaningful insights into the statistical behavior of a given method only if it adequately explores the relevant parameter space. Conceptually, this entails circumscribing an $N$-dimensional hypervolume (for methods impacted by $N$ variables, such as tree size, diversification rate, etc.) that is likely to encompass the conditions encountered in the course of applying the method to real data. This hypervolume is discretized into cells, each representing a unique combination or parameter values. The granularity of the discretization required to faithfully characterize the statistical behavior of a method depends on its details. Methods developed in a formal modeling framework generally require a less granular discretization of parameter space, as their direct connection to established statistical theory provides a basis for interpolating their behavior

\footnotetext{
${ }^{1}$ MEDUSA is classified as an ad hoc method because it does not explicitly model changes in diversification rate over the tree as a stochastic process, but instead heuristically evaluates the assignment of diversification rates to branches of the tree according to an arbitrarily algorithm.
} 
between sampled points in parameter space. By contrast, ad hoc methods generally require a more finely discretized (and therefore more demanding) exploration of parameter space, as their behavior is apt to be relatively unpredictable. This behavioral inscrutability is manifest in the present study: e.g., Type I error rates and bias in parameter estimates varied wildly over parameter space (Figures 2, 3). Finally, each discrete cell of parameter space must be explored with sufficient intensity. Practically, this means simulating an adequate number of outcomes for each unique combination of parameter values to provide 'acceptable' error variance on the corresponding estimates. The degree of Monte Carlo error associated with a finite number of replicates can (and should) be assessed (c.f., Figure S.4. Careful simulation studies represent a non-trivial enterprise: our study of the MEDUSA algorithm involved $\sim 7,000,000$ analyses that consumed $\sim 35,000$ hours $(\sim 4$ years $)$ of CPU time.

From this perspective, our experience emphasizes that the increasingly popular use of $\mathrm{R}$ for developing comparative phylogenetic methods is a double-edged sword. The primary advantage is the very low threshold required to quickly implement a method, often by drawing upon existing $\mathrm{R}$ functions and packages. The downside, however, is that the extremely slow speed of methods implemented purely in $\mathrm{R}$ (which is typically $\sim 30-60$ times slower than low-level programming languages, such as $\mathrm{C}$ and $\mathrm{C++}$ ) is a major impediment to the necessary validation of these methods via simulation. For this reason, the statistical behavior of many recent comparative phylogenetic methods is largely (or completely) unknown. We believe this is a reason for serious concern.

The primary conclusion of our simulation study - that the MEDUSA methods are deeply flawed might be viewed as a 'negative' result. After all, our findings cast considerable doubt on the conclusions of the many empirical studies that have used MEDUSA, and argue strongly against the application of this approach in future empirical studies. Nevertheless, we believe that it is far better to be aware of the limitations of a method (even to the extent where it is deemed unusable) than to (unwittingly) continue using a method that is likely to provide spurious results. Moreover, beyond demonstrating that MEDUSA is unreliable, the results of our simulation study provide insights into the reasons why these methods are unreliable. Understanding the nature of these problems can focus theoretical efforts to develop more reliable methods. Accordingly, far from discouraging, we view the findings of this study as cause for optimism. We are hopeful that future efforts will resolve issues afflicting the MEDUSA framework for identifying shifts in diversification rates that - coupled with rigorous evaluation of these new methods - will continue to enhance our ability to explore a 
broad range of fundamental evolutionary processes.

\section{ACKNOWLEDGMENTS}

We are grateful to Michael Turelli and Peter Wainwright for encouraging us to pursue this project, to Yaniv Brandvain, Tracy Heath, Sebastian Höhna, John Huelsenbeck, Michael Landis, Bruce Rannala, and Tanja Stadler for thoughtful discussion, and to Andrew Magee for assistance with the survey of empirical studies. We are particularly grateful to Mike Alfaro and Luke Harmon for sharing their insights on this work. This research was supported by NSF grants DEB-0842181, DEB-0919529, and DBI-1356737 awarded to BRM. Computational resources for this work were provided by an NSF XSEDE grant (DEB-120031) to BRM. 


\section{REFERENCES}

Akaike, H. 1974. A new look at the statistical model identification. Automatic Control, IEEE Transactions on 19:716-723.

Alfaro, M. E. and J. P. Huelsenbeck. 2006. Comparative performance of Bayesian and AIC-based measures of phylogenetic model uncertainty. Systematic Biology 55:89-96.

Alfaro, M. E., F. Santini, C. Brock, H. Alamillo, A. Dornburg, D. L. Rabosky, G. Carnevale, and L. J. Harmon. 2009. Nine exceptional radiations plus high turnover explain species diversity in jawed vertebrates. Proceedings of the National Academy of Sciences 106:13410-13414.

Bell, E. T. 1934. Exponential numbers. American Mathematics Monthly 41:411-419.

Boettiger, C., G. Coop, and P. Ralph. 2012. Is your phylogeny informative? Measuring the power of comparative methods. Evolution 66:2240-2251.

Chan, K. M. A. and B. R. Moore. 2005. SymmeTREE: whole-tree analysis of differential diversification rates. Bioinformatics 21:1709-1710.

Darriba, D., G. L. Taboada, R. Doallo, and D. Posada. 2012. jmodeltest 2: more models, new heuristics and parallel computing. Nature Methods 9:772.

Etienne, R., B. Haegeman, T. Stadler, T. Aze, P. Pearson, A. Purvis, and A. Phillimore. 2012. Diversity-dependence brings molecular phylogenies closer to agreement with the fossil record. Proceedings of the Royal Society B: Biological Sciences 279:1300-1309.

FitzJohn, R. G. 2010. Quantitative traits and diversification. Systematic Biology 59:619-633.

Goldberg, E. E., L. T. Lancaster, and R. H. Ree. 2011. Phylogenetic inference of reciprocal effects between geographic range evolution and diversification. Systematic Biology Page in press.

Harmon, L., J. Weir, C. Brock, R. Glor, and W. Challenger. 2008. Geiger: investigating evolutionary radiations. Bioinformatics 24:129-131. 
Ingram, T. and D. Mahler. 2013. Surface: detecting convergent evolution from comparative data by fitting ornstein-uhlenbeck models with stepwise akaike information criterion. Methods in Ecology and Evolution 4:416-425.

Lanfear, R., B. Calcott, S. Y. W. Ho, and S. Guindon. 2012. Partitionfinder: Combined selection of partitioning schemes and substitution models for phylogenetic analyses. Molecular Biology and Evolution 29:1695-1701.

Leslie, A. B., J. M. Beaulieu, P. R. Crane, and M. J. Donoghue. 2013. Explaining the distribution of breeding and dispersal syndromes in conifers. Proceedings of the Royal Society B: Biological Sciences 280 .

Maddison, W., P. Midford, and S. Otto. 2007. Estimating a binary character's effect on speciation and extinction. Systematic Biology 56:701.

Moore, B. R., K. M. A. Chan, and M. J. Donoghue. 2004. Detecting diversification rate variation in supertrees. Pages 487-533 in Phylogenetic Supertrees: Combining Information to Reveal the Tree of Life (O. R. P. Bininda-Emonds, ed.) Kluwer Academic Press.

Moore, B. R. and M. J. Donoghue. 2009. A Bayesian approach for evaluating the impact of historical events on rates of diversification. Proceedings of the National Academy of Sciences 106:43074312.

Morlon, H., T. Parsons, and J. Plotkin. 2011. Reconciling molecular phylogenies with the fossil record. Proceedings of the National Academy of Sciences 108:16327-16332.

Nee, S., R. M. May, and P. H. Harvey. 1994. The reconstructed evolutionary process. Philosophical Transactions of the Royal Society B 344:305-311.

Nelder, J. A. and R. Mead. 1965. A simplex method for function minimization. The Computer Journal 7:308-313.

Paradis, E., J. Claude, and K. Strimmer. 2004. APE: analyses of phylogenetics and evolution in R language. Bioinformatics 20:289-290.

Pond, S. L. K. and S. D. W. Frost. 2005. A genetic algorithm approach to detecting lineage-specific variation in selection pressure. Molecular Biology and Evolution 22:478-485. 
Posada, D. 2008. jmodeltest: Phylogenetic model averaging. Molecular Biology and Evolution $25: 1253-1256$.

R Core Team. 2013. R: A Language and Environment for Statistical Computing. R Foundation for Statistical Computing Vienna, Austria ISBN 3-900051-07-0.

Rabosky, D. 2006. Likelihood methods for detecting temporal shifts in diversification rates. Evolution 60:1152-1164.

Rabosky, D. and I. Lovette. 2008. Explosive evolutionary radiations: decreasing speciation or increasing extinction through time? Evolution 62:1866-1875.

Rabosky, D. L. 2010. Extinction rates should not be estimated from molecular phylogenies. Evolution; international journal of organic evolution 64:1816-1824.

Rabosky, D. L. 2014. Automatic detection of key innovations, rate shifts, and diversity-dependence on phylogenetic trees. PLoS ONE 9:e89543.

Rabosky, D. L., S. C. Donnellan, A. L. Talaba, and I. J. Lovette. 2007. Exceptional among-lineage variation in diversification rates during the radiation of Australia's most diverse vertebrate clade. Proceedings of the Royal Society B: Biological Sciences 274:2915-2923.

Rabosky, D. L. and R. E. Glor. 2010. Equilibrium speciation dynamics in a model adaptive radiation of island lizards. Proceedings of the National Academy of Sciences 107:22178-22183.

Raup, D. M. 1985. Mathematical models of cladogenesis. Paleobiology 11:42-52.

Slater, G. J., L. J. Harmon, D. Wegmann, P. Joyce, L. J. Revell, and M. E. Alfaro. 2012. Fitting models of continuous trait evolution to incompletely sampled comparative data using approximate bayesian computation. Evolution 66:752-762.

Stadler, T. 2010. Sampling-through-time in birth-death trees. Journal of Theoretical Biology .

Stadler, T. 2011. Mammalian phylogeny reveals recent diversification rate shifts. Proceedings of the National Academy of Sciences, USA 108:6187-6192.

Stadler, T. 2013. TreeSim: Simulating trees under the birth-death model. R package version 1.8. 
bioRxiv preprint doi: https://doi.org/10.1101/011452; this version posted November 15,2014 . The copyright holder for this preprint (which was not certified by peer review) is the author/funder, who has granted bioRxiv a license to display the preprint in perpetuity. It is made available under aCC-BY-ND 4.0 International license.

Thomas, G. H. and R. P. Freckleton. 2012. Motmot: models of trait macroevolution on trees. Methods in Ecology and Evolution 3:145-151. 


\section{Figure LEGENDS}

Figure 1. The MEDUSA algorithm. 1) The one-rate model, $M_{1}$, is fit to the data using maximum likelihood. 2) Every possible two-rate model is then fit to the data, and the models are ranked by their likelihood score (with the best model, $M_{2}$, at the bottom). 3) Every possible three-rate model that is consistent with the best two-rate model is then fit to the data, and the models are then ranked as in the previous step. 4) Step 3 is repeated for increasingly complex models until the most complex model has been evaluated. 5) The AIC score is computed for the best model in each index (i.e., with $0,1, \ldots$ rate shifts). 6) The $\Delta \mathrm{AIC}$ value is calculated for each pair of adjacent models (as the difference in their AIC scores). Each of the $\Delta A I C_{i}$ comparisons are evaluated in succession, where the more complex model is selected if the computed $\Delta \mathrm{AIC}$ is greater than some pre-specified threshold, $\Delta \mathrm{AIC}_{\text {crit }}$. This process continues until either the most complex model is accepted or the improvement in the AIC score is too small to exceed the $\Delta A I C_{\text {crit }}$ threshold.

Figure 2. Type I error rates for the three MEDUSA algorithms. The three panels in each row summarize results for one algorithm (oMEDUSA, tMEDUSA, and nMEDUSA, from top to bottom), the three panels in each column summarize results for one tree size (with $N=100,1,000,10,000$ species, from left to right). Within each panel, we plot the number of terminal lineages, $k$, against the relative-extinction rate used in the simulation. Trees were simulated under a constant-rate birth-death process using the parameters summarized in Table S.2. The cells within each panel are colored as a heat map reflecting the frequency with which spurious diversification-rate shifts were inferred (see legend). The average Type I error rate is summarized in the lower right of each panel.

FIgURE 3. Critical and computed $\triangle$ AIC surfaces for the three MEDUSA algorithms. The four panels in each row correspond to one algorithm (oMEDUSA, tMEDUSA, and nMEDUSA, from top to bottom). The first column depicts the surface of $\Delta \mathrm{AIC}_{\text {crit }}$ threshold values for each algorithm; when the difference between the AIC scores of two competing models exceeds this threshold, the more complex model is selected. Columns $2-4$ summarize the $\Delta \mathrm{AIC}_{\text {crit }}$ values computed from simulated trees of each size $(N=100,1,000,10,000)$. The computed $\Delta$ AIC surfaces are colored to reflect the degree to which they exceed the corresponding $\Delta \mathrm{AIC}_{\text {crit }}$ threshold (see legend). Surfaces are computed from the trees used in the initial constant-rate simulation (c.f., Figure 2 ) under parameters described in Table S.2. Note the difference in the scale of $\triangle \mathrm{AIC}_{\text {crit }}$ values for the oMEDUSA algorithm. 
For detailed values, see Table S.6.

Figure 4. Type I error rates for completely sampled trees. Each row summarizes results for one algorithm (tMEDUSA and nMEDUSA on the top and bottom, respectively). Within each panel, we plot the number of species in the tree, $N$, against the relative-extinction rate used in the simulation. Trees were simulated under a constant-rate birth-death process using the parameters summarized in Table S.7. The cells within each panel are colored as a heat map reflecting the frequency with which spurious diversification-rate shifts were inferred (see FPR legend). The average Type I error rate is summarized in the lower right of each panel.

FiguRE 5. Model overfitting for terminal unresolved lineages. Trees of size $N$ ranging from 1 to 1000 were simulated under a constant-rate birth-death process using parameters summarized in Table S.8. The stem age of each tree was then used to estimate parameters of a constant-rate birth-death process using equation 2. We plot the difference between the likelihood under the true parameters and the maximum-likelihood estimates of the parameters, $\Delta \ln \mathcal{L}$, against the size of the tree, $N$ (truncated to 10 for visibility) and the relative extinction rate, $\epsilon$. Smaller values of $\Delta \ln \mathcal{L}$ indicate a stronger preference for the maximum-likelihood parameters against the true parameters.

\section{Figure 6. Bias in net-diversification rate estimates when the constant-rate model is} selected. The three panels in each row summarize results for one algorithm (oMEDUSA, tMEDUSA, and nMEDUSA, from top to bottom), the three panels in each column summarize results for one tree size (with $N=100,1,000,10,000$ species, from left to right). Within each panel, we plot the number of terminal lineages, $k$, against the relative-extinction rate used in the simulation. Trees were simulated under a constant-rate birth-death process using the parameters summarized in Table S.11. The cells within each panel are colored as a heat map reflecting the average difference between the estimated and true net-diversification rates when the one-rate model is correctly identified (see Bias legend). The average bias in the inferred net-diversification rate is summarized in the lower right of each panel.

FIgURE 7. Magnitude of shifts in net-diversification rate when a two-rate model is chosen. The three panels in each row summarize results for one algorithm (oMEDUSA, tMEDUSA, and nMEDUSA, from top to bottom), the three panels in each column summarize results for one tree size (with $N=100,1,000,10,000$ species, from left to right). Within each panel, we plot the 
number of terminal lineages, $k$, against the relative-extinction rate used in the simulation. Trees were simulated under a constant-rate birth-death process using the parameters summarized in Table S.15. The cells within each panel are colored as a heat map reflecting the average magnitude of shifts in net-diversification rate when a two-rate model is incorrectly identified (see Shift Magnitude legend). In order to accommodate the bimodal distribution of rate shifts, the magnitude of each netdiversification-rate shift was computed as the ratio of the higher rate to the lower rate, regardless of which rate corresponded to the background-rate category. The average magnitude of inferred diversification-rate shifts is summarized in the lower right of each panel. 


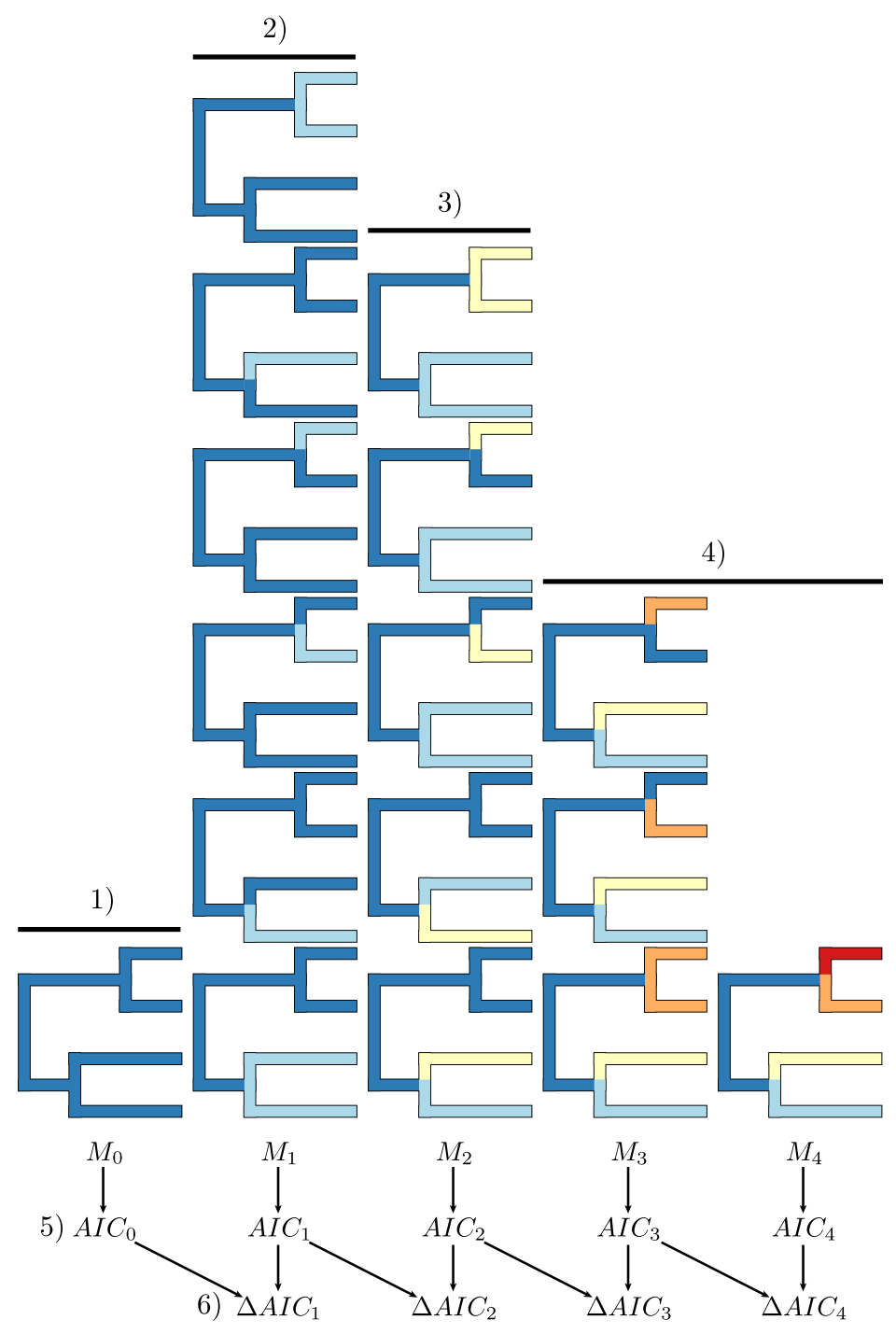

Figure 1: The MEDUSA algorithm. 1) The one-rate model, $M_{1}$, is fit to the data using maximum likelihood. 2) Every possible two-rate model is then fit to the data, and the models are ranked by their likelihood score (with the best model, $M_{2}$, at the bottom). 3) Every possible three-rate model that is consistent with the best two-rate model is then fit to the data, and the models are then ranked as in the previous step. 4) Step 3 is repeated for increasingly complex models until the most complex model has been evaluated. 5) The AIC score is computed for the best model in each index (i.e., with $0,1, \ldots$ rate shifts). 6) The $\Delta \mathrm{AIC}$ value is calculated for each pair of adjacent models (as the difference in their AIC scores). Each of the $\Delta A I C_{i}$ comparisons are evaluated in succession, where the more complex model is selected if the computed $\Delta \mathrm{AIC}$ is greater than some pre-specified threshold, $\Delta \mathrm{AIC}_{\text {crit }}$. This process continues until either the most complex model is accepted or the improvement in the AIC score is too small to exceed the $\Delta A I C_{\text {crit }}$ threshold. 

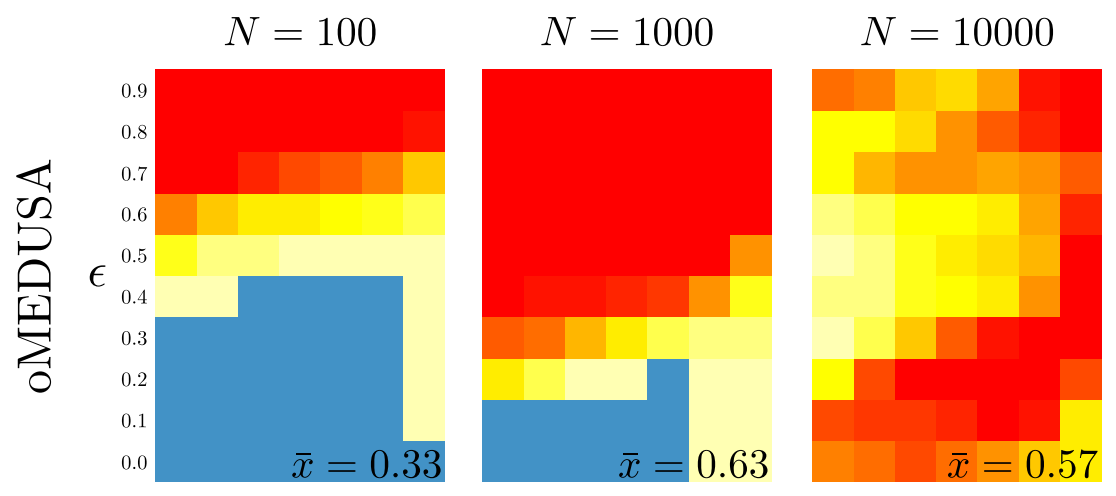

\section{FPR}
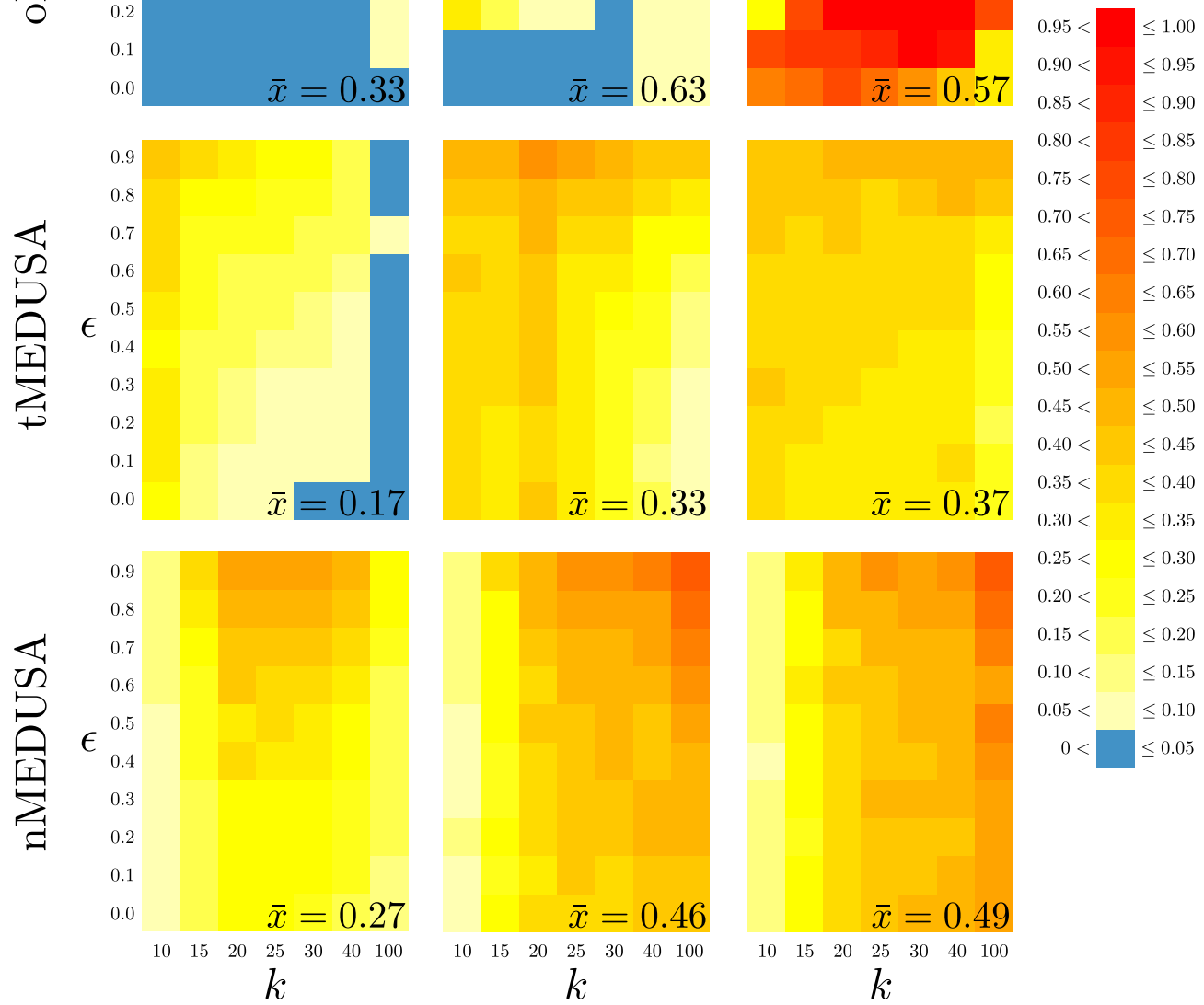

$0.80<\quad \leq 0.85$

$0.75<$

$0.70<$

$0.65<\quad \leq 0.70$

$0.60<\quad \leq 0.65$

$0.55<\quad \leq 0.60$

$0.50<\quad \leq 0.55$

$0.45<\quad \leq 0.50$

$0.40<\quad \leq 0.45$

$0.35<\quad \leq 0.40$

$0.30<\quad \leq 0.35$

$0.25<\quad \leq 0.30$

$0.20<\quad \leq 0.25$

$0.15<\quad \leq 0.20$

$0.10<\quad \leq 0.15$

$0.05<\quad \leq 0.10$

$0<\quad \leq 0.05$

FIgURE 2: Type I error rates for the three MEDUSA algorithms. The three panels in each row summarize results for one algorithm (oMEDUSA, tMEDUSA, and nMEDUSA, from top to bottom), the three panels in each column summarize results for one tree size (with $N=100,1,000,10,000$ species, from left to right). Within each panel, we plot the number of terminal lineages, $k$, against the relative-extinction rate used in the simulation. Trees were simulated under a constant-rate birth-death process using the parameters summarized in Table S.2. The cells within each panel are colored as a heat map reflecting the frequency with which spurious diversification-rate shifts were inferred (see legend). The average Type I error rate is summarized in the lower right of each panel. 

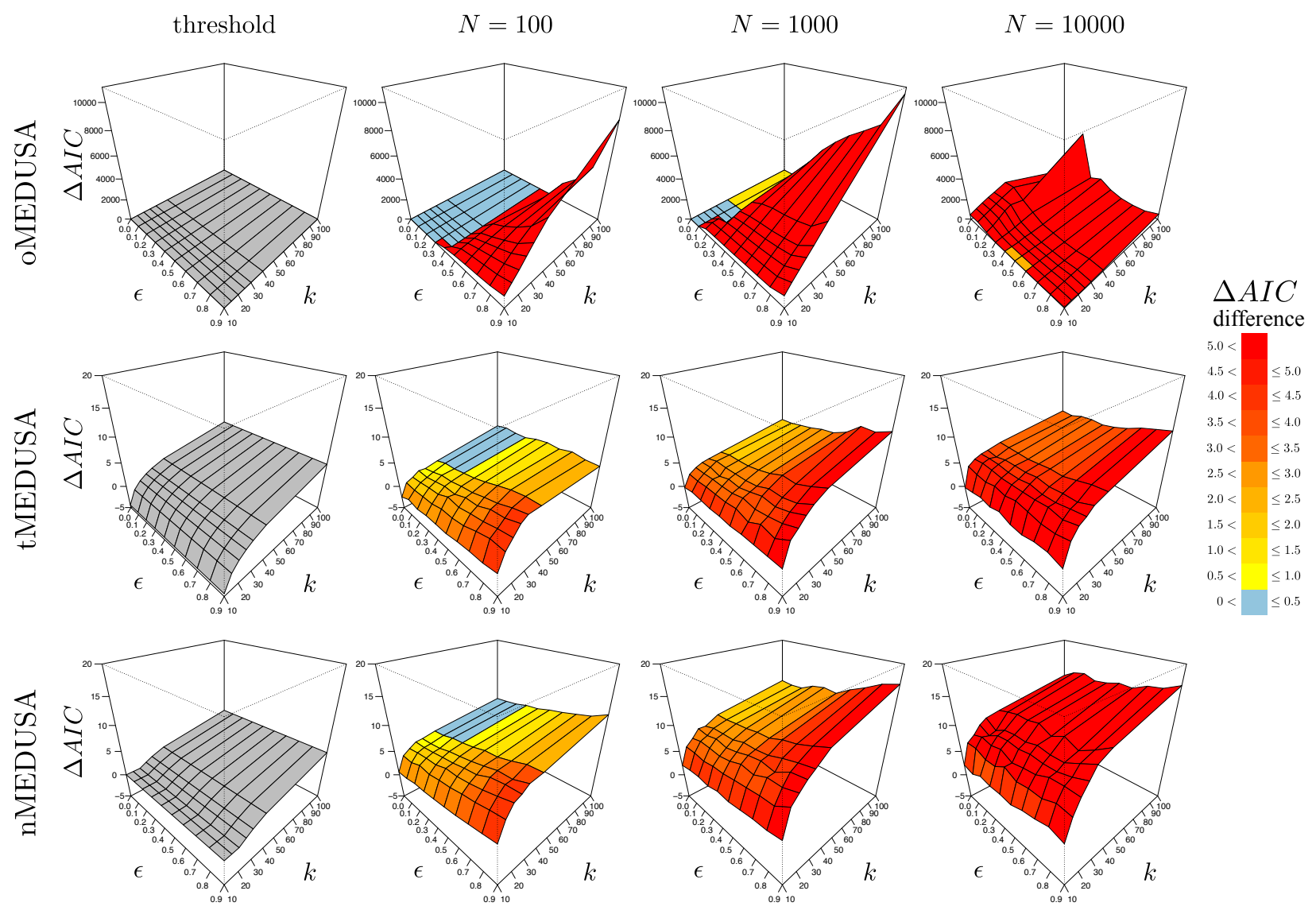

FIGURE 3: Critical and computed $\triangle \mathrm{AIC}$ surfaces for the three MEDUSA algorithms. The four panels in each row correspond to one algorithm (oMEDUSA, tMEDUSA, and nMEDUSA, from top to bottom). The first column depicts the surface of $\Delta \mathrm{AIC}_{\text {crit }}$ threshold values for each algorithm; when the difference between the AIC scores of two competing models exceeds this threshold, the more complex model is selected. Columns $2-4$ summarize the $\Delta \mathrm{AIC}_{\text {crit }}$ values computed from simulated trees of each size $(N=100,1,000,10,000)$. The computed $\Delta$ AIC surfaces are colored to reflect the degree to which they exceed the corresponding $\Delta \mathrm{AIC}_{\text {crit }}$ threshold (see legend). Surfaces are computed from the trees used in the initial constant-rate simulation (c.f., Figure 2) under parameters described in Table S.2. Note the difference in the scale of $\triangle \mathrm{AIC}_{\text {crit }}$ values for the oMEDUSA algorithm. For detailed values, see Table S.6 

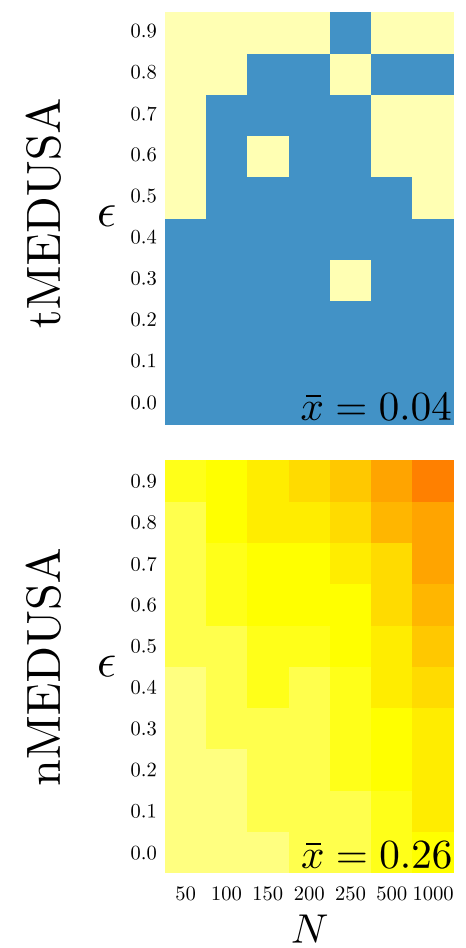

\section{FPR}

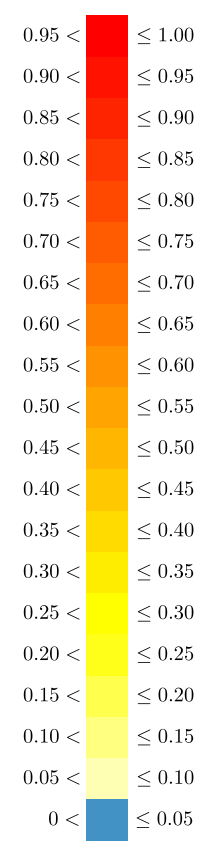

FIgURE 4: Type I error rates for completely sampled trees. Each row summarizes results for one algorithm (tMEDUSA and nMEDUSA on the top and bottom, respectively). Within each panel, we plot the number of species in the tree, $N$, against the relative-extinction rate used in the simulation. Trees were simulated under a constant-rate birth-death process using the parameters summarized in Table S.7. The cells within each panel are colored as a heat map reflecting the frequency with which spurious diversification-rate shifts were inferred (see FPR legend). The average Type I error rate is summarized in the lower right of each panel. 


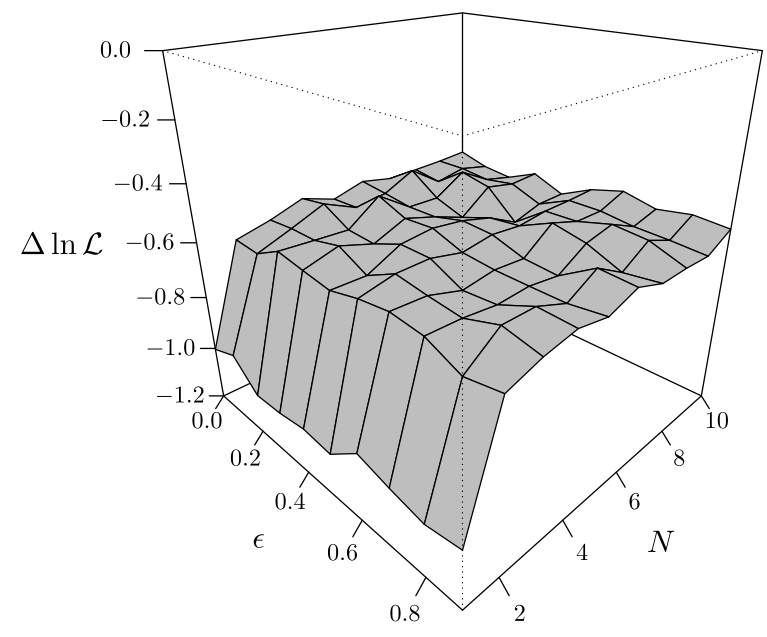

Figure 5: Model overfitting for terminal unresolved lineages. Trees of size $N$ ranging from 1 to 1000 were simulated under a constant-rate birth-death process using parameters summarized in Table S.8. The stem age of each tree was then used to estimate parameters of a constant-rate birth-death process using equation 2. We plot the difference between the likelihood under the true parameters and the maximum-likelihood estimates of the parameters, $\Delta \ln \mathcal{L}$, against the size of the tree, $N$ (truncated to 10 for visibility) and the relative extinction rate, $\epsilon$. Smaller values of $\Delta \ln \mathcal{L}$ indicate a stronger preference for the maximum-likelihood parameters against the true parameters. 

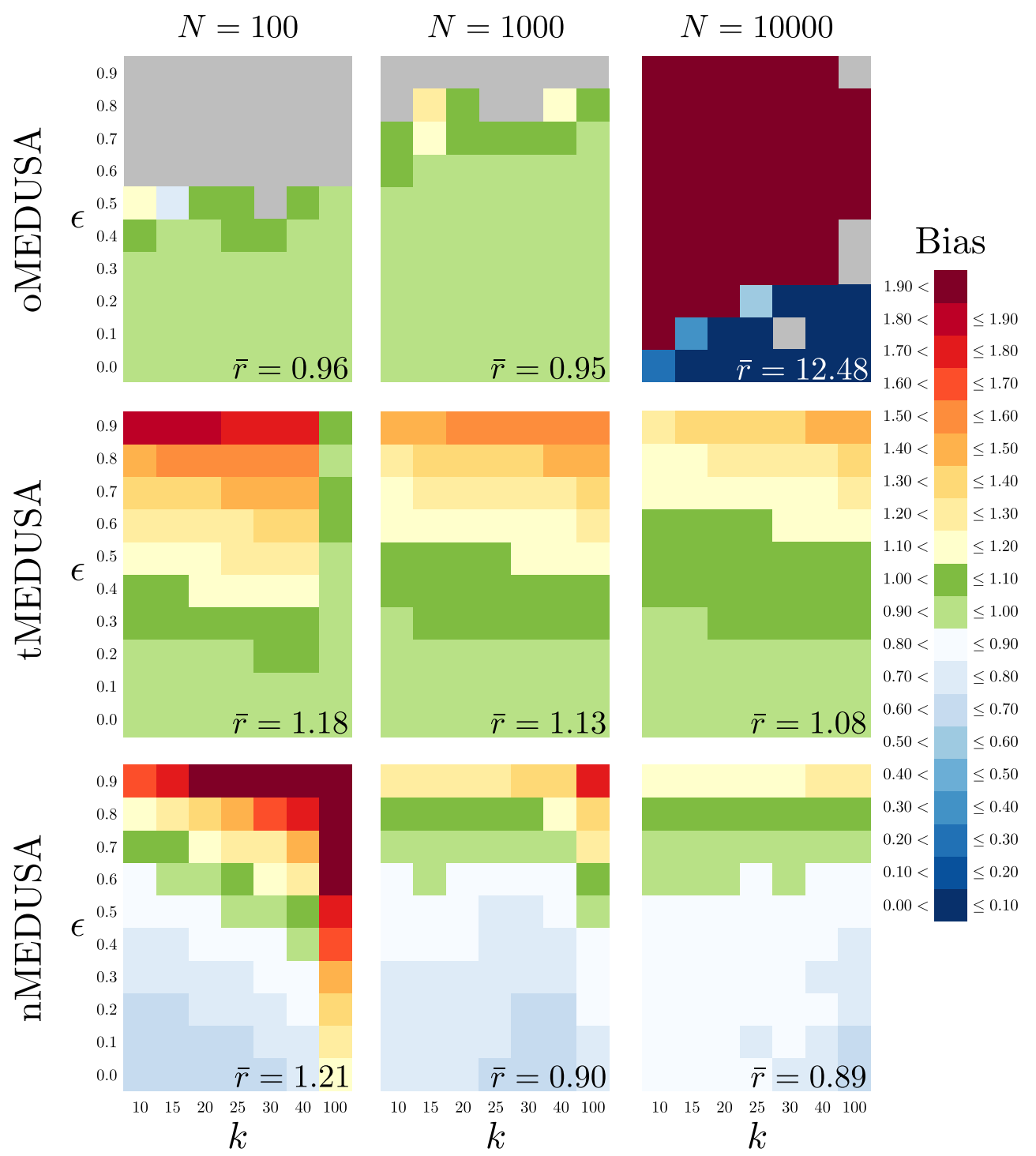

$1.50<\quad \leq 1.60$

$1.40<\quad \leq 1.50$

$1.30<\quad \leq 1.40$

$1.20<\quad \leq 1.30$

$1.10<\quad \leq 1.20$

$1.00<\quad \leq 1.10$

$0.90<\quad \leq 1.00$

$0.80<\quad \leq 0.90$

$0.70<\quad \leq 0.80$

$\bar{r}=1.13$
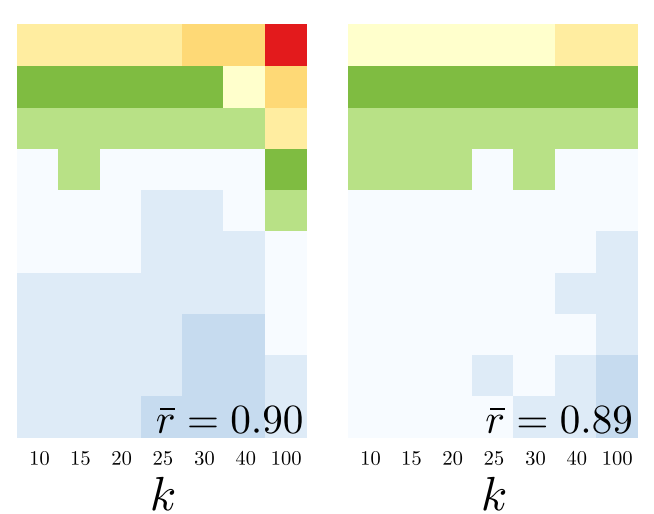

$0.60<\quad \leq 0.70$

$0.50<\quad \leq 0.60$

$0.40<\quad \leq 0.50$

$0.30<\quad \leq 0.40$

$0.20<\quad \leq 0.30$

$0.10<$

$0.00<\quad \leq 0.10$

FIGURE 6: Bias in net-diversification rate estimates when the constant-rate model is selected. The three panels in each row summarize results for one algorithm (oMEDUSA, tMEDUSA, and nMEDUSA, from top to bottom), the three panels in each column summarize results for one tree size (with $N=100,1,000,10,000$ species, from left to right). Within each panel, we plot the number of terminal lineages, $k$, against the relative-extinction rate used in the simulation. Trees were simulated under a constant-rate birth-death process using the parameters summarized in Table S.11. The cells within each panel are colored as a heat map reflecting the average difference between the estimated and true net-diversification rates when the one-rate model is correctly identified (see Bias legend). The average bias in the inferred net-diversification rate is summarized in the lower right of each panel. 

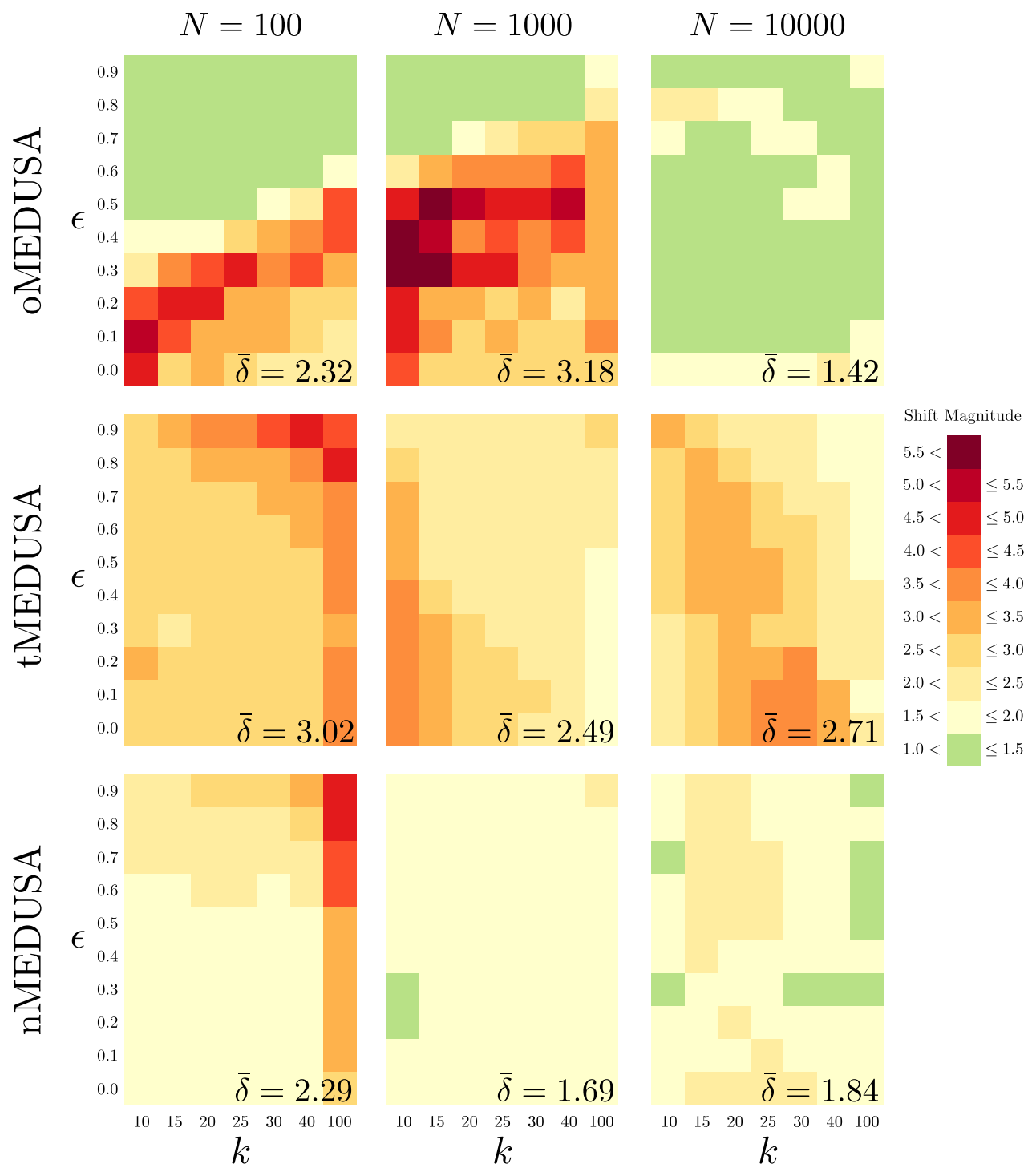

Figure 7: Magnitude of shifts in net-diversification rate when a two-rate model is chosen. The three panels in each row summarize results for one algorithm (oMEDUSA, tMEDUSA, and nMEDUSA, from top to bottom), the three panels in each column summarize results for one tree size (with $N=100,1,000,10,000$ species, from left to right). Within each panel, we plot the number of terminal lineages, $k$, against the relative-extinction rate used in the simulation. Trees were simulated under a constant-rate birth-death process using the parameters summarized in Table S.15. The cells within each panel are colored as a heat map reflecting the average magnitude of shifts in net-diversification rate when a two-rate model is incorrectly identified (see Shift Magnitude legend). In order to accommodate the bimodal distribution of rate shifts, the magnitude of each netdiversification-rate shift was computed as the ratio of the higher rate to the lower rate, regardless of which rate corresponded to the background-rate category. The average magnitude of inferred diversification-rate shifts is summarized in the lower right of each panel. 


\section{SUPPLEMENTARY MATERIAL}

\section{Contents}

S.1 A MORE DETAILED DESCRIPTION OF THE MEDUSA ALGORITHM $4 \mathbf{4 1}$

$\begin{array}{lll}\text { S.2 } & \text { SURVEY OF EMPIRICAL STUDIES USING MEDUSA } & 42\end{array}$

S.3 The EFFECT OF RELATIVE-EXTINCTION RATE, TREE SiZE, AND RESOLUTiON (STEM

SHIFTS

S.4 EXPLORING THE IMPACT OF MONTE CARLO ERROR ON TYPE I ERROR RATES (STEM

SHIFTS

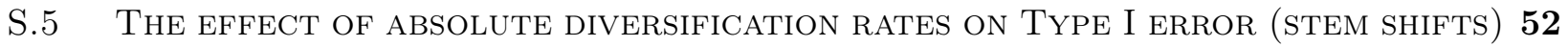

S.6 EXPLORING THRESHOLD EFFECTS ON TYPE I ERROR RATES (STEM SHIFTS) $\quad \mathbf{5 7}$

S.7 $\quad$ EXPLORING TYPE I ERROR RATES FOR COMPLETE SPECIES TREES (STEM SHIFTS) 59

S.8 ASSESSING THE IMPACT OF TERMINAL UNRESOLVED CLADES (STEM SHIFTS) $\quad 60$

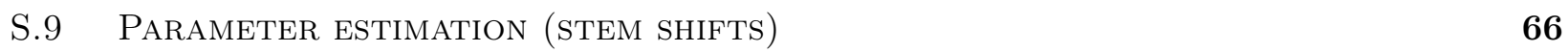

S.10 ThE EFFECT OF RELATIVE-EXTINCTION RATE, TREE SIZE, AND PHYLOGENETIC RES-

$\begin{array}{lr}\text { OLUTION (CROWN SHIFTS). } & \mathbf{8 0}\end{array}$

S.11 EXPlORING THE IMPACT OF MONTE CARLO ERROR ON TYPE I ERROR RATES

$\begin{array}{ll}\text { (CROWN SHIFTS) } & 82\end{array}$

S.12 The EFFECT OF ABSOLUte DIVERSIFICATION RATES ON TyPE I ERROR (CROWN

$\begin{array}{llr}\text { SHIFTS } & 84\end{array}$

\begin{tabular}{lll}
\hline S.13 EXPlORING THRESHOLD EFFECTS ON TYPE I ERROR RATES (CROWN SHIFTS) & $\mathbf{8 8}$
\end{tabular}

S.14 EXPlORING TyPE I ERROR RATES FOR COMPLETE SPECIES TREES (CROWN SHIFTS) 90 
bioRxiv preprint doi: https://doi.org/10.1101/011452; this version posted November 15,2014 . The copyright holder for this preprint (which was not certified by peer review) is the author/funder, who has granted bioRxiv a license to display the preprint in perpetuity. It is made available under aCC-BY-ND 4.0 International license.

S.15 PARAMETER ESTIMATION (CROWN SHIFTS)

SUPPLEMENTARY REFERENCES

108 


\section{S.1 A More Detalled Description of the medusa Algorithm}

There are $(2 k-2)$ clades in the tree, $k$ of which are terminal branches and $(k-2)$ of which are nodes. Label the terminal branches in the tree 1 through $k$, and all the non-root nodes in the tree $(k+1)$ through $(2 k-2)$ in arbitrary order.

1. Fit a single-rate diversification model to $\Psi$ by maximum likelihood using equation (3); compute $A I C_{\text {one-rate }}$

2. Set $i=2$. This is an index of the number of rate classes under consideration.

3. Set $j=1$. This is an index of the clade under consideration for a rate shift.

4. Visit clade $j$.

(a) If clade $j$ already has a rate shift, go to step 5.

(b) Define a partition of branches into categories where the descendant branches of clade $j$ are in one rate category, all the descendant branches of any other rate shifts are in their own rate categories, and the rest of the branches are in a "background" rate category. When a branch might belong to multiple rate categories, the branch is placed in the category defined by the youngest clade.

(c) Fit a multiple-rate diversification model defined by the partition to $\Psi$ by maximum likelihood using equation (3); compute $A I C_{i \text {-rate }}^{j}$.

5. If $j=(2 k-2)$, go to step 8 .

6. Set $j=j+1$.

7. Return to step 4 .

8. Determine the best (lowest) $A I C_{i \text {-rate }}^{j}$ Call it $A I C_{i \text {-rate }}$.

9. Compute $\triangle A I C=A I C_{(i-1) \text {-rate }}-A I C_{i \text {-rate }}$.

10. If $\triangle A I C \geq \triangle A I C_{\text {crit }}$, accept the shift at the clade with the best $A I C_{i \text {-rate }}^{j}$ Otherwise, go to step 14 .

11. If $i=(2 k-2)$, go to step 14 .

12. Set $i=i+1$.

13. Return to step 3.

14. Terminate the algorithm. 


\section{S.2 Survey of EMPIRICAL STUdies USING MEDUSA}

\begin{tabular}{|c|c|c|c|c|c|c|c|}
\hline Study group & $\#$ species $(N)$ & \# terminals $(k)$ & $\lambda_{0}$ & $\mu_{0}$ & \# shifts & Algorithm & Study \\
\hline Bromeliaceae & 3140 & 26 & & & 3 & tMEDUSA & Givnish et al. (2014) \\
\hline Cephalotes & 118 & 19 & 0.074 & 0.000 & 1 & tMEDUSA $^{\dagger}$ & Price et al. (2014) \\
\hline Allocentroleniae & 165 & 47 & 0.144 & 0.000 & 2 & tMEDUSA & Castroviejo-Fisher et al. (2014) \\
\hline Cyperaceae & 5477 & 28 & 0.131 & 0.072 & 3 & tMEDUSA & Escudero and Hipp (2013) \\
\hline Lepidoptera & 170000 & 116 & & & 4 & $\mathrm{tMEDUSA}^{\dagger}$ & Wahlberg et al. (2013) \\
\hline Conifers & 489 & 489 & & & 5 & tMEDUSA & Leslie et al. (2013) \\
\hline Legumes & 19500 & 331 & 0.418 & 0.353 & 10 & tMEDUSA $^{\dagger}$ & Koenen et al. (2013) \\
\hline Campanulidae & 8911 & 121 & & & 6 & $\mathrm{tMEDUSA}^{\dagger}$ & Beaulieu and Donoghue (2013) \\
\hline Muroid rodents & 1638 & 221 & & & 8 & $\mathrm{tMEDUSA}^{\dagger}$ & Schenk et al. (2013) \\
\hline Apidae & 1628 & 18 & & & 1 & tMEDUSA & Litman et al. (2013) \\
\hline Balistoidea & 143 & 30 & & & 1 & $\mathrm{tMEDUSA}^{\dagger}$ & Santini et al. $(2013 \mathrm{c})$ \\
\hline Amoebozoa & 2691 & 73 & & & 6 & $\mathrm{tMEDUSA}^{\dagger}$ & Fiz-Palacios et al. (2013) \\
\hline Halictine bees & & & & & 0 & $\mathrm{tMEDUSA}^{\dagger}$ & Groom et al. (2013) \\
\hline Cichlids & 1638 & 18 & 0.117 & 0.048 & 1 & $\mathrm{tMEDUSA}^{\dagger}$ & McMahan et al. (2013) \\
\hline Ants & 12199 & 295 & 0.684 & 0.645 & 10 & oMEDUSA $^{\dagger}$ & Moreau and Bell (2013) \\
\hline Metschnikowia & 82 & 82 & 0.035 & 0.000 & 2 & oMEDUSA & Guzman et al. (2013) \\
\hline Spiny-rayed fishes & 19277 & 181 & & & 9 & $\mathrm{tMEDUSA}^{\dagger}$ & Near et al. (2013) \\
\hline Scombridae & 47 & 47 & & & 2 & $\mathrm{tMEDUSA}^{\dagger}$ & Santini et al. (2013a) \\
\hline Eucharitidae & & 68 & & & 2 & tMEDUSA & Murray et al. 2013) \\
\hline Tetradontidae & 178 & 18 & 0.131 & 0.005 & 1 & tMEDUSA & Santini et al. (2013b) \\
\hline Cymothoe & 82 & 82 & & & 1 & $\mathrm{tMEDUSA}^{\dagger}$ & Van Velzen et al. (2013) \\
\hline New World oscines & 204 & 17 & 0.220 & 0.000 & 2 & tMEDUSA & Barker et al. (2013) \\
\hline Arecaceae & 2355 & 183 & 0.050 & 0.000 & 13 & tMEDUSA & Baker and Couvreur (2013) \\
\hline Cyanobacteria & 1220 & 32 & & & 2 & oMEDUSA & Schirrmeister et al. (2013) \\
\hline Pomacentridae & 386 & 208 & & & 1 & $\mathrm{tMEDUSA}^{\dagger}$ & Frederich et al. $(2013)$ \\
\hline Scutigeromorph centipedes & 95 & 48 & & & 1 & nMEDUSA & Giribet and Edgecombe (2013) \\
\hline Bactridinae & 166 & 23 & 0.089 & 0.000 & 1 & tMEDUSA & Roncal et al. (2013) \\
\hline Iguania & 1664 & 19 & 0.050 & 0.000 & 2 & $\mathrm{tMEDUSA}^{\dagger}$ & Blankers et al. (2013) \\
\hline Aves & 9993 & 9993 & & & 25 & tMEDUSA & Jetz et al. $(2012)$ \\
\hline Drosophila & 1506 & 13 & & & 1 & $\mathrm{oMEDUSA}^{\dagger}$ & Morales-Hojas and Vieira (2012) \\
\hline Malphigiales & 16043 & 41 & & & 6 & tMEDUSA & Xi et al. (2012) \\
\hline Angiosperms & 268301 & 330 & & & 39 & $\mathrm{tMEDUSA}^{\dagger}$ & Rabosky et al. (2012) \\
\hline Gymnosperms & 2837 & 12 & & & 1 & $\mathrm{tMEDUSA}^{\dagger}$ & Rabosky et al. 2012 \\
\hline Ferns & 9118 & 21 & & & 3 & $\mathrm{tMEDUSA}^{\dagger}$ & Rabosky et al. (2012) \\
\hline Chondrichthyes & 991 & 57 & & & 4 & $\mathrm{tMEDUSA}^{\dagger}$ & Rabosky et al. (2012) \\
\hline Actinopterygii & 18613 & 16 & & & 1 & $\mathrm{tMEDUSA}^{\dagger}$ & Rabosky et al. (2012) \\
\hline Amphibia & 6378 & 74 & & & 8 & $\mathrm{tMEDUSA}^{\dagger}$ & Rabosky et al. 2012 \\
\hline Mammalia & 5279 & 149 & & & 11 & $\mathrm{tMEDUSA}^{\dagger}$ & Rabosky et al. (2012) \\
\hline Aves & 10237 & 163 & & & 15 & $\mathrm{tMEDUSA}^{\dagger}$ & Rabosky et al. (2012) \\
\hline
\end{tabular}




\begin{tabular}{|c|c|c|c|c|c|c|c|}
\hline Squamata & 6979 & 53 & & & 5 & tMEDUSA $^{\dagger}$ & Rabosky et al. (2012) \\
\hline Araneae & 8776 & 24 & & & 3 & $\mathrm{tMEDUSA}^{\dagger}$ & Rabosky et al. (2012) \\
\hline Coleoptera & 342201 & 183 & & & 17 & $\mathrm{tMEDUSA}^{\dagger}$ & Rabosky et al. (2012) \\
\hline Diptera & 87899 & 61 & & & 9 & tMEDUSA $^{\dagger}$ & Rabosky et al. (2012) \\
\hline Coprinellus mushrooms & 304 & 242 & & & 1 & $\mathrm{oMEDUSA}^{\dagger}$ & Nagy et al. (2012) \\
\hline Carex & 2015 & 4 & 0.879 & 0.866 & 1 & $\mathrm{oMEDUSA}^{\dagger}$ & Escudero et al. (2012) \\
\hline Tetrapontidae & 37 & 25 & 3.950 & 0.000 & 1 & oMEDUSA & Davis et al. (2012) \\
\hline Amanita & 99 & 99 & & & 0 & oMEDUSA $^{\dagger}$ & Ryberg and Matheny (2012) \\
\hline Cortinarius & 796 & 796 & 0.110 & 0.000 & 2 & $\mathrm{oMEDUSA}^{\dagger}$ & Ryberg and Matheny (2012) \\
\hline Descolea & 27 & 27 & & & 0 & oMEDUSA $^{\dagger}$ & Ryberg and Matheny (2012) \\
\hline Hygrophorus & 21 & 21 & & & 0 & $\mathrm{oMEDUSA}^{\dagger}$ & Ryberg and Matheny (2012) \\
\hline Hebelomateae & 117 & 117 & 0.110 & 0.000 & 1 & oMEDUSA $^{\dagger}$ & Ryberg and Matheny 2012) \\
\hline Hydnangiaceae & 37 & 37 & & & 0 & oMEDUSA $^{\dagger}$ & Ryberg and Matheny (2012) \\
\hline Tricholoma & 78 & 78 & & & 0 & $\mathrm{oMEDUSA}^{\dagger}$ & Ryberg and Matheny (2012) \\
\hline Inocybaceae & 489 & 489 & & & 0 & $\mathrm{oMEDUSA}^{\dagger}$ & Ryberg and Matheny (2012) \\
\hline Phaeocollybia & 23 & 23 & 0.020 & 0.000 & 1 & oMEDUSA $^{\dagger}$ & Ryberg and Matheny (2012) \\
\hline Lupinus & 275 & 19 & & & 3 & oMEDUSA & Drummond et al. (2012) \\
\hline Furnariidae & 293 & 293 & & & 3 & $\mathrm{oMEDUSA}^{\dagger}$ & Claramunt et al. (2012) \\
\hline Lepidoptera & 18187 & 28 & & & 3 & $\mathrm{oMEDUSA}^{\dagger}$ & Heikkila et al. (2012) \\
\hline Notothenioids & 122 & 83 & & & 2 & $\mathrm{oMEDUSA}^{\dagger}$ & Near et al. $(2012)$ \\
\hline Mammalia & 5358 & 149 & 0.050 & 0.025 & 9 & oMEDUSA $^{\dagger}$ & Yu et al. (2012) \\
\hline Snakes & 3256 & 40 & 0.082 & 0.051 & 4 & oMEDUSA $^{\dagger}$ & Pyron and Burbrink (2012) \\
\hline Megachilidae & 98 & 82 & & & 2 & $\mathrm{oMEDUSA}^{\dagger}$ & Litman et al. (2011) \\
\hline Labridae & 630 & 46 & 0.100 & 0.000 & 3 & oMEDUSA $^{\dagger}$ & Cowman and Bellwood 2011) \\
\hline Chaetodontidae & 131 & 15 & 0.100 & 0.000 & 1 & $\mathrm{oMEDUSA}^{\dagger}$ & Cowman and Bellwood (2011) \\
\hline Apogonidae & 289 & 16 & 0.093 & 0.000 & 2 & $\mathrm{oMEDUSA}^{\dagger}$ & Cowman and Bellwood 2011) \\
\hline Pomacentridae & 280 & 36 & 0.050 & 0.000 & 1 & oMEDUSA $^{\dagger}$ & Cowman and Bellwood (2011) \\
\hline Land plants & 706 & 706 & & & 69 & $\mathrm{oMEDUSA}^{\dagger}$ & Fiz-Palacios et al. $(2011)$ \\
\hline Onagraceae & 262 & 27 & & & 1 & oMEDUSA $^{\dagger}$ & Johnson et al. (2011) \\
\hline Parrots & 353 & 31 & 0.080 & 0.002 & 1 & $\mathrm{oMEDUSA}^{\dagger}$ & Schweizer et al. (2011) \\
\hline Furnariidae & 285 & 285 & 0.100 & 0.000 & 2 & $\mathrm{oMEDUSA}^{\dagger}$ & Derryberry et al. (2011) \\
\hline Hydyosmum & 76 & 7 & 0.064 & 0.050 & 1 & $\mathrm{oMEDUSA}^{\dagger}$ & Antonelli and Sanmartin (2011) \\
\hline Kladothrips & 41 & 17 & & & 0 & $\mathrm{oMEDUSA}^{\dagger}$ & McLeish (2011) \\
\hline Allodapine bees & 77 & 14 & 0.050 & 0.000 & 1 & oMEDUSA $^{\dagger}$ & Chenoweth and Schwarz (2011) \\
\hline Leiognathidae & 51 & 9 & & & 0 & $\mathrm{oMEDUSA}^{\dagger}$ & Chakrabarty et al. (2011) \\
\hline Cactaceae & 1892 & 132 & 4.454 & 4.022 & 8 & $\mathrm{oMEDUSA}^{\dagger}$ & Arakaki et al. (2011) \\
\hline Diptera & 145575 & 143 & 0.023 & 0.001 & 9 & $\mathrm{oMEDUSA}^{\dagger}$ & Matthews et al. (2011) \\
\hline Hyrdrophiini & 180 & 33 & & & 1 & $\mathrm{oMEDUSA}^{\dagger}$ & Wiegmann et al. (2011) \\
\hline Cetacea & 74 & 59 & & & 1 & $\mathrm{oMEDUSA}^{\dagger}$ & Slater et al. $(2010)$ \\
\hline Ray-finned fishes & 26685 & 29 & 0.011 & 0.000 & 4 & oMEDUSA $^{\dagger}$ & Santini et al. $(2009)$ \\
\hline \multirow[t]{2}{*}{ Gnathostomes } & 57501 & 47 & 1.000 & 0.990 & 9 & oMEDUSA $^{\dagger}$ & Alfaro et al. (2009) \\
\hline & 16863.641 & 232.076 & 0.434 & 0.230 & 4.938 & & \\
\hline
\end{tabular}


N.B. The total number of species $(N)$, the number of incompletely resolved terminal lineages $(k)$, the estimated background speciation $\left(\lambda_{0}\right)$ and extinction $\left(\mu_{0}\right)$ rates in species per species per million years, and the inferred number of diversification-rate shifts (Shifts) inferred by published studies using various MEDUSA algorithms. ${ }^{\dagger}$ : These studies did not provide sufficient information to unambiguously determine the particular algorithm used; in these cases, we assigned oMEDUSA if the study was published before the release of GEIGER 1.99 and tMEDUSA if it was published after. 


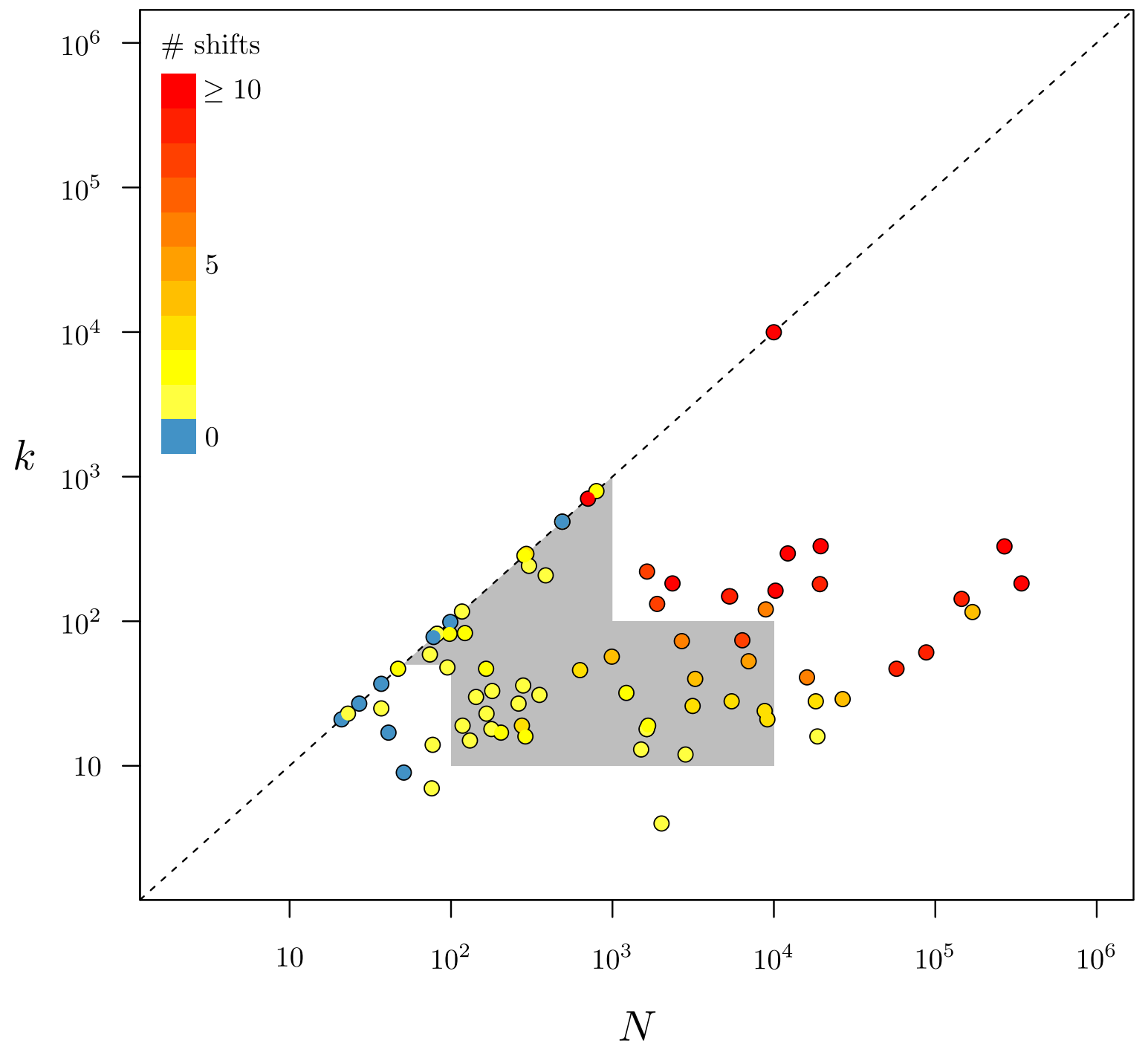

FiguRE S.1: Parameter space of empirical datasets that have been analyzed with MEDUSA: tree size and phylogenetic resolution. A log-log plot of the number of species in each dataset, $N$, against the number of terminal lineages in each tree, $k$. Each dataset is colored by the number of shifts inferred by MEDUSA. Datasets with complete species sampling fall on the dashed line, while those with incomplete sampling fall below the dashed line. The grey area contains the values of $N$ and $k$ used in our simulation study. 


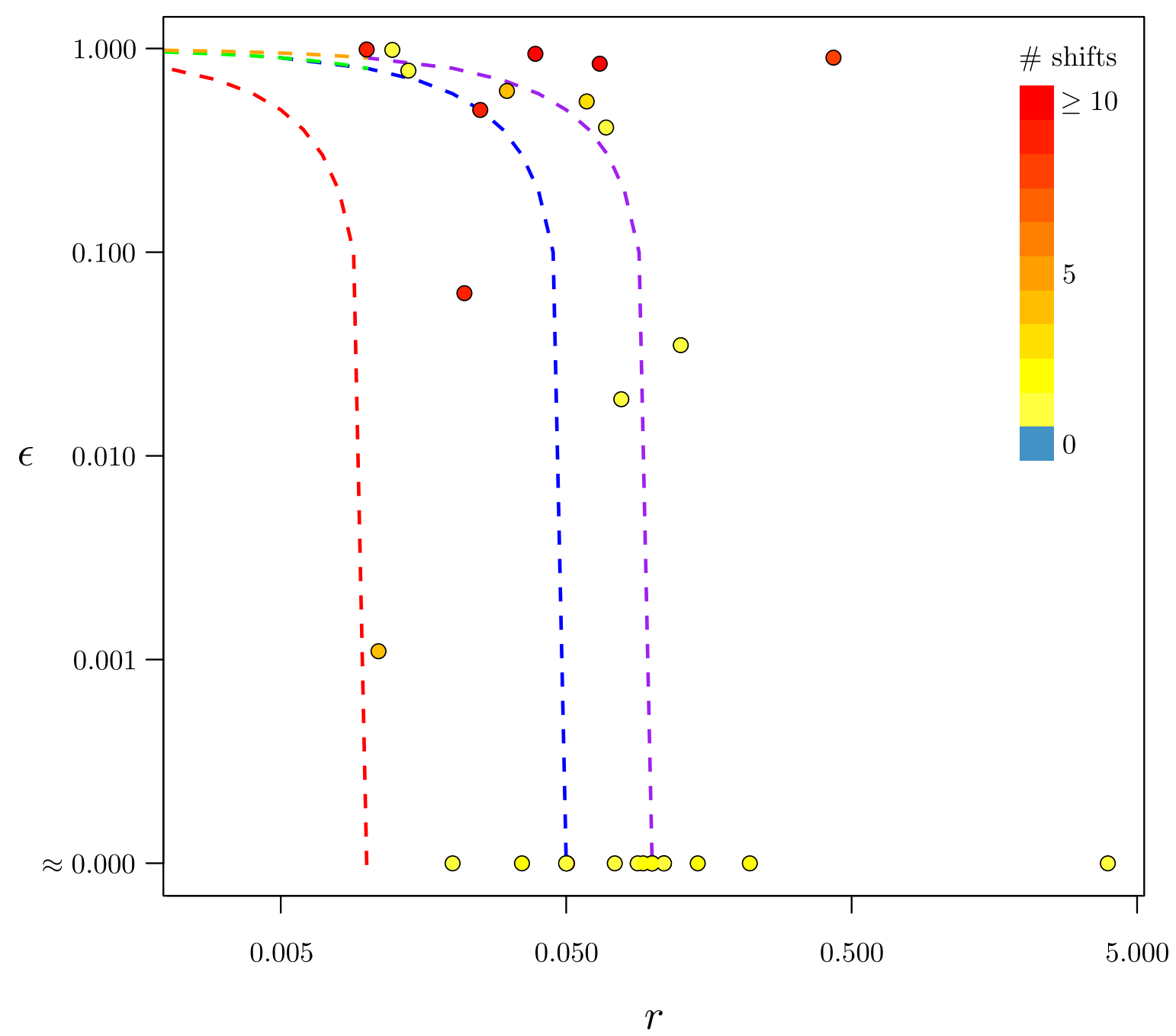

FIgURE S.2: Parameter space of empirical datasets that have been analyzed with MEDUSA: diversification rates. A log-log plot of the net-diversification rate, $r$, against the relativeextinction rate, $\epsilon$, for the background rate category of empirical datasets analyzed with MEDUSA. Each dataset is colored by the number of shifts inferred by MEDUSA. Dashed lines correspond to parameter settings used in our simulation study. Red: Table S.2. Blue: Table S.4, left; Purple: Table S.4. right; Green: Table S.5, left; Orange: Table S.5, right. 


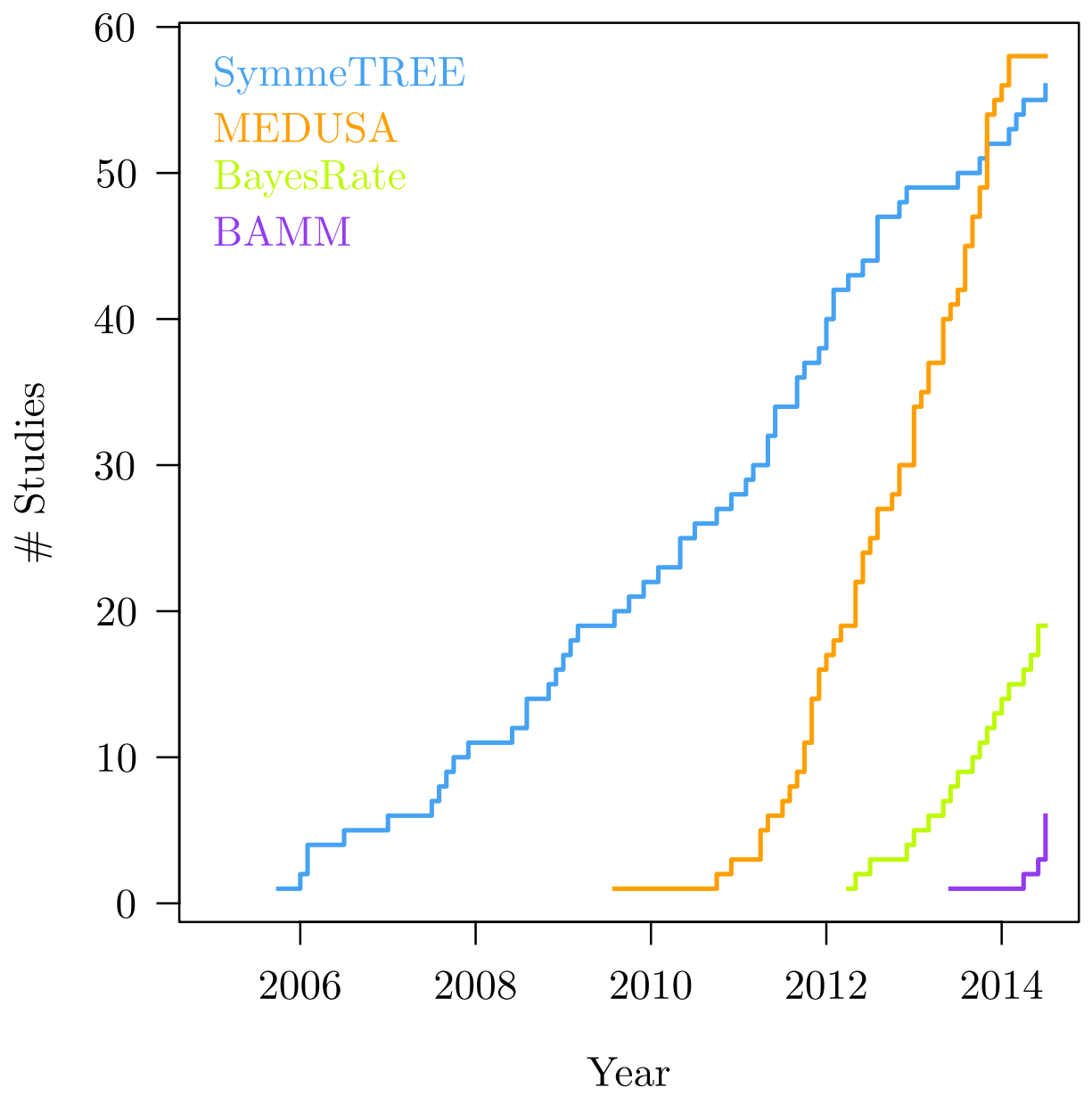

FIgURE S.3: Usage of alternative methods for detecting lineage-specific diversificationrates in empirical studies. 
bioRxiv preprint doi: https://doi.org/10.1101/011452; this version posted November 15,2014 . The copyright holder for this preprint (which was not certified by peer review) is the author/funder, who has granted bioRxiv a license to display the preprint in perpetuity. It is made available under aCC-BY-ND 4.0 International license. 
bioRxiv preprint doi: https://doi.org/10.1101/011452; this version posted November 15,2014 . The copyright holder for this preprint (which was not certified by peer review) is the author/funder, who has granted bioRxiv a license to display the preprint in perpetuity. It is made available under aCC-BY-ND 4.0 International license.

S.3 The effect of Relative-extinction Rate, tree size, And RESOlution (stem Shifts)

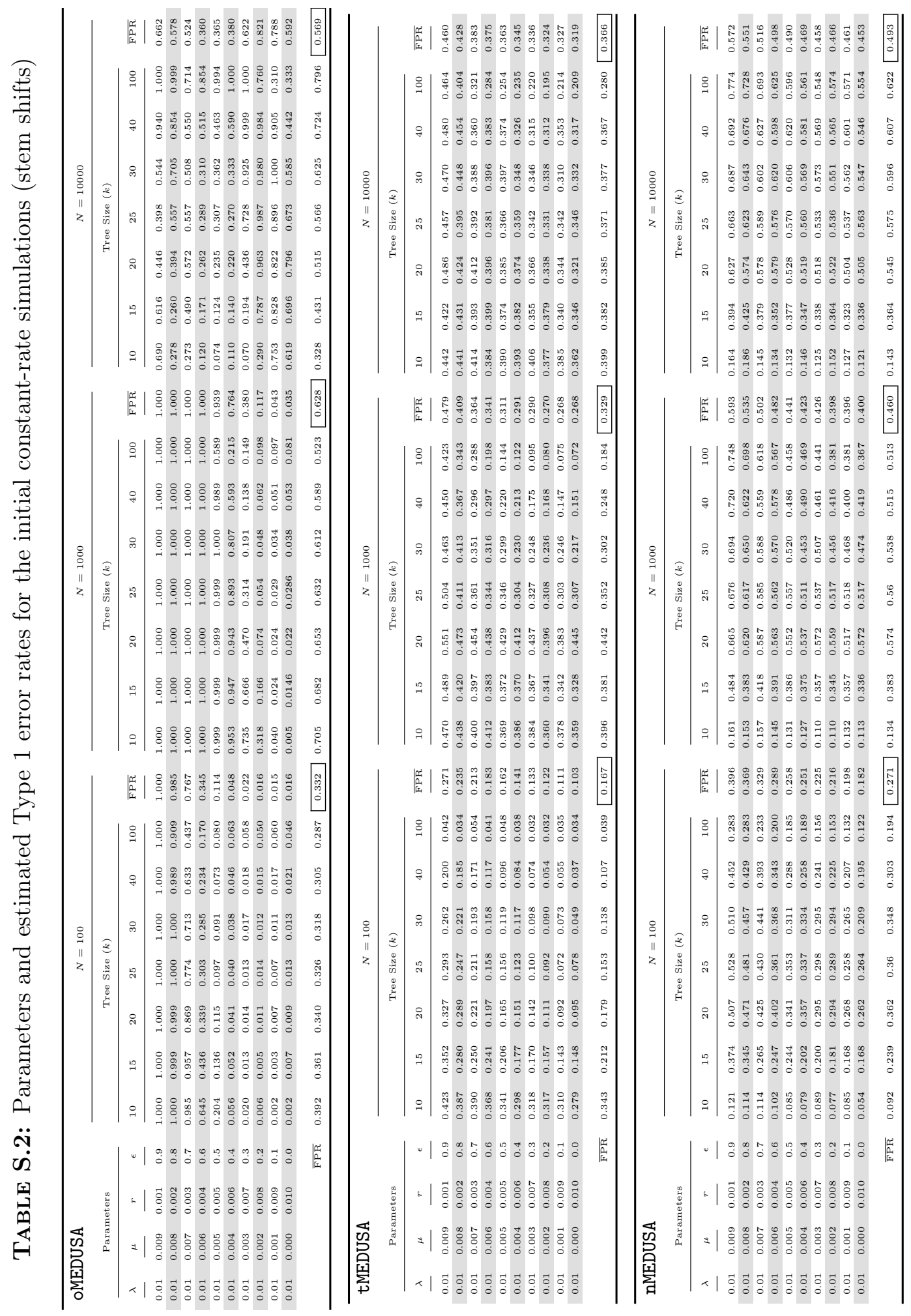




\section{S.4 Exploring the impact of Monte Carlo error on Type I error Rates (stem} SHIFTS)

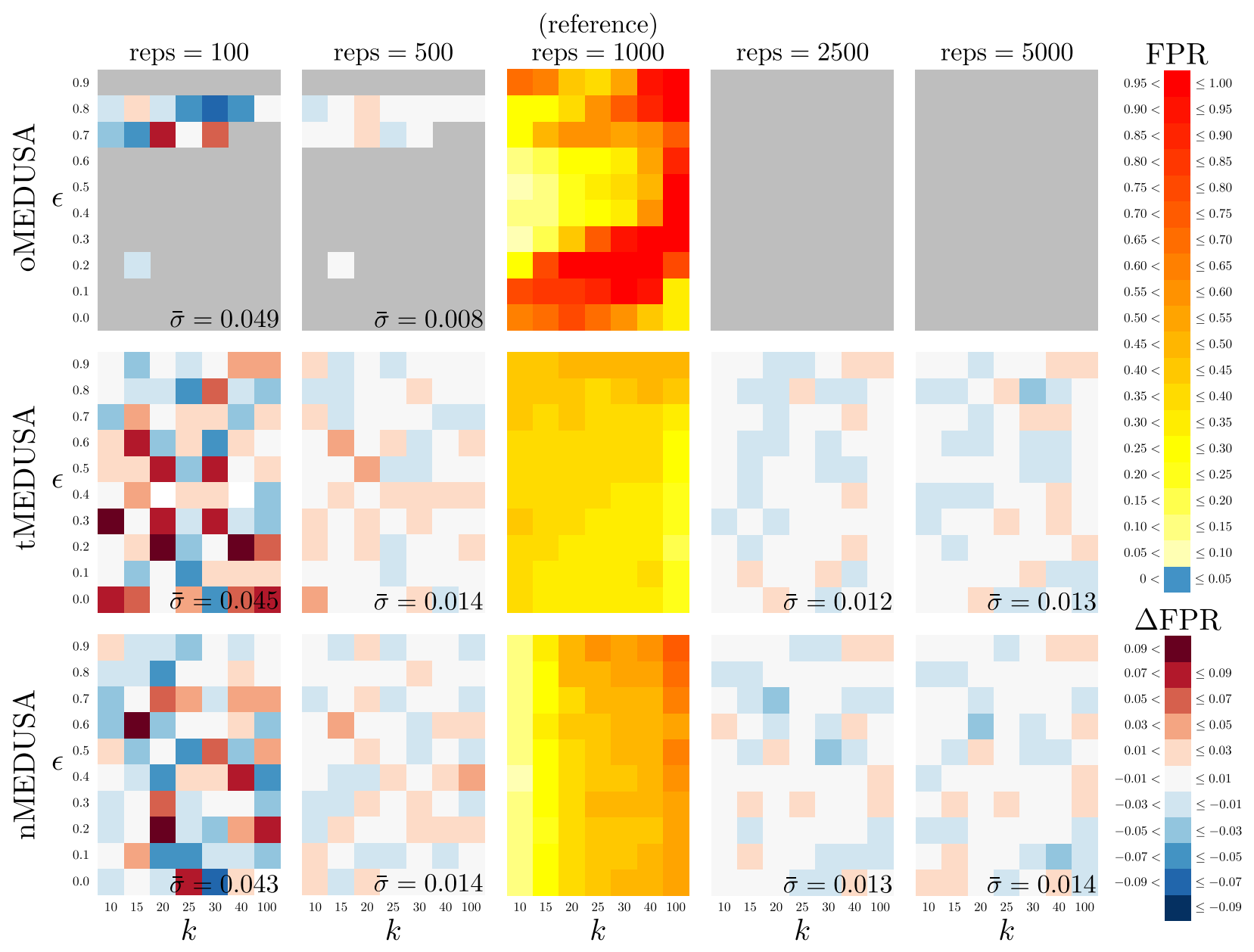

Figure S.4: Exploring the impact of Monte Carlo error on the complexity and intensity of estimated Type I error rates. The five panels in each row summarize results for one algorithm (oMEDUSA, tMEDUSA, and nMEDUSA, from top to bottom), the three panels in each column summarize results based on the number of replicates (with 100 to 5,000 simulated trees, from left to right). The center column - which is reproduced from Figure 2 (the right-most column, with $N=10,000$ species) - summarizes the Type I error rates based on 1,000 simulated trees, where the cells of each panel are colored to reflect the frequency with which spurious diversification-rate shifts were inferred (see FPR legend). These results serve as a reference to explore the impact of the number of simulated replicates on the inferred patterns and overall rates of Type I error. In the other columns, the cells within each panel are colored to reflect the difference in the estimated Type I error rate relative to that of its corresponding reference cell (see $\Delta$ FPR legend). The standard deviation, $\sigma$, of the Type I error rate is summarized in the lower right of each panel. These experiments suggest that $\sim 500$ replicates are sufficient for precise estimates of Type I error rates. For detailed results, see Table S.3. 
bioRxiv preprint doi: https://doi.org/10.1101/011452; this version posted November 15, 2014. The copyright holder for this preprint (which was not certified by peer review) is the author/funder, who has granted bioRxiv a license to display the preprint in perpetuity. It is made available under aCC-BY-ND 4.0 International license.

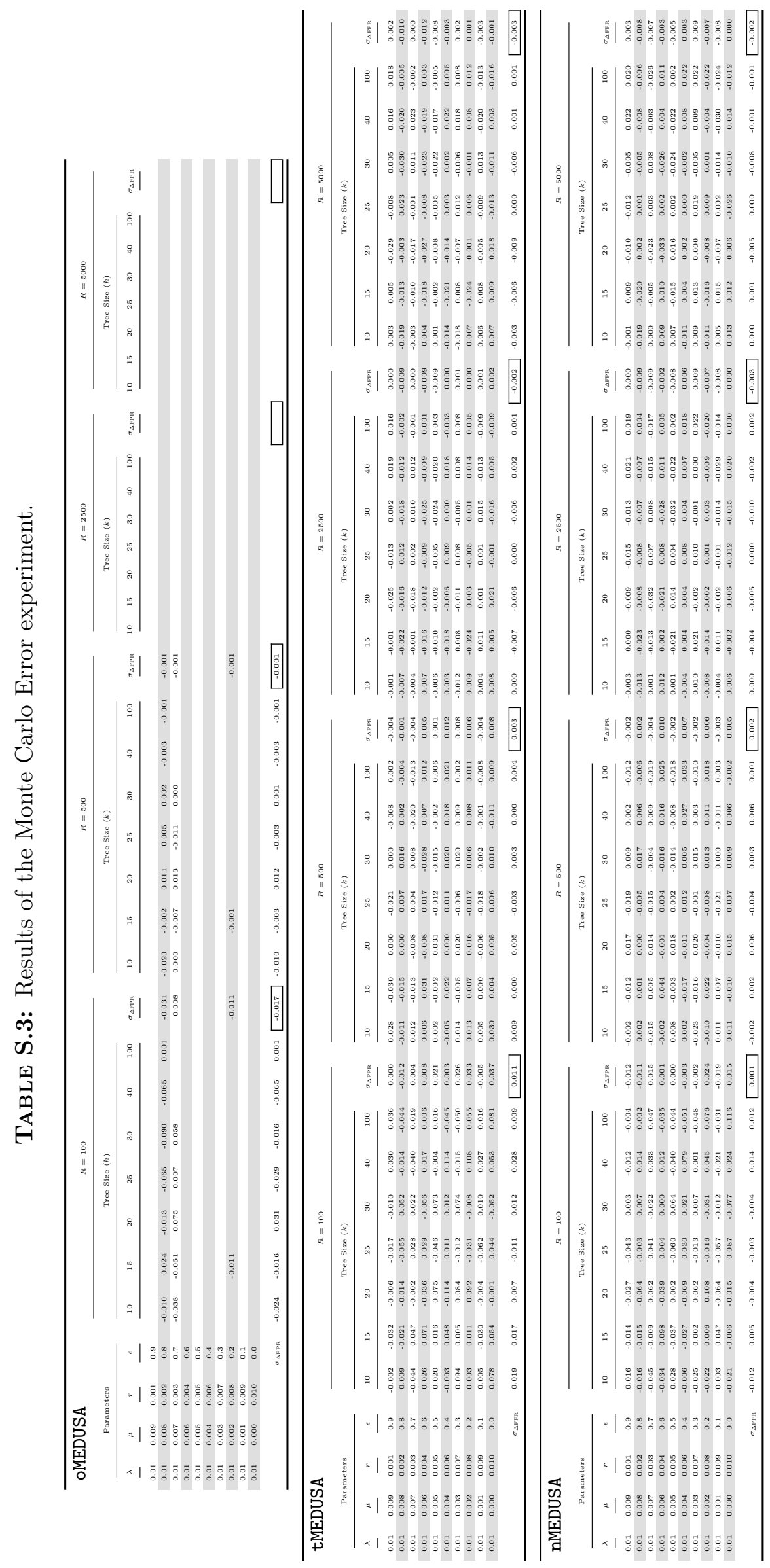

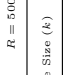




\section{S.5 The EFfeCt of ABsolute Diversification RATes on Type I ERror (STEM Shifts)}
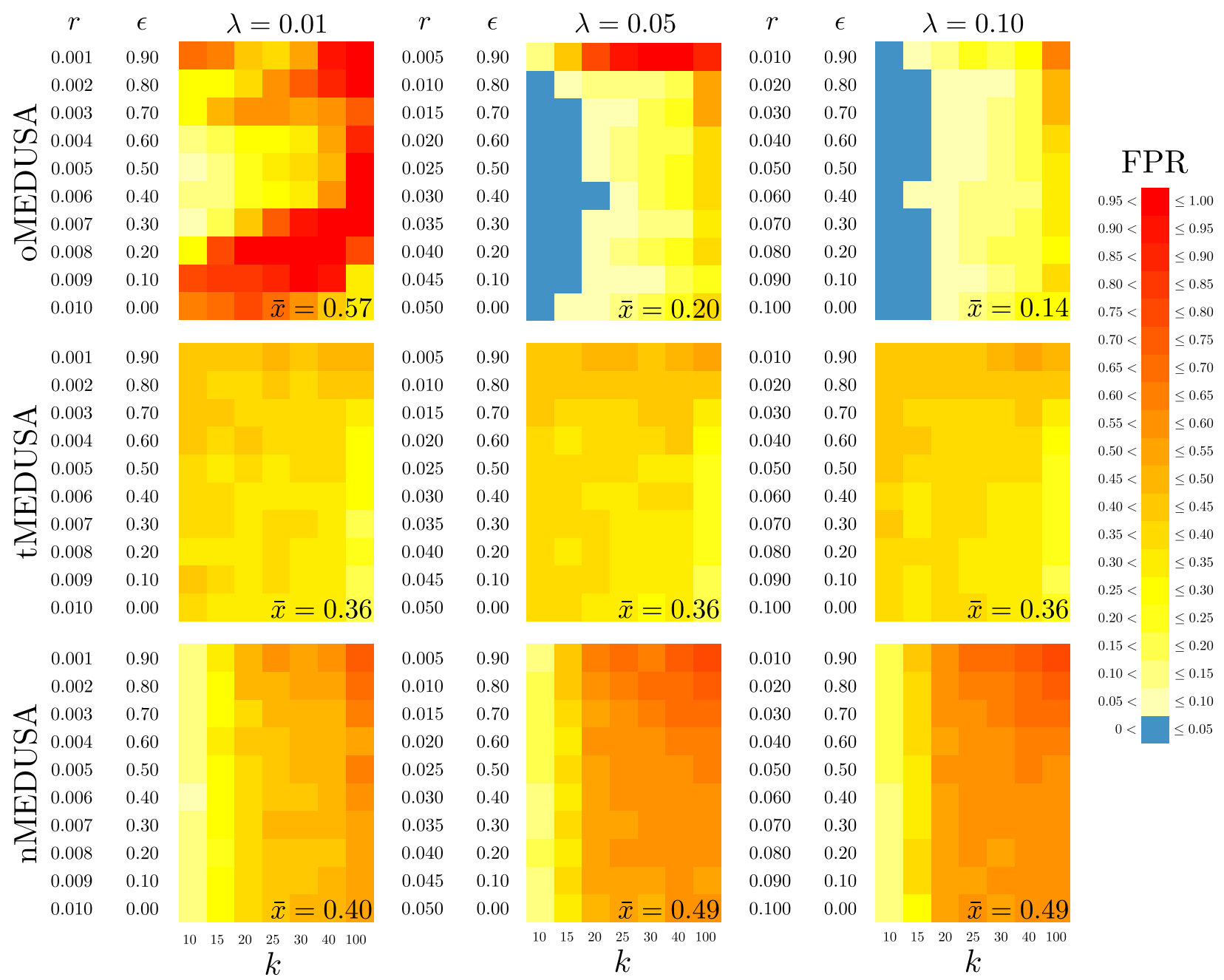

Figure S.5: The effect of increasing the absolute diversification rate on Type I error: maintaining consistent relative-extinction rates. The three panels in each row summarize results for one algorithm (oMEDUSA, tMEDUSA, and nMEDUSA from top to bottom), the panels in each column summarize results for one absolute speciation rate $(\lambda=0.01,0.05,0.10$, from left to right). Trees with $N=10,000$ species were simulated under a constant-rate birth-death process using the parameters described in Table S.4. For the absolute rates explored here, we specified a set of extinction rates, $\mu$, such that the set of relative-extinction rates, $\epsilon=\{0.00,0.01, \ldots, 0.90\}$ are consistent with those used in the initial simulation (Figure 2, reproduced here in the left column). Within each panel, we plot the number of terminal lineages, $k$, against the diversification rate, $r$. The cells within each panel are colored to reflect the frequency with which spurious diversificationrate shifts were inferred (see legend). The average Type I error rate is summarized in the lower right of each panel. 
bioRxiv preprint doi: https://doi.org/10.1101/011452; this version posted November 15,2014 . The copyright holder for this preprint (which was not certified by peer review) is the author/funder, who has granted bioRxiv a license to display the preprint in perpetuity. It is made available under aCC-BY-ND 4.0 International license.

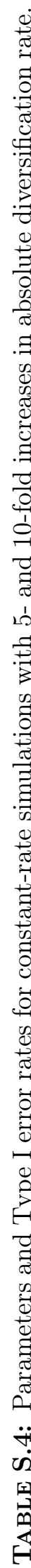

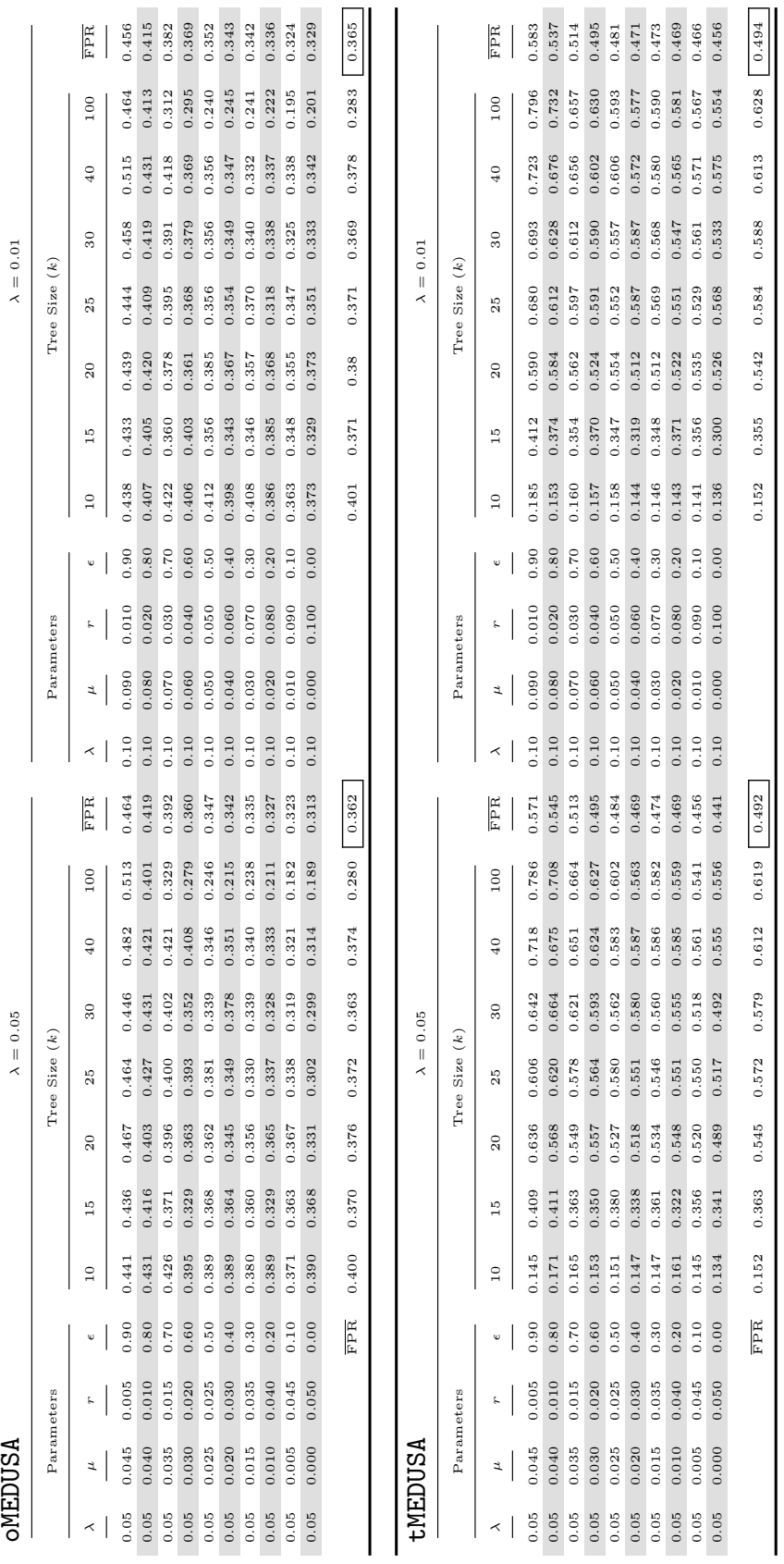



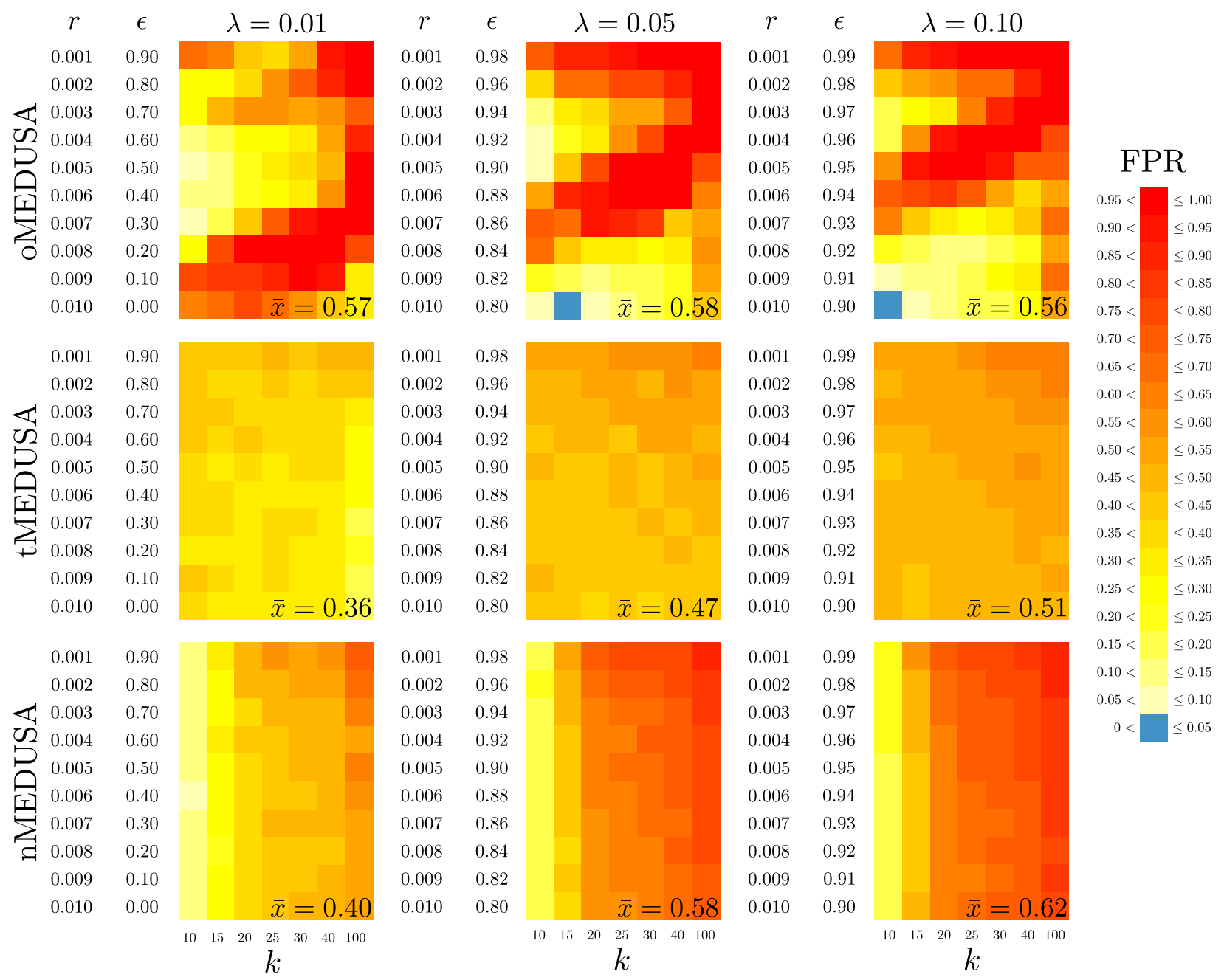

FIGURE S.6: The effect of increasing the absolute diversification rate on Type I error: maintaining consistent net-diversification rates. The three panels in each row summarize results for one algorithm (oMEDUSA, tMEDUSA, and nMEDUSA from top to bottom), the panels in each column summarize results for one absolute speciation rate $(\lambda=0.01,0.05,0.10$, from left to right). Trees with $N=10,000$ species were simulated under a constant-rate birth-death process using the parameters described in Table S.5. For the absolute rates explored here, we specified a set of extinction rates, $\mu$, such that the set of net-diversification rates, $r=\{0.001,0.002, \ldots, 0.010\}$ are consistent with those used in the initial simulation (Figure 2, reproduced here in the left column). Within each panel, we plot the number of terminal lineages, $k$, against the diversification rate, $r$. The cells within each panel are colored to reflect the frequency with which spurious diversificationrate shifts were inferred (see legend). The average Type I error rate is summarized in the lower right of each panel. 
bioRxiv preprint doi: https://doi.org/10.1101/011452; this version posted November 15,2014 . The copyright holder for this preprint (which was not certified by peer review) is the author/funder, who has granted bioRxiv a license to display the preprint in perpetuity. It is made available under aCC-BY-ND 4.0 International license.

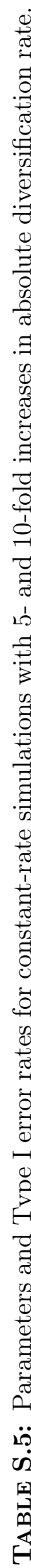

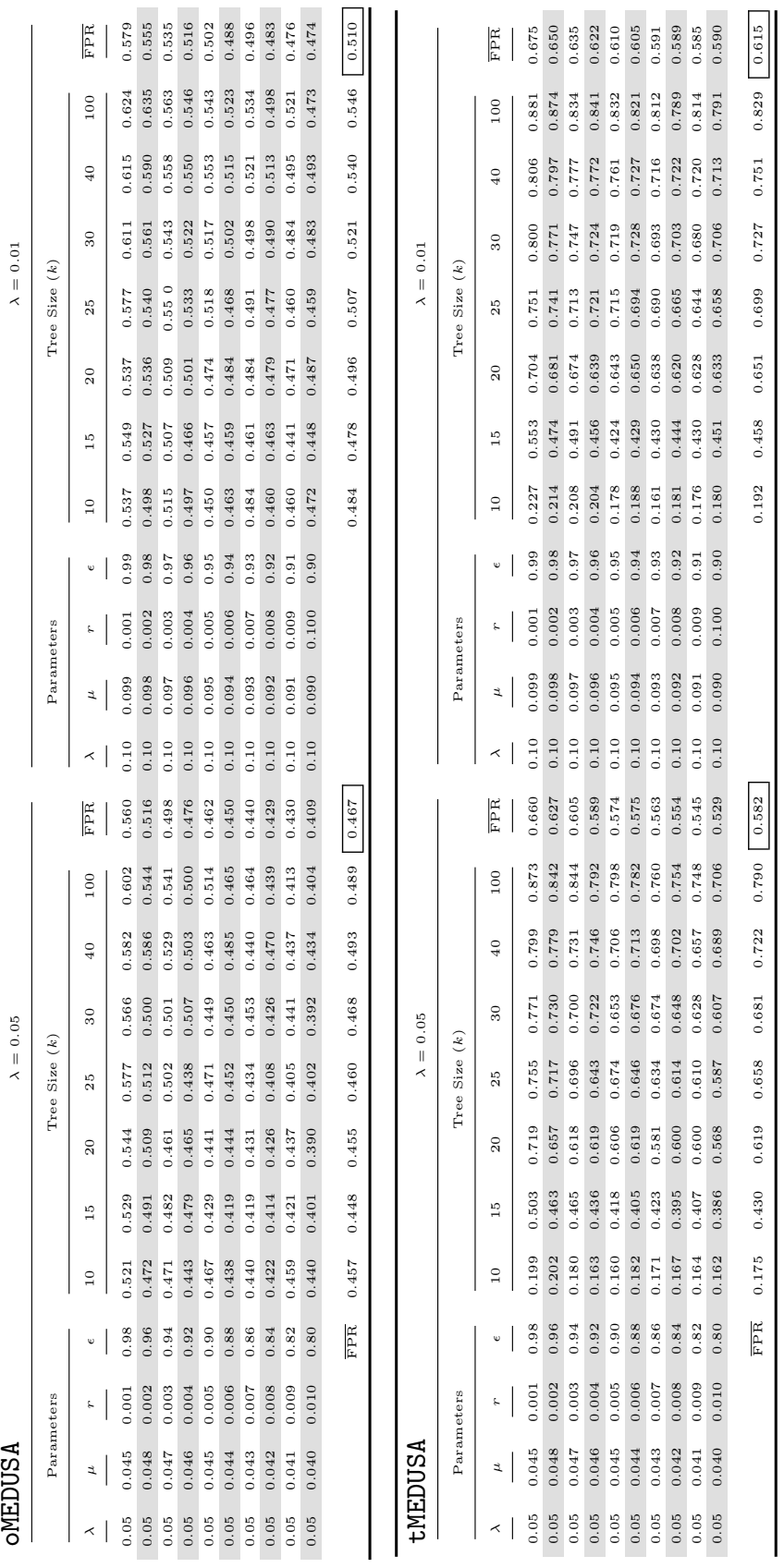


bioRxiv preprint doi: https://doi.org/10.1101/011452; this version posted November 15,2014 . The copyright holder for this preprint (which was not certified by peer review) is the author/funder, who has granted bioRxiv a license to display the preprint in perpetuity. It is made available under aCC-BY-ND 4.0 International license. 
bioRxiv preprint doi: https://doi.org/10.1101/011452; this version posted November 15, 2014. The copyright holder for this preprint (which was not certified by peer review) is the author/funder, who has granted bioRxiv a license to display the preprint in perpetuity. It is made available under aCC-BY-ND 4.0 International license.

S.6 Exploring THRESHOLD EFFECTS on TyPe I ERror RATES (STEM SHifts)

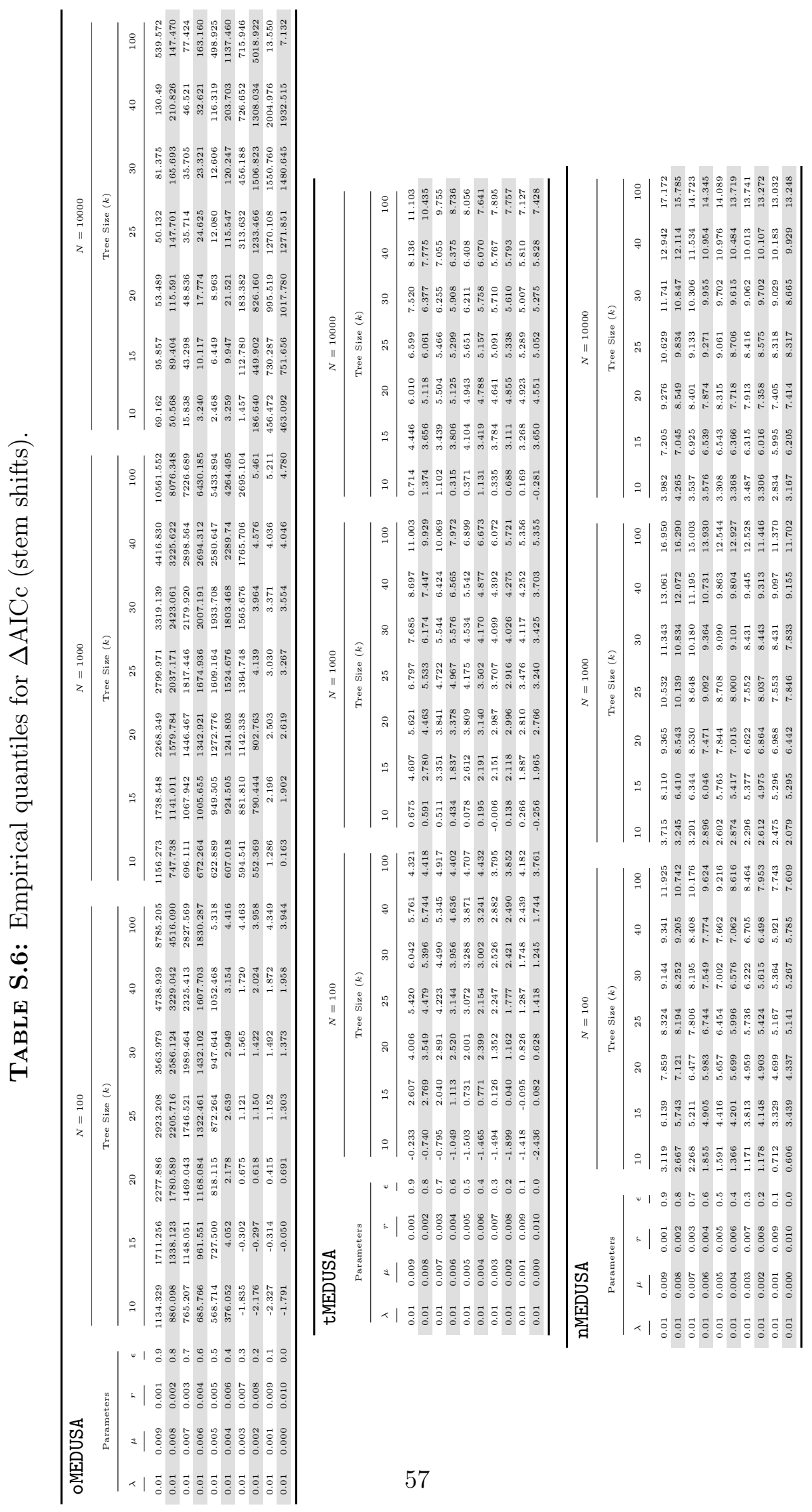


bioRxiv preprint doi: https://doi.org/10.1101/011452; this version posted November 15,2014 . The copyright holder for this preprint (which was not certified by peer review) is the author/funder, who has granted bioRxiv a license to display the preprint in perpetuity. It is made available under aCC-BY-ND 4.0 International license. 
bioRxiv preprint doi: https://doi.org/10.1101/011452; this version posted November 15, 2014. The copyright holder for this preprint (which was not certified by peer review) is the author/funder, who has granted bioRxiv a license to display the preprint in perpetuity. It is made available under aCC-BY-ND 4.0 International license.

S.7 Exploring Type I ERror RATES FOR COMPlETE SPECIES TREes (STEM Shifts)

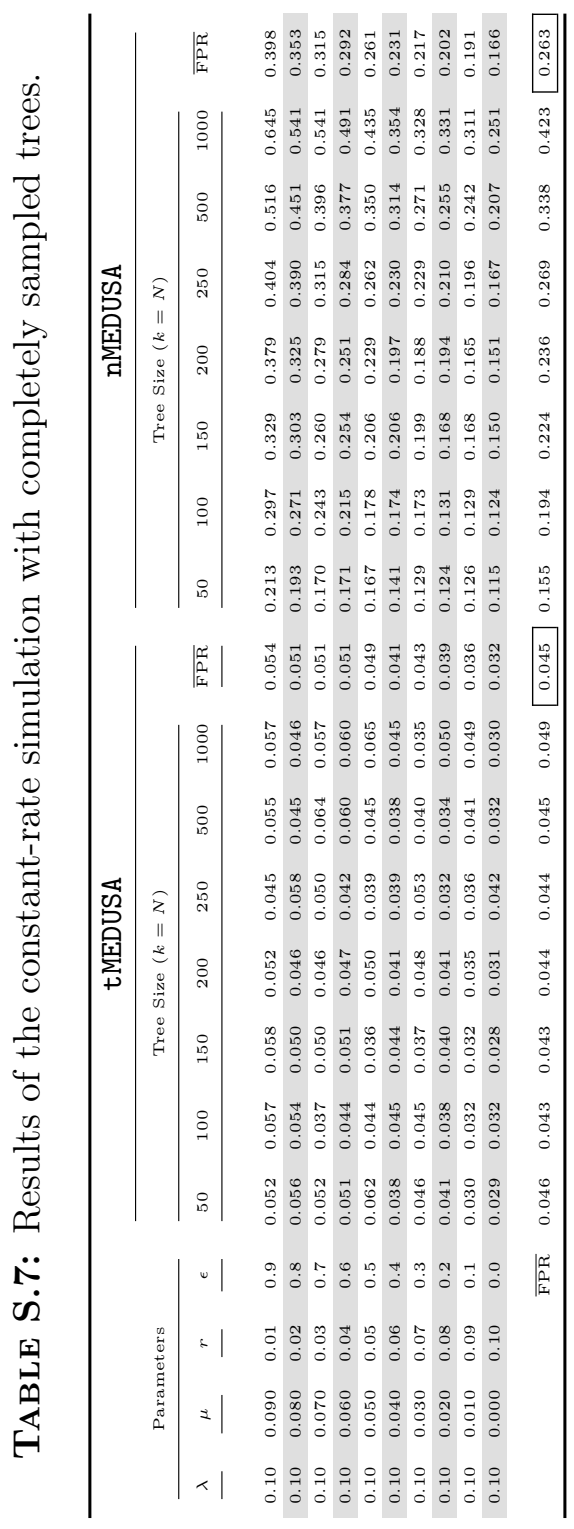




\section{S.8 Assessing the impaCt of terminal UnResolved Clades (Stem Shifts)}

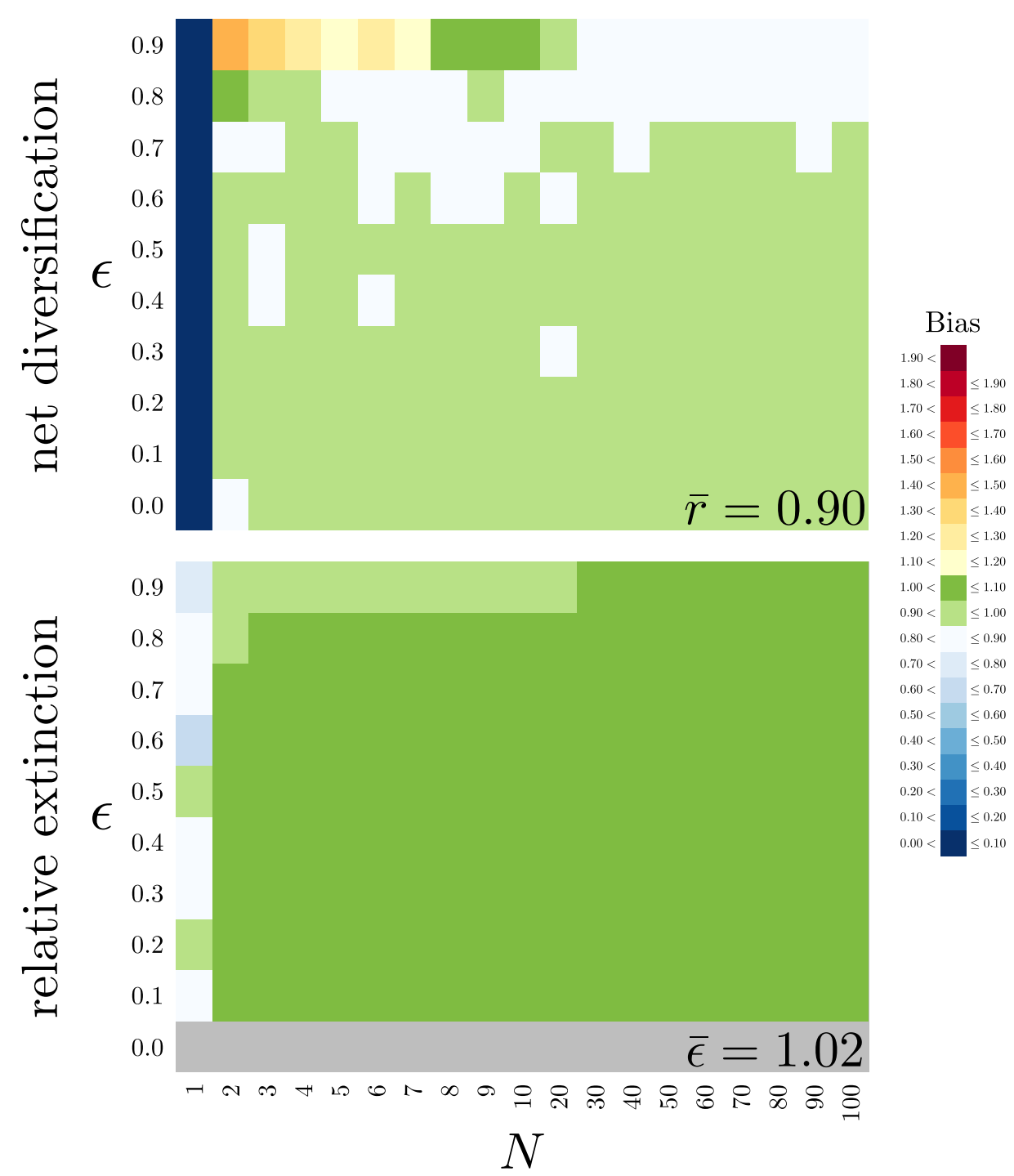

Figure S.7: The size of terminal unresolved lineages effects bias in parameter estimates. Trees of size $N$ ranging from 1 to 1000 were simulated under a constant-rate birth-death process using parameters summarized in Tables S.8 and S.9. For each simulated tree, we estimated the net-diversification rate (upper panel) and relative-extinction rate (lower panel) using equation 2. Each panel plots lineage size, $N$, against the relative-extinction rate, $\epsilon$, used in the simulation. The cells within each panel are colored as a heat map reflecting the difference between the estimated and true rates (see Bias legend). The average bias in the parameter estimates is summarized in the lower right of each panel. 
bioRxiv preprint doi: https://doi.org/10.1101/011452; this version posted November 15, 2014. The copyright holder for this preprint (which was not certified by peer review) is the author/funder, who has granted bioRxiv a license to display the preprint in perpetuity. It is made available under aCC-BY-ND 4.0 International license.

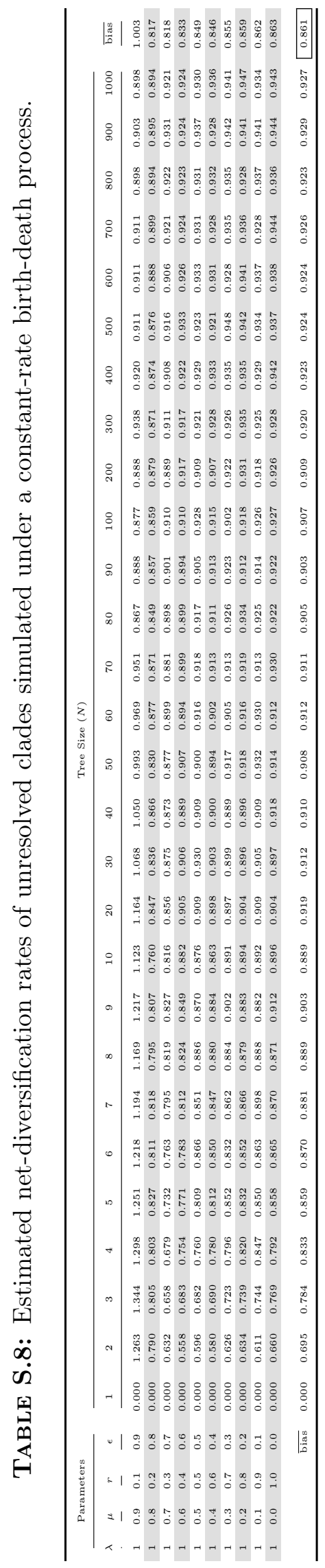


bioRxiv preprint doi: https://doi.org/10.1101/011452; this version posted November 15, 2014. The copyright holder for this preprint (which was not certified by peer review) is the author/funder, who has granted bioRxiv a license to display the preprint in perpetuity. It is made available under aCC-BY-ND 4.0 International license.

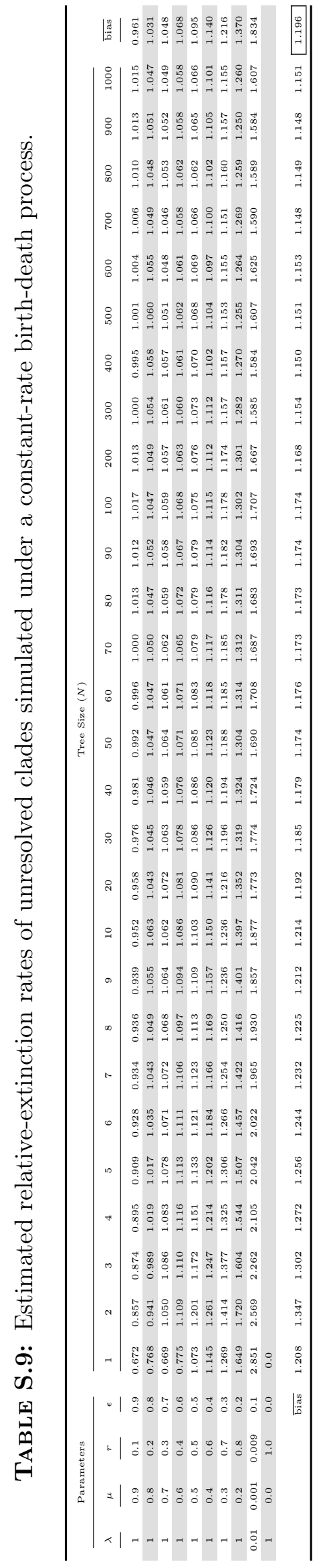




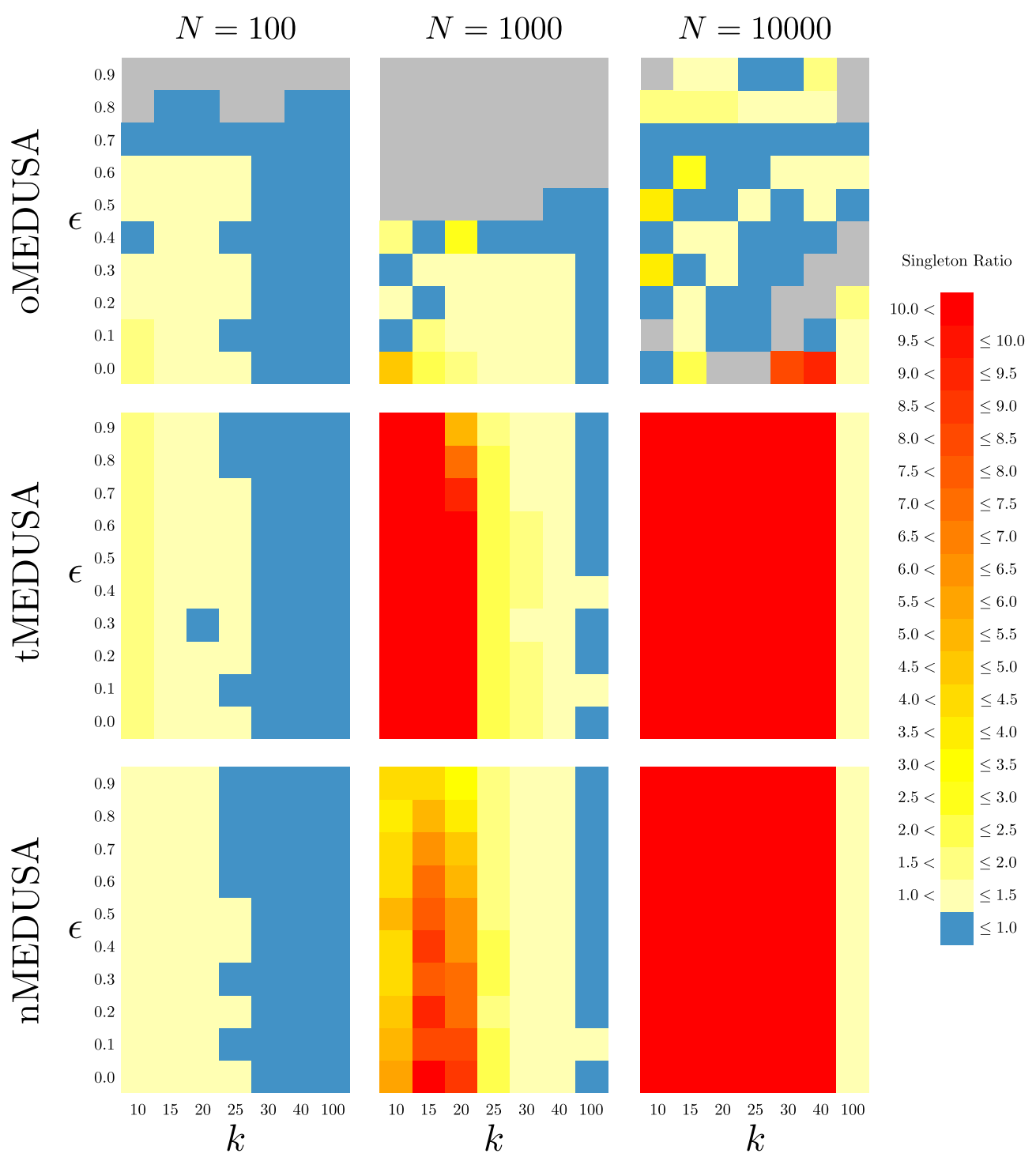

FiguRE S.8: Relative frequency of singleton terminal linaeages in trees with spurious rate shifts versus trees without rate shifts. The three panels in each row summarize results for one algorithm (oMEDUSA, tMEDUSA, and nMEDUSA, from top to bottom), the three panels in each column summarize results for one tree size (with $N=100,1,000,10,000$ species, from left to right). Within each panel, we plot the number of terminal lineages, $k$, against the relative-extinction rate used in the simulation. Trees were simulated under a constant-rate birth-death process using the parameters summarized in Table S.2. The cells within each panel are colored as a heat map reflecting the ratio of the frequency of 'singletons' (terminal lineages with a single species) in trees where MEDUSA incorrectly identified a rate shift versus the singleton frequency in trees where it correctly identified no rate shift (see Singleton Ratio legend). 
bioRxiv preprint doi: https://doi.org/10.1101/011452; this version posted November 15, 2014. The copyright holder for this preprint (which was not certified by peer review) is the author/funder, who has granted bioRxiv a license to display the preprint in perpetuity. It is made available under aCC-BY-ND 4.0 International license.

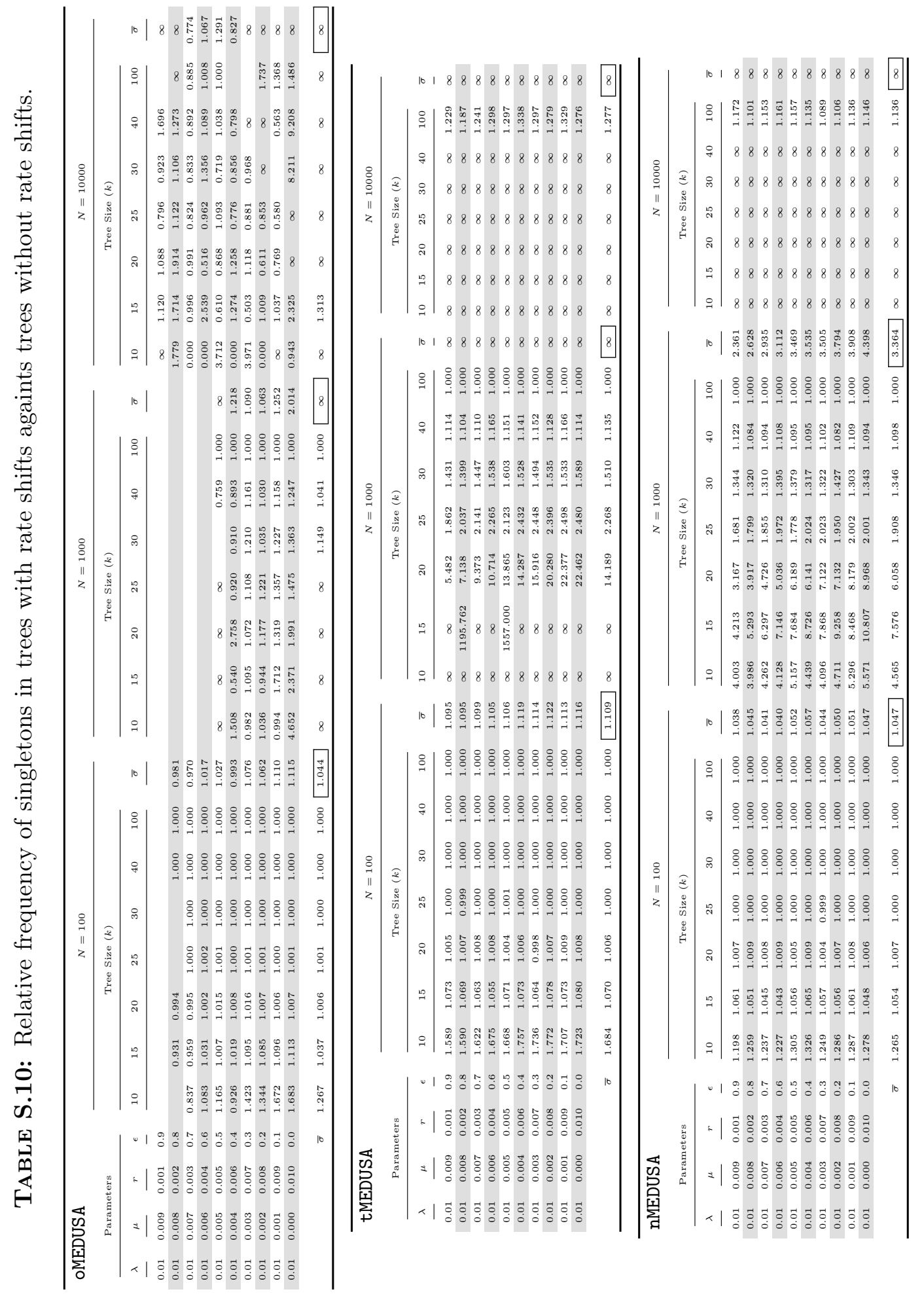


bioRxiv preprint doi: https://doi.org/10.1101/011452; this version posted November 15,2014 . The copyright holder for this preprint (which was not certified by peer review) is the author/funder, who has granted bioRxiv a license to display the preprint in perpetuity. It is made available under aCC-BY-ND 4.0 International license. 
bioRxiv preprint doi: https://doi.org/10.1101/011452; this version posted November 15,2014 . The copyright holder for this preprint (which was not certified by peer review) is the author/funder, who has granted bioRxiv a license to display the preprint in perpetuity. It is made available under aCC-BY-ND 4.0 International license.

S.9 Parameter estimation (STEM Shifts)
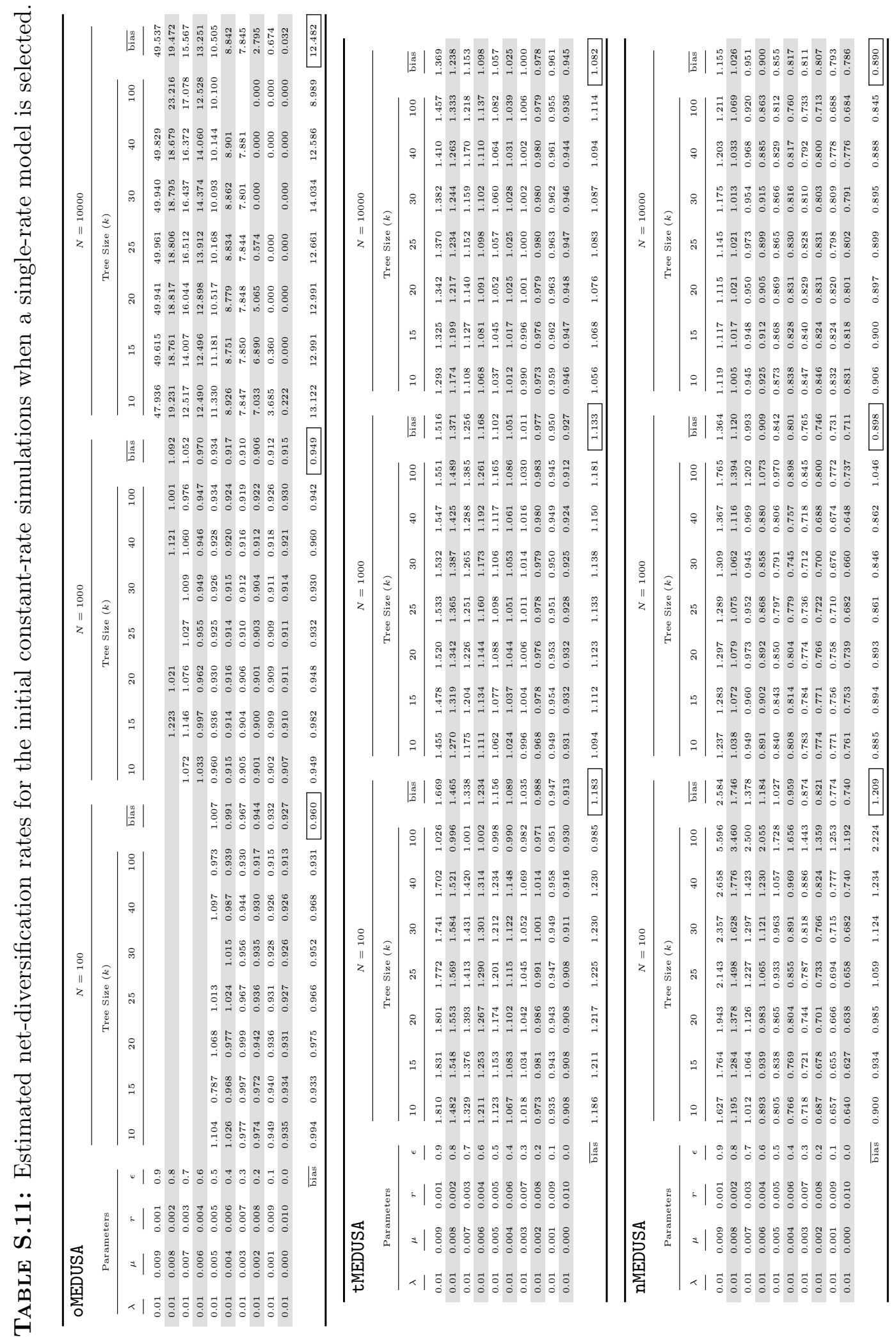

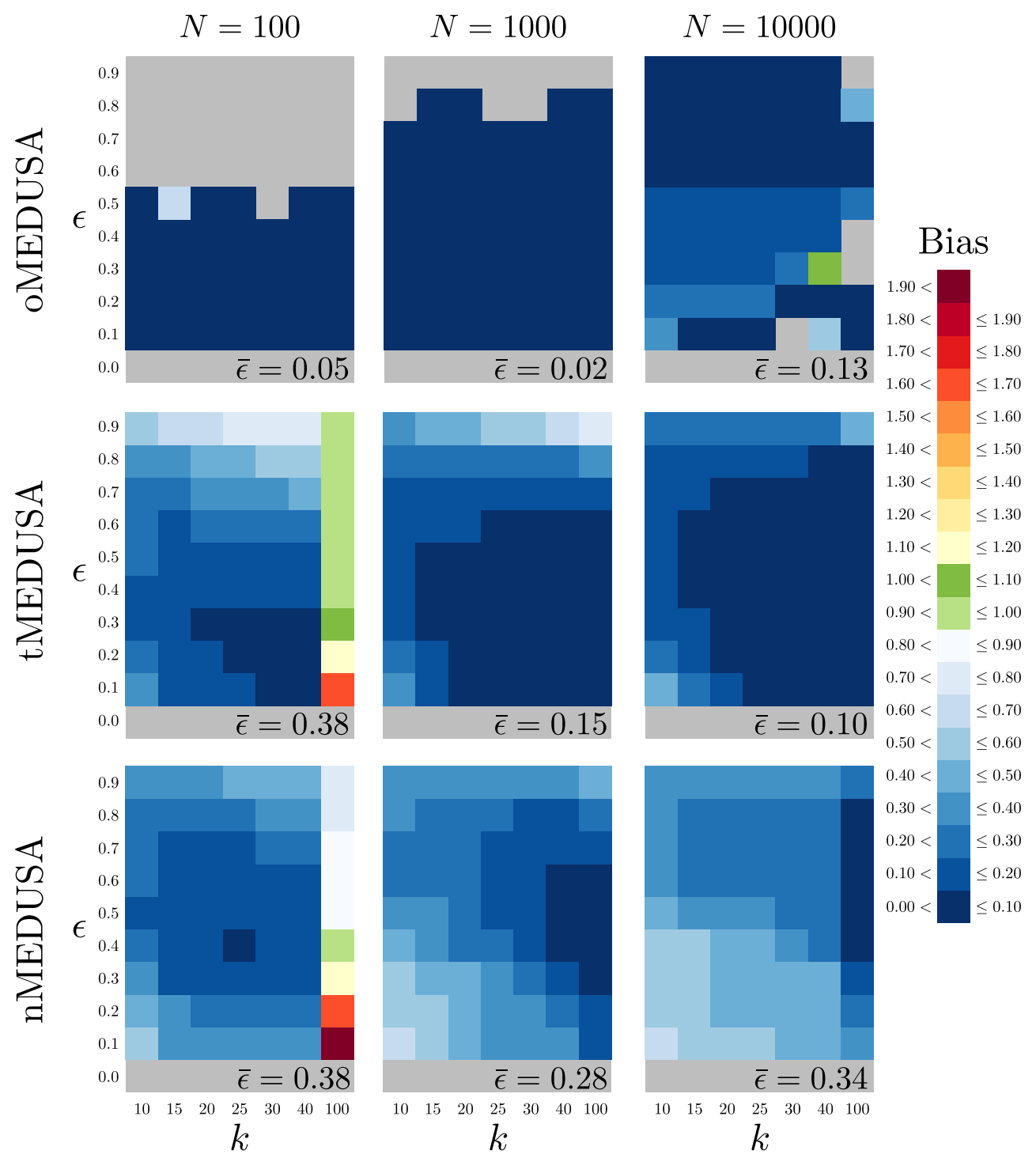

Figure S.9: Bias in estimated relative-extinction rate when a single-rate model is selected. The three panels in each row summarize results for one algorithm (oMEDUSA, tMEDUSA, and nMEDUSA, from top to bottom), the three panels in each column summarize results for one tree size (with $N=100,1,000,10,000$ species, from left to right). Within each panel, we plot the number of terminal lineages, $k$, against the relative-extinction rate used in the simulation. Trees were simulated under a constant-rate birth-death process using the parameters summarized in Table S.12. The cells within each panel are colored to reflect the average difference between the estimated and true relative-extinction rates when a one-rate model is correctly identified (see Bias legend). The average bias in estimates of relative extinction-rate is summarized in the lower right of each panel. 
bioRxiv preprint doi: https://doi.org/10.1101/011452; this version posted November 15, 2014. The copyright holder for this preprint (which was not certified by peer review) is the author/funder, who has granted bioRxiv a license to display the preprint in perpetuity. It is made available under aCC-BY-ND 4.0 International license.

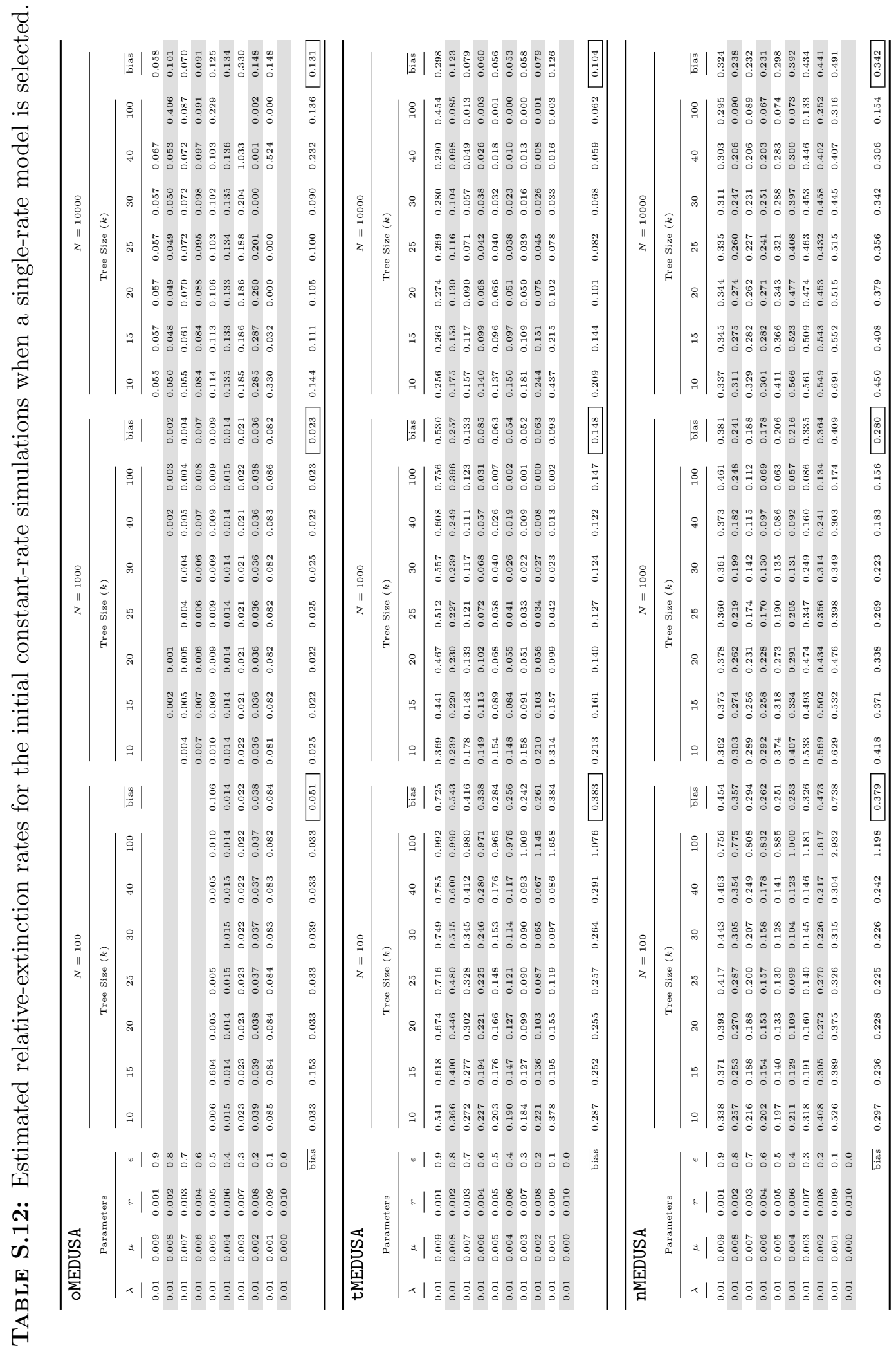




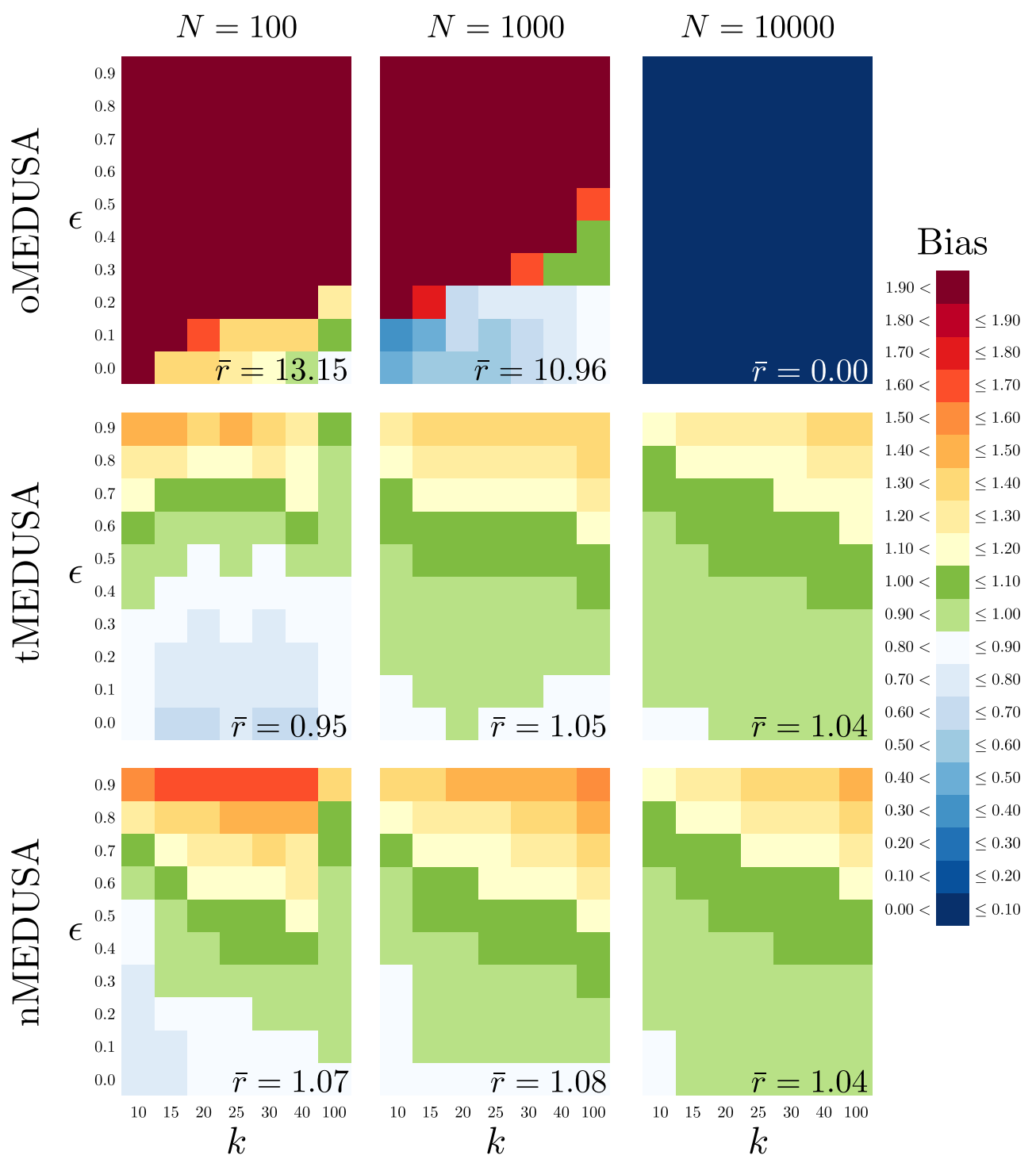

FiguRE S.10: Bias in estimated background net-diversification rates when a two-rate model is selected. The three panels in each row summarize results for one algorithm (oMEDUSA, tMEDUSA, and nMEDUSA, from top to bottom), the three panels in each column summarize results for one tree size (with $N=100,1,000,10,000$ species, from left to right). Within each panel, we plot the number of terminal lineages, $k$, against the relative-extinction rate used in the simulation. Trees were simulated under a constant-rate birth-death process using the parameters summarized in Table S.13. The cells within each panel are colored to reflect the average ratio of the estimated vs. true background net-diversification rate when a two-rate model is incorrectly identified (see Bias legend). The average bias in the inferred background net-diversification rate is summarized in the lower right of each panel. 
bioRxiv preprint doi: https://doi.org/10.1101/011452; this version posted November 15,2014 . The copyright holder for this preprint (which was not certified by peer review) is the author/funder, who has granted bioRxiv a license to display the preprint in perpetuity. It is made available under aCC-BY-ND 4.0 International license.
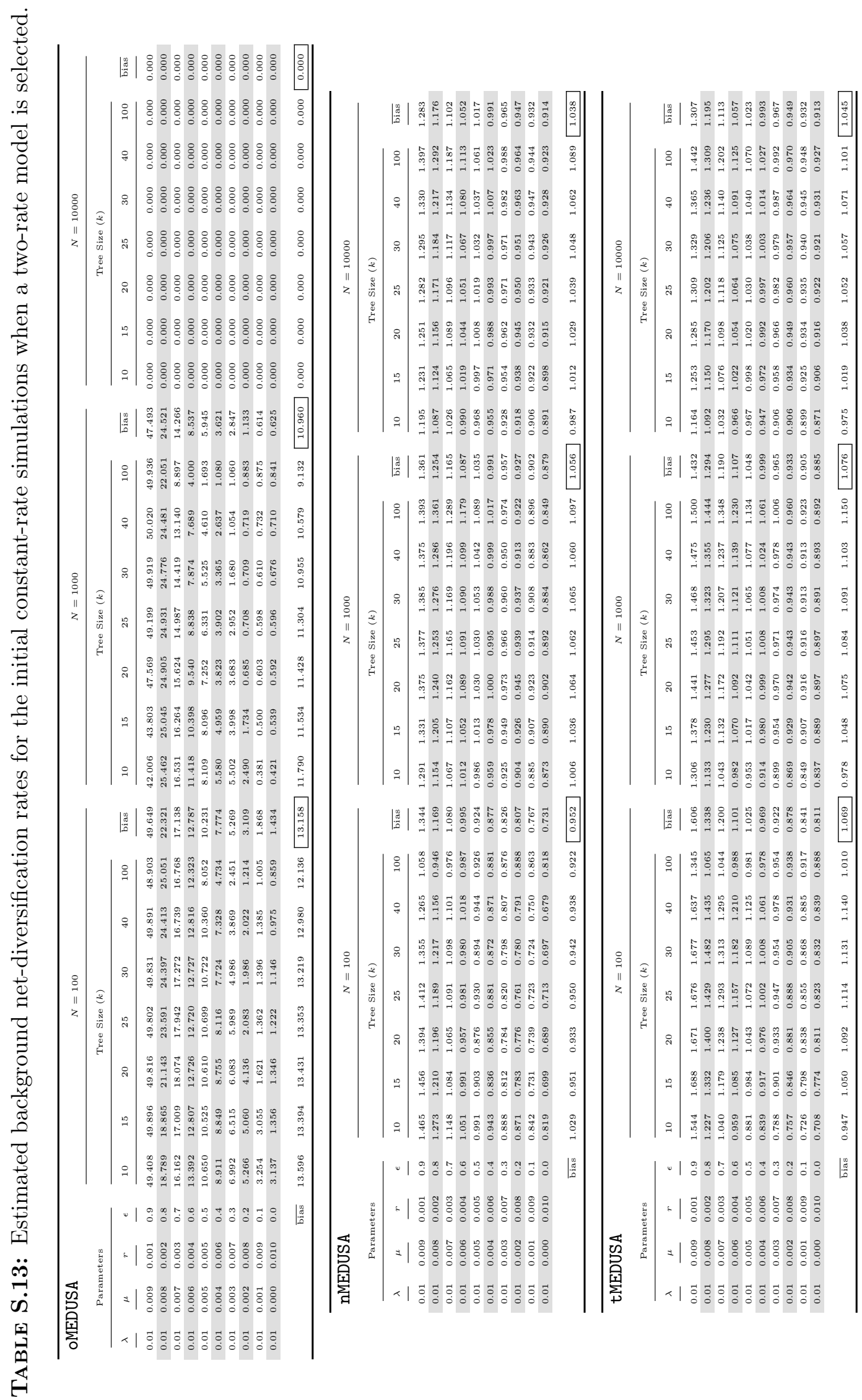

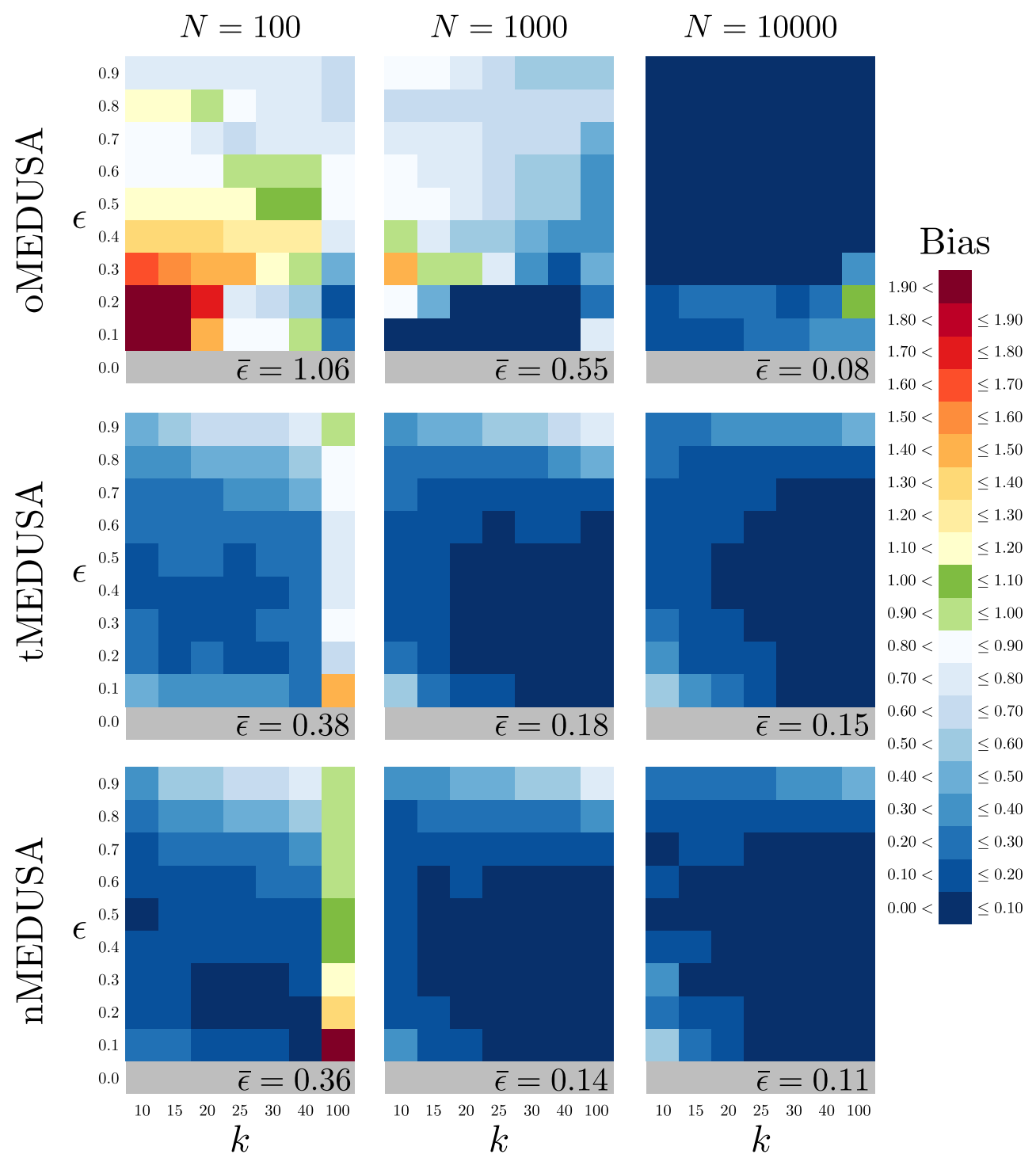

FIgURE S.11: Bias in estimated background relative-extinction rates when a two-rate model is selected. The three panels in each row summarize results for one algorithm (oMEDUSA, tMEDUSA, and nMEDUSA, from top to bottom), the three panels in each column summarize results for one tree size (with $N=100,1,000,10,000$ species, from left to right). Within each panel, we plot the number of terminal lineages, $k$, against the relative-extinction rate used in the simulation. Trees were simulated under a constant-rate birth-death process using the parameters summarized in Table S.14. The cells within each panel are colored to reflect the average ratio of the estimated vs. true background relative-extinction rate when a two-rate model is incorrectly identified (see Bias legend). The average bias in the inferred background relative-extinction rate is summarized in the lower right of each panel. 
bioRxiv preprint doi: https://doi.org/10.1101/011452; this version posted November 15,2014 . The copyright holder for this preprint (which was not certified by peer review) is the author/funder, who has granted bioRxiv a license to display the preprint in perpetuity. It is made available under aCC-BY-ND 4.0 International license.

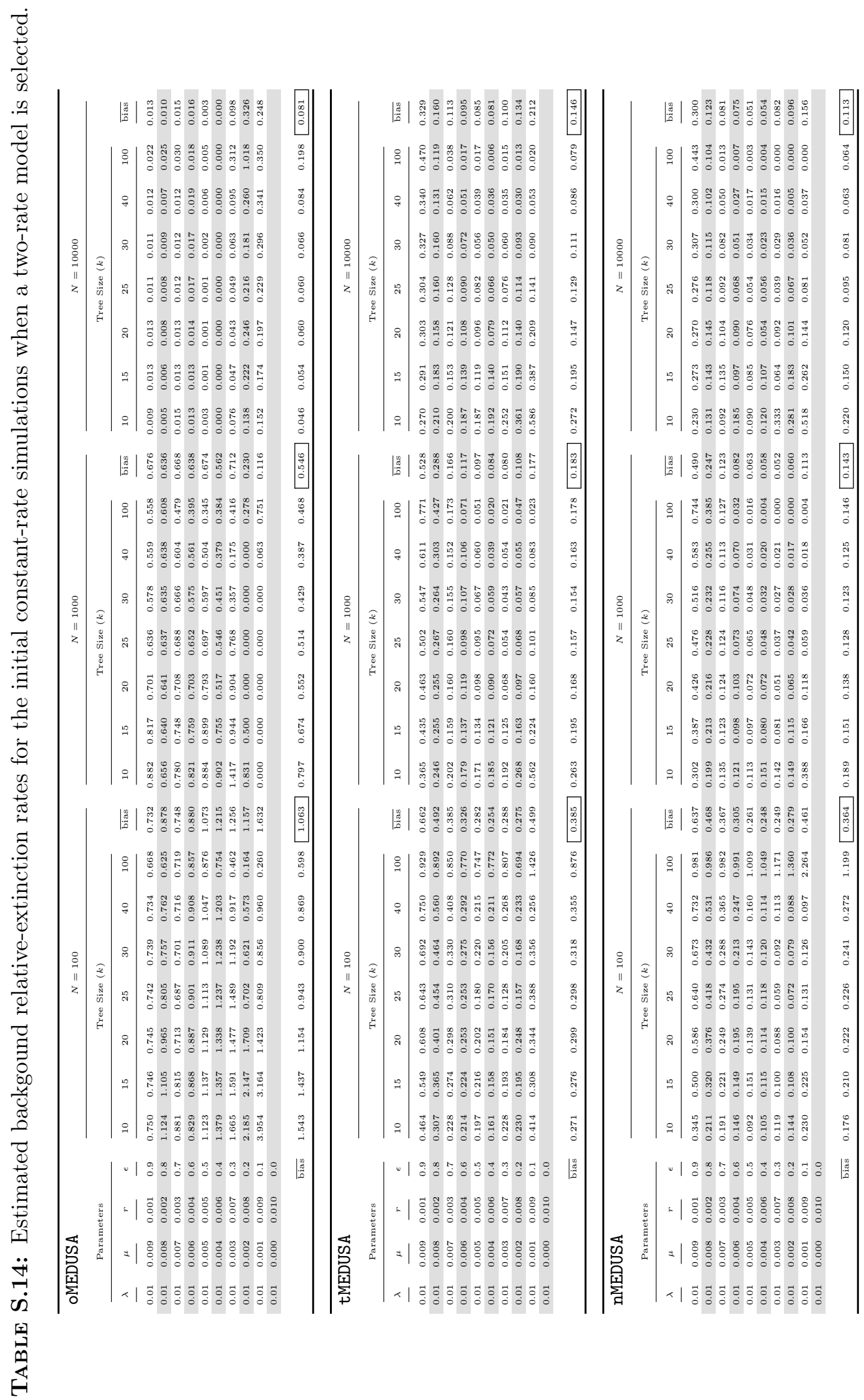


bioRxiv preprint doi: https://doi.org/10.1101/011452; this version posted November 15,2014 . The copyright holder for this preprint (which was not certified by peer review) is the author/funder, who has granted bioRxiv a license to display the preprint in perpetuity. It is made available under aCC-BY-ND 4.0 International license.

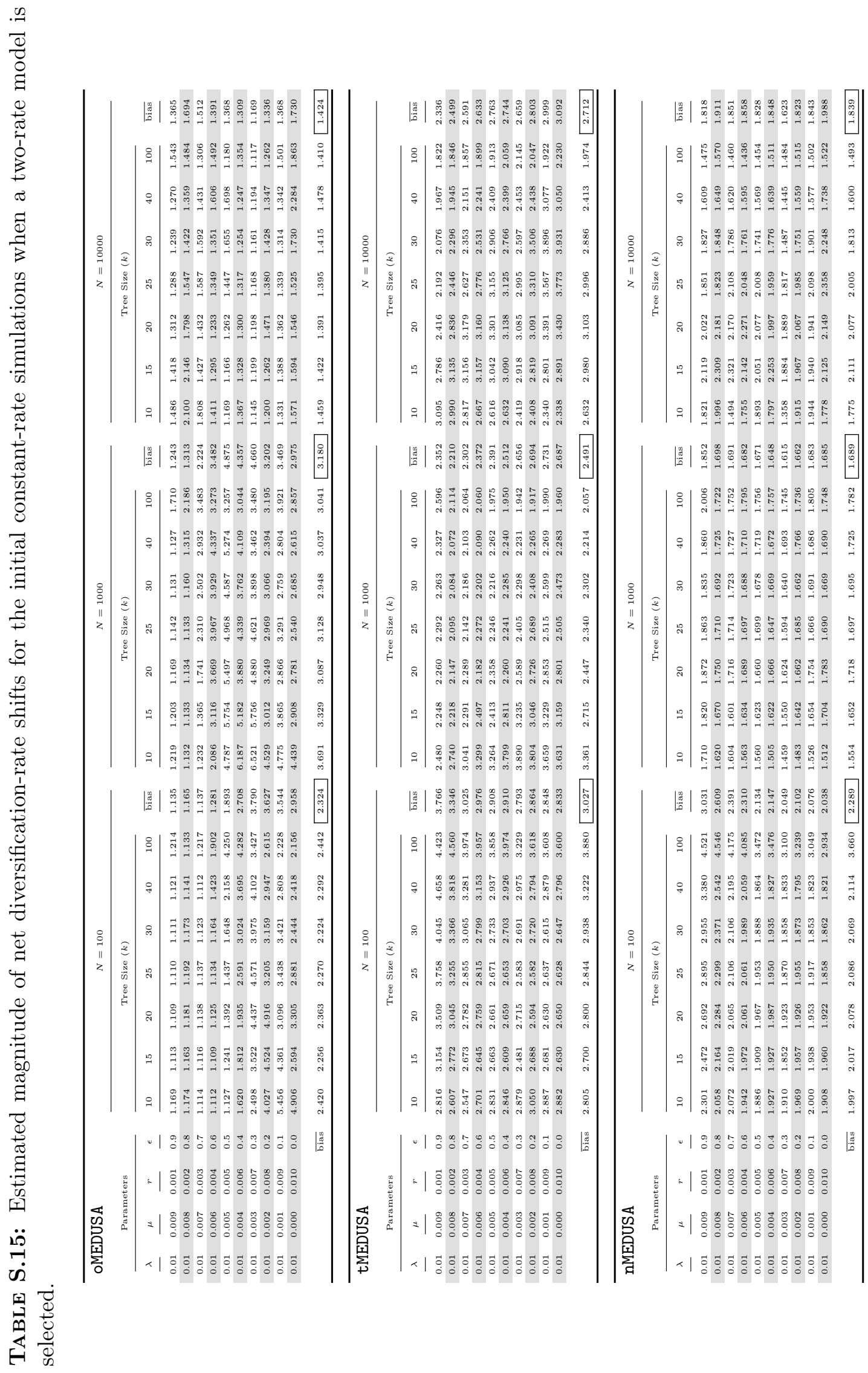



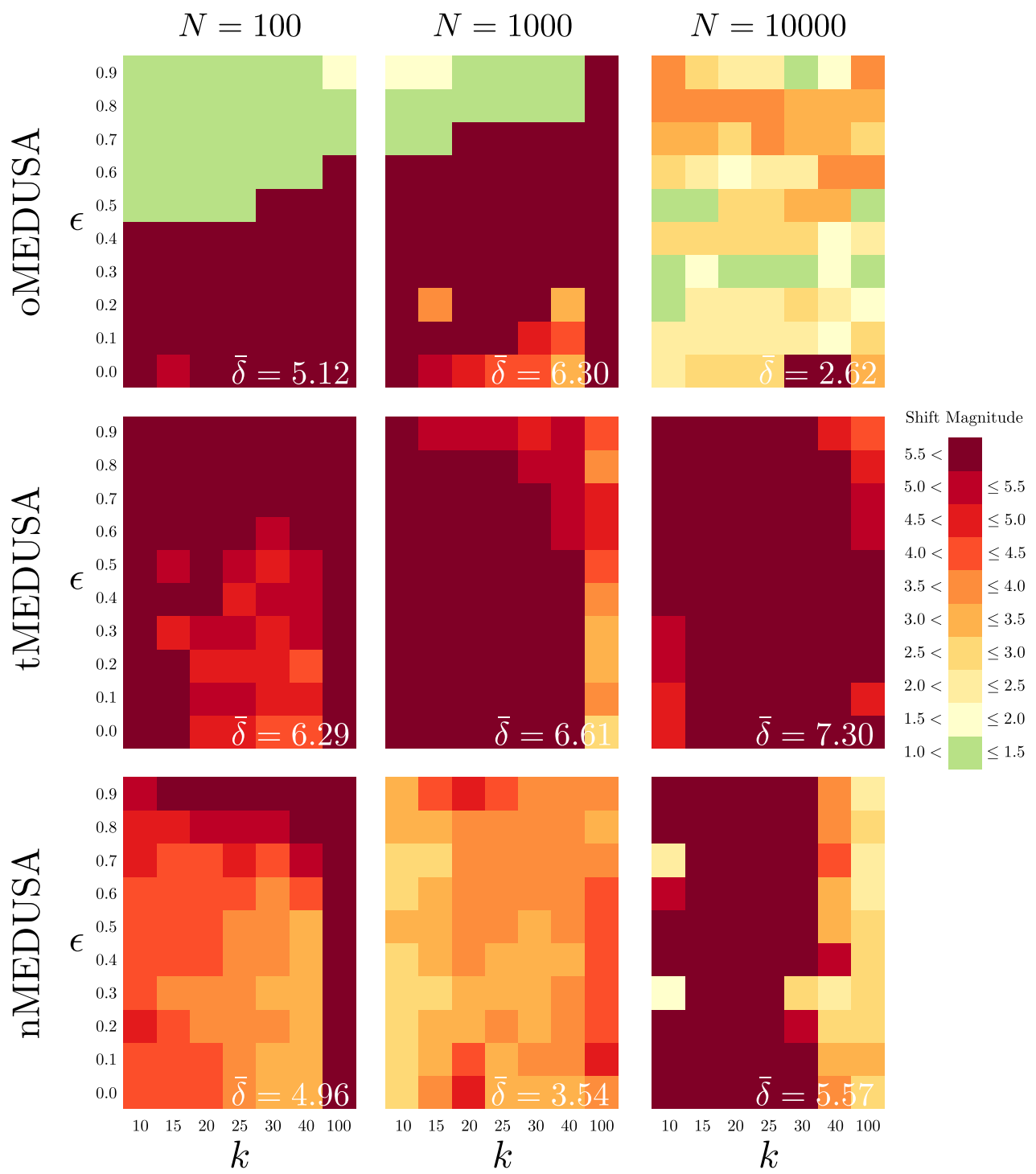

Figure S.12: 95\% quantile of shift magnitude in net-diversification rate when a tworate model is selected. The three panels in each row summarize results for one algorithm (oMEDUSA, tMEDUSA, and nMEDUSA, from top to bottom), the three panels in each column summarize results for one tree size (with $N=100,1,000,10,000$ species, from left to right). Within each panel, we plot the number of terminal lineages, $k$, against the relative-extinction rate used in the simulation. Trees were simulated under a constant-rate birth-death process using the parameters summarized in Table S.16. The cells within each panel are colored to reflect the $95 \%$ quantile of the magnitude of the inferred shift in net-diversification rate when a two-rate model is incorrectly identified (see Shift Magnitude legend). In order to accommodate the bimodal distribution of rate shifts, the magnitude of each net-diversification-rate shift was computed as the ratio of the higher rate to the lower rate, regardless of which rate corresponded to the background-rate category. The average $95 \%$ quantile of the magnitude of shifts in net-diversification rate shift is summarized in the lower right of each panel. 
bioRxiv preprint doi: https://doi.org/10.1101/011452; this version posted November 15,2014 . The copyright holder for this preprint (which was not certified by peer review) is the author/funder, who has granted bioRxiv a license to display the preprint in perpetuity. It is made available under aCC-BY-ND 4.0 International license.

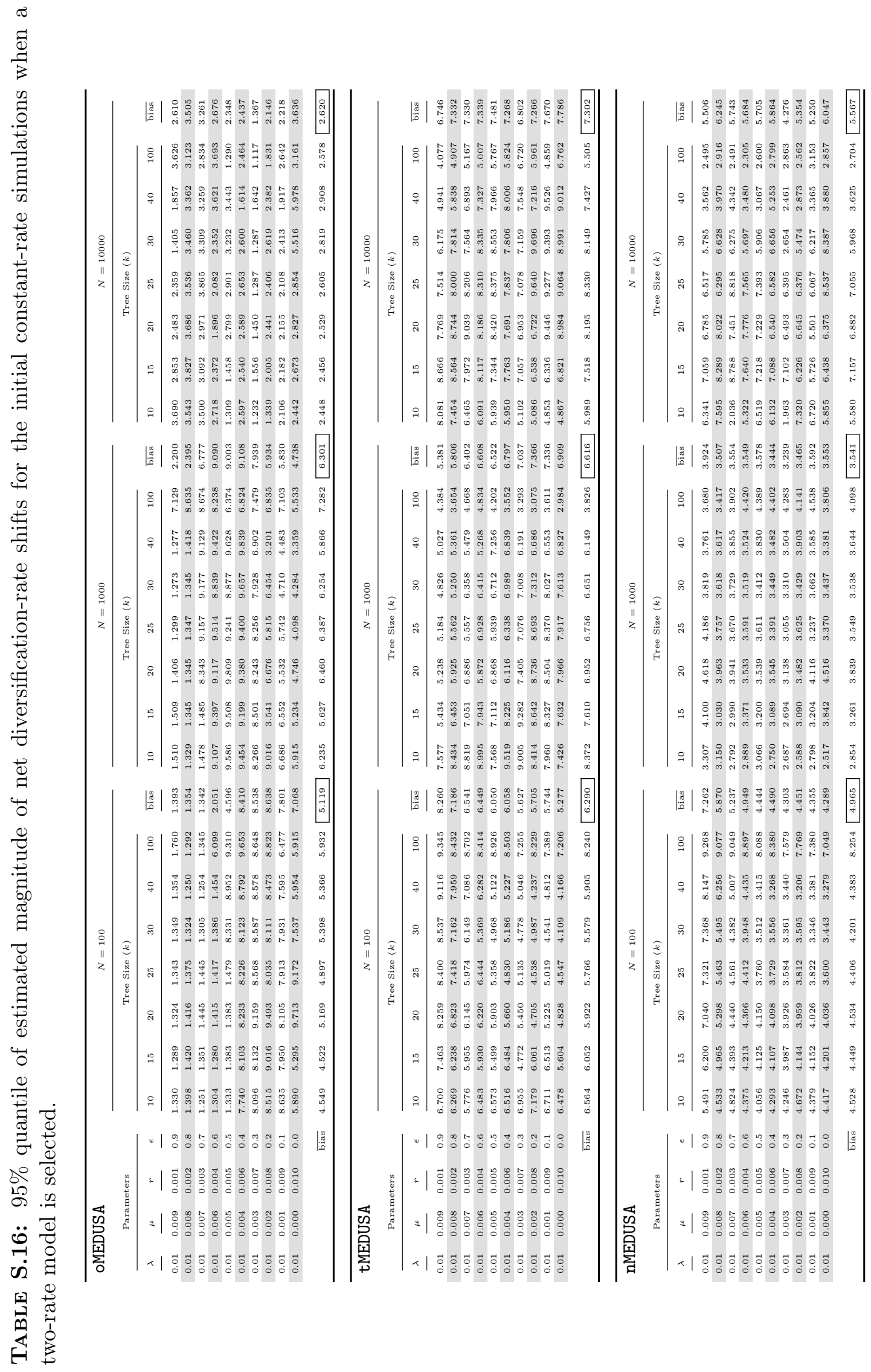



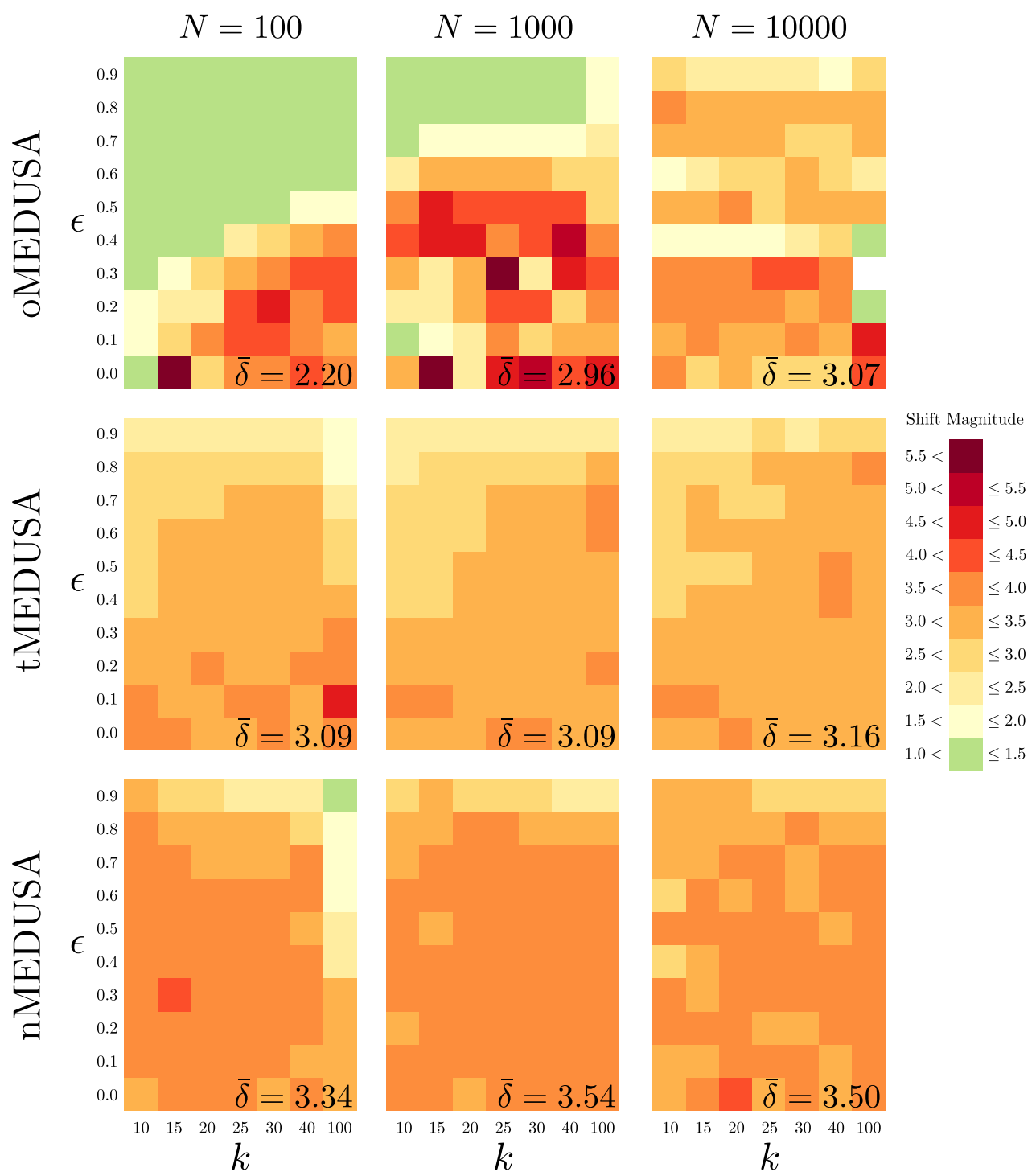

Figure S.13: Magnitude of shift in relative-extinction rate when a two-rate model is selected. The three panels in each row summarize results for one algorithm (oMEDUSA, tMEDUSA, and nMEDUSA, from top to bottom), the three panels in each column summarize results for one tree size (with $N=100,1,000,10,000$ species, from left to right). Within each panel, we plot the number of terminal lineages, $k$, against the relative-extinction rate used in the simulation. Trees were simulated under a constant-rate birth-death process using the parameters summarized in Table S.17. The cells within each panel are colored to reflect the average magnitude of the inferred shift in relative-extinction rate when a two-rate model is incorrectly identified (see Shift Magnitude legend). In order to accommodate the bimodal distribution of rate shifts, the magnitude of each relativeextinction-rate shift was computed as the ratio of the higher rate to the lower rate, regardless of which rate corresponded to the background-rate category. The average magnitude of shifts in relative-extinction rate shift is summarized in the lower right of each panel. 
bioRxiv preprint doi: https://doi.org/10.1101/011452; this version posted November 15, 2014. The copyright holder for this preprint (which was not certified by peer review) is the author/funder, who has granted bioRxiv a license to display the preprint in perpetuity. It is made available under aCC-BY-ND 4.0 International license. 

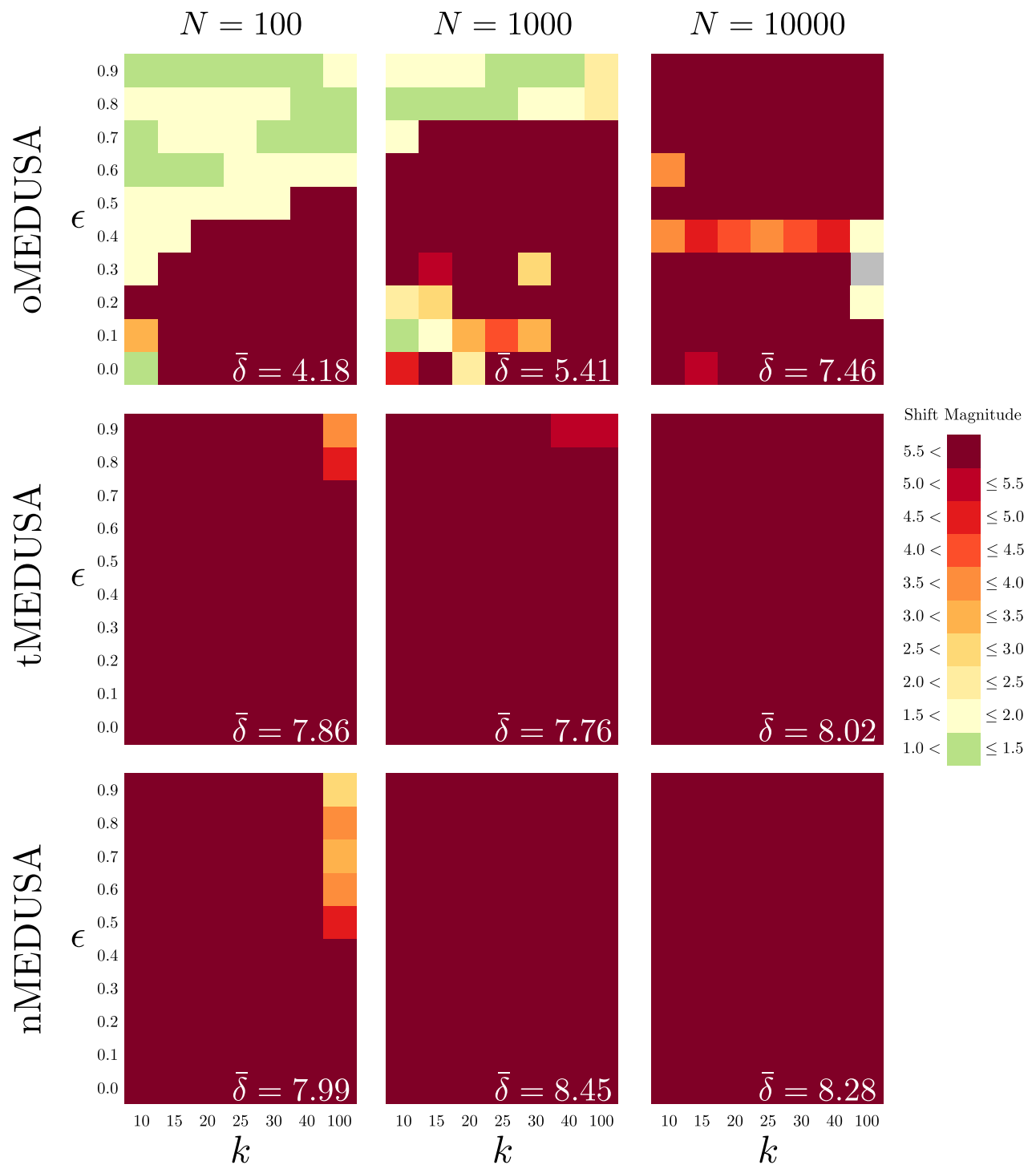

FIGURE S.14: 95\% quantile of shift magnitude in relative-extinction rate when a tworate model is selected. The three panels in each row summarize results for one algorithm (oMEDUSA, tMEDUSA, and nMEDUSA, from top to bottom), the three panels in each column summarize results for one tree size (with $N=100,1,000,10,000$ species, from left to right). Within each panel, we plot the number of terminal lineages, $k$, against the relative-extinction rate used in the simulation. Trees were simulated under a constant-rate birth-death process using the parameters summarized in Table S.18. The cells within each panel are colored to reflect the $95 \%$ quantile of the magnitude of the inferred shift in relative-extinction rate when a two-rate model is incorrectly identified (see Shift Magnitude legend). In order to accommodate the bimodal distribution of rate shifts, the magnitude of each relative-extinction-rate shift was computed as the ratio of the higher rate to the lower rate, regardless of which rate corresponded to the background-rate category. The average $95 \%$ quantile of the magnitude of shifts in relative-extinction rate shift is summarized in the lower right of each panel. 
bioRxiv preprint doi: https://doi.org/10.1101/011452; this version posted November 15,2014 . The copyright holder for this preprint (which was not certified by peer review) is the author/funder, who has granted bioRxiv a license to display the preprint in perpetuity. It is made available under aCC-BY-ND 4.0 International license.

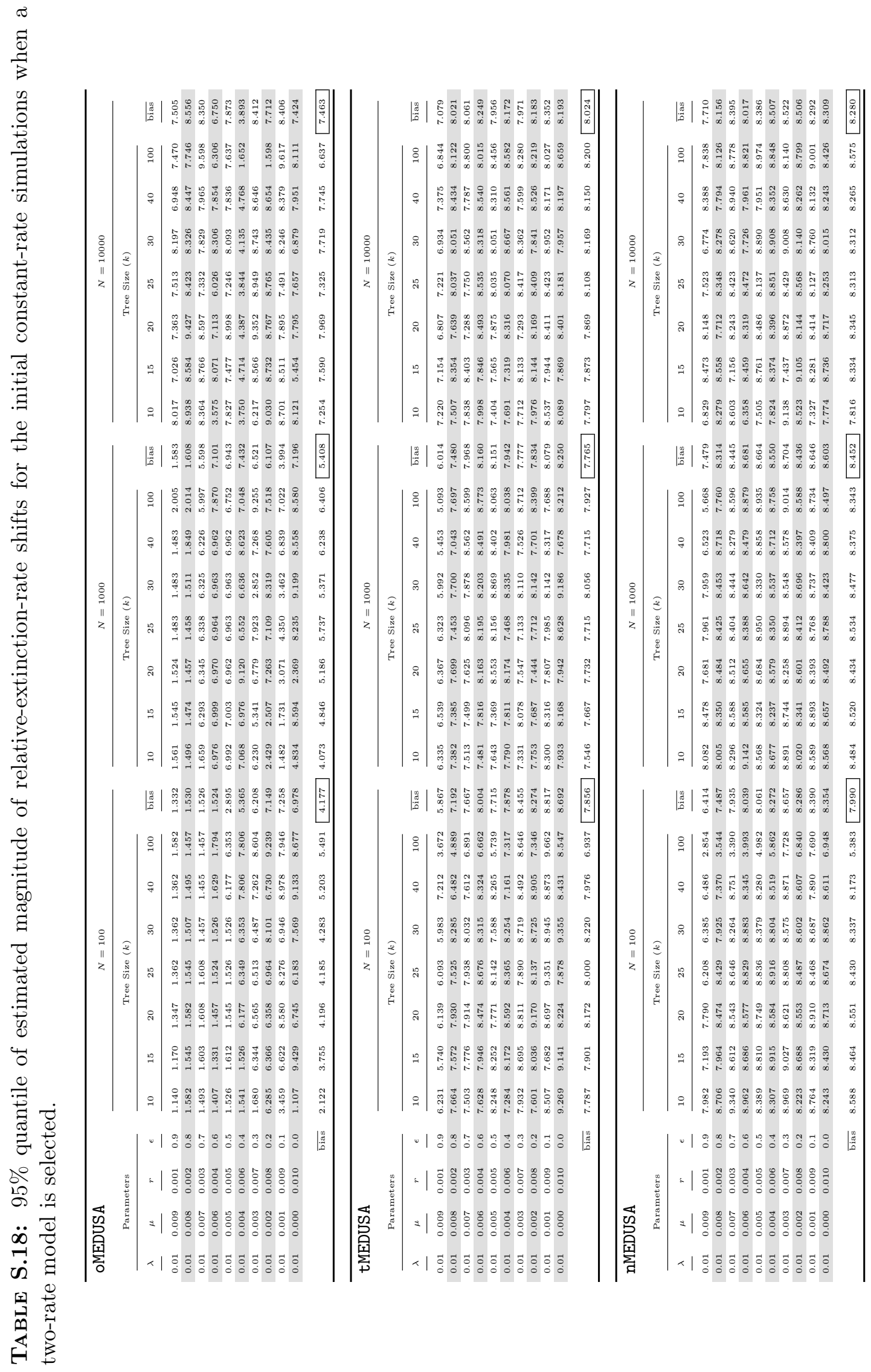




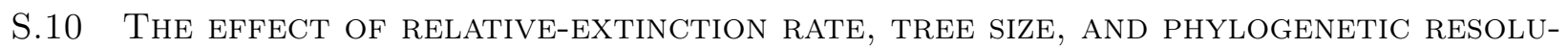
TION (CROWN SHIFTS).

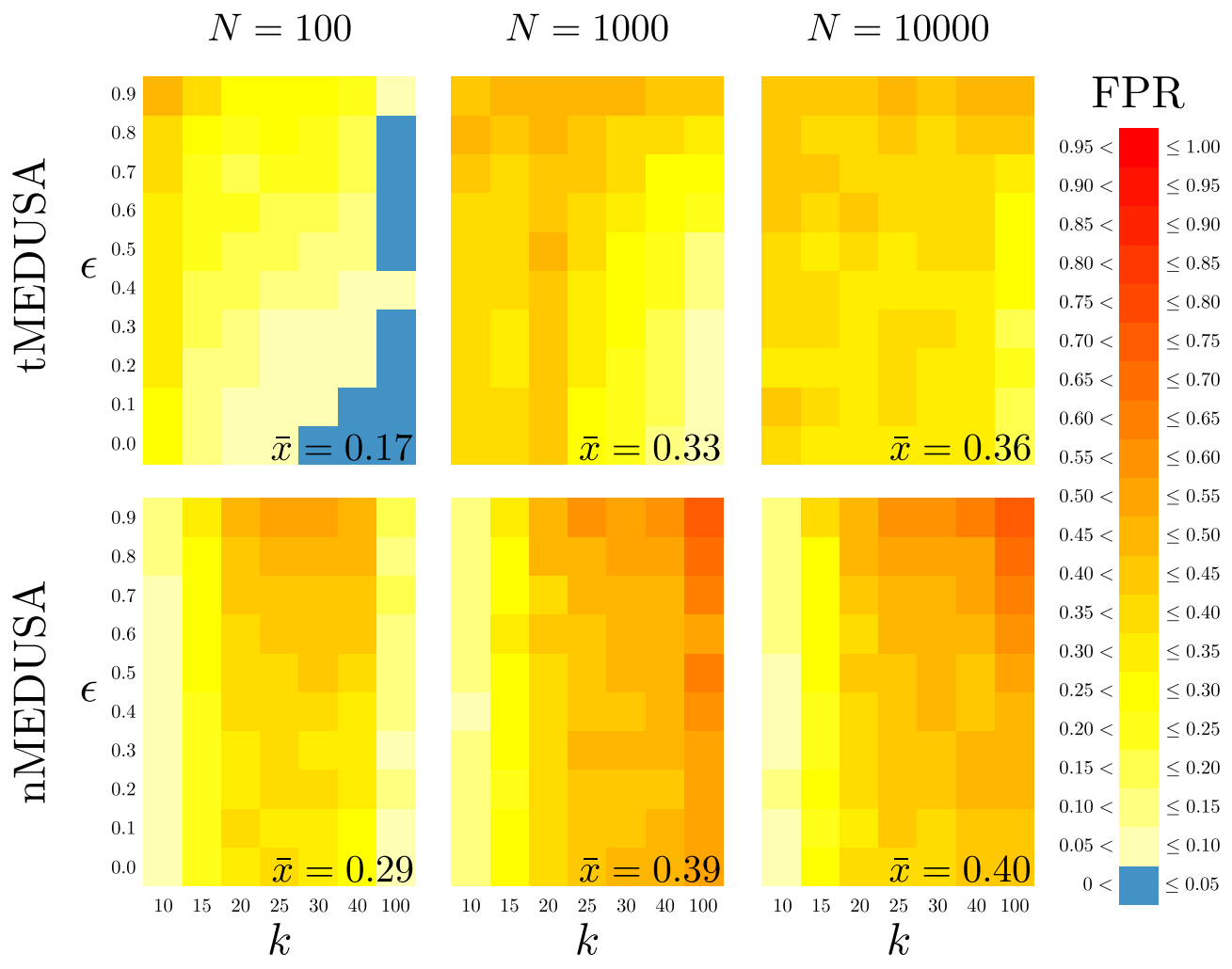

FIgURE S.15: Type I error rates for the three MEDUSA algorithms (crown shifts). The three panels in each row summarize results for one algorithm (oMEDUSA, tMEDUSA, and nMEDUSA, from top to bottom), the three panels in each column summarize results for one tree size (with $N=100,1,000,10,000$ species, from left to right). Within each panel, we plot the number of terminal lineages, $k$, against the relative-extinction rate used in the simulation. Trees were simulated under a constant-rate birth-death process using the parameters summarized in Table S.19. The cells within each panel are colored as a heat map reflecting the frequency with which spurious diversification-rate shifts were inferred (see legend). The average Type I error rate is summarized in the lower right of each panel. 
bioRxiv preprint doi: https://doi.org/10.1101/011452; this version posted November 15,2014 . The copyright holder for this preprint (which was not certified by peer review) is the author/funder, who has granted bioRxiv a license to display the preprint in perpetuity. It is made available under aCC-BY-ND 4.0 International license.

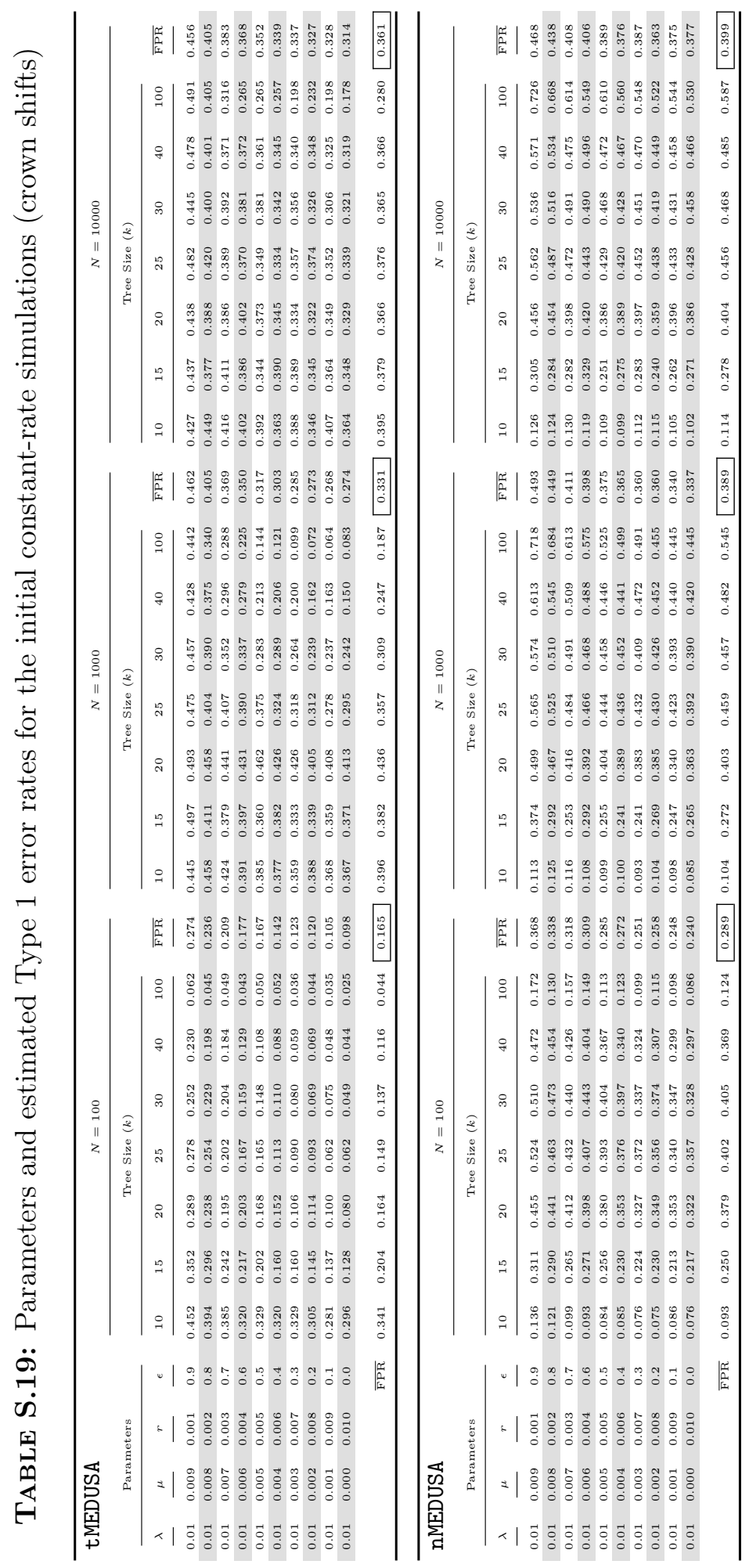

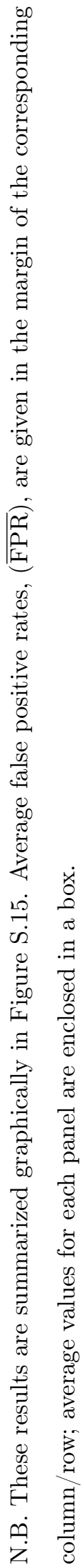




\section{S.11 Exploring the impact of Monte Carlo error on Type I error rates (Crown} SHIFTS)
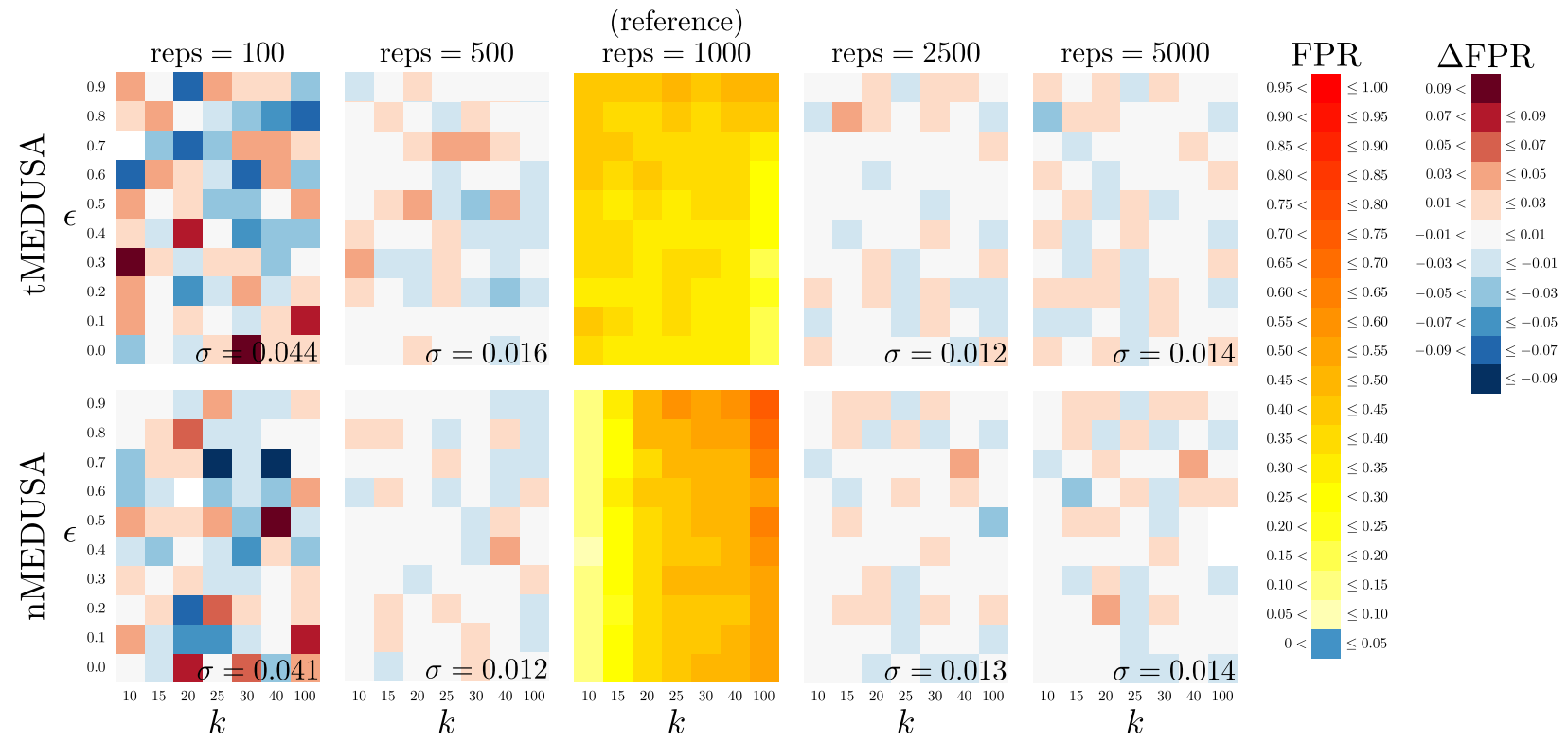

Figure S.16: Exploring the impact of Monte Carlo error on the complexity and intensity of estimated Type I error rates. The five panels in each row summarize results for one algorithm (tMEDUSA and nMEDUSA, from top to bottom), the three panels in each column summarize results based on the number of replicates (with 100 to 5,000 simulated trees, from left to right). The center column - which is reproduced from Figure 2 (the right-most column, with $N=10,000$ species)-summarizes the Type I error rates based on 1,000 simulated trees, where the cells of each panel are colored to reflect the frequency with which spurious diversification-rate shifts were inferred (see FPR legend). These results serve as a reference to explore the impact of the number of simulated replicates on the inferred patterns and overall rates of Type I error. In the other columns, the cells within each panel are colored to reflect the difference in the estimated Type I error rate relative to that of its corresponding reference cell (see $\Delta$ FPR legend). The standard deviation, $\sigma$, of the Type I error rate is summarized in the lower right of each panel. These experiments suggest that $\sim 500$ replicates are sufficient for precise estimates of Type I error rates. For detailed results, see Table $S .20$. 
bioRxiv preprint doi: https://doi.org/10.1101/011452; this version posted November 15, 2014. The copyright holder for this preprint (which was not certified by peer review) is the author/funder, who has granted bioRxiv a license to display the preprint in perpetuity. It is made available under aCC-BY-ND 4.0 International license.

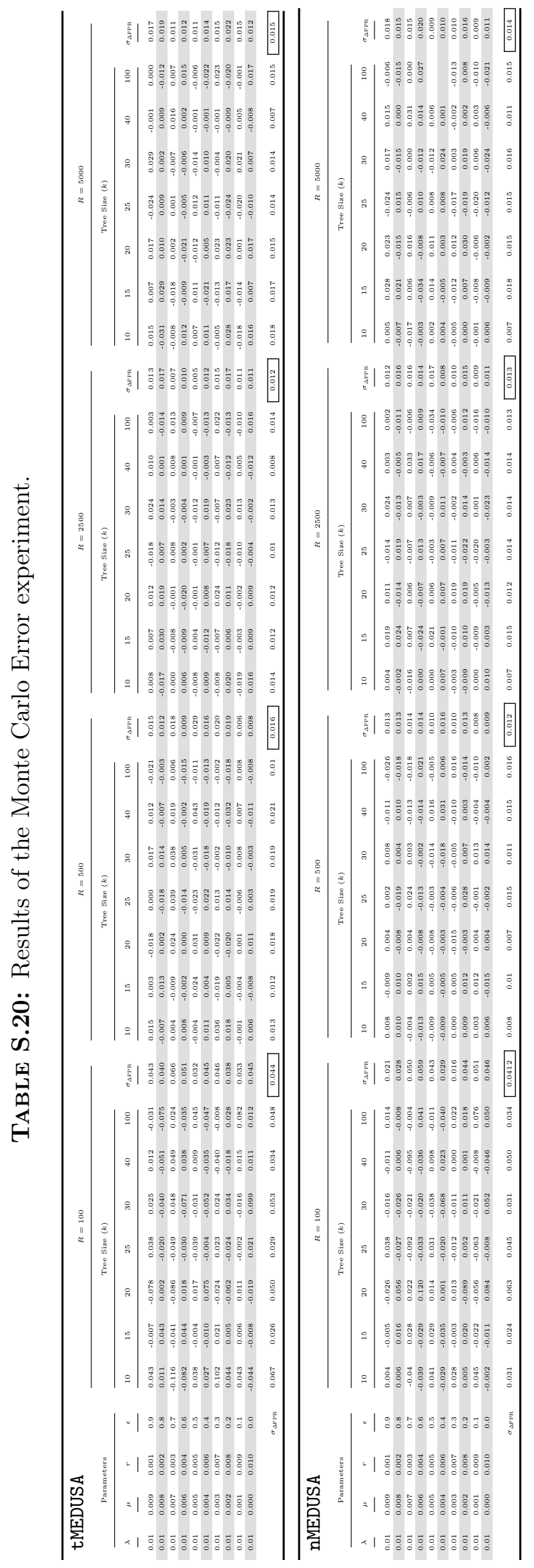




\section{S.12 The effect of absolute diversification Rates on Type I error (Crown shifts)}
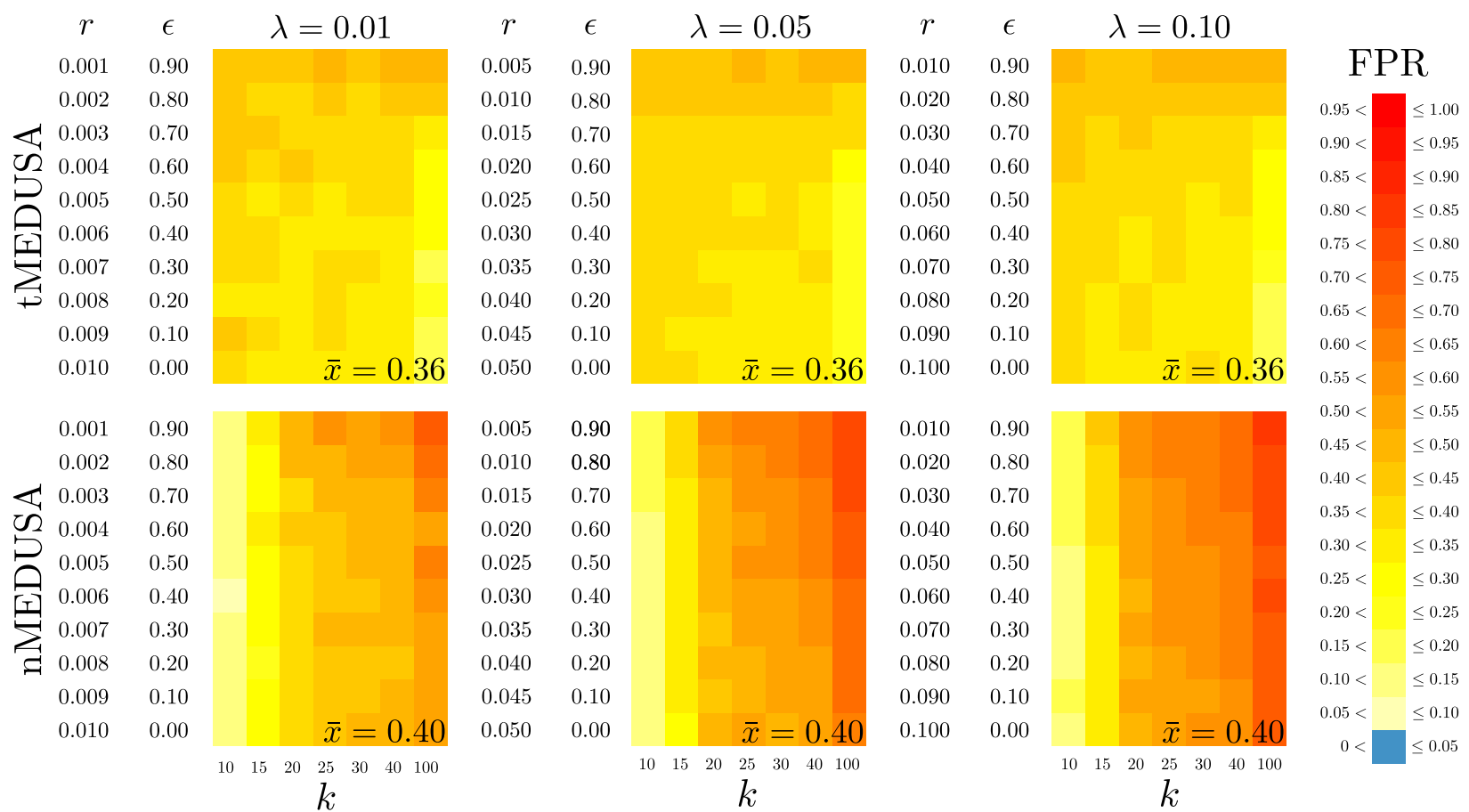

FIgURE S.17: The effect of increasing the absolute diversification rate on Type I error: maintaining consistent relative-extinction rates. The three panels in each row summarize results for one algorithm (tMEDUSA, and nMEDUSA from top to bottom), the panels in each column summarize results for one absolute speciation rate $(\lambda=0.01,0.05,0.10$, from left to right). Trees with $N=10,000$ species were simulated under a constant-rate birth-death process using the parameters described in Table S.21. For the absolute rates explored here, we specified a set of extinction rates, $\mu$, such that the set of relative-extinction rates, $\epsilon=\{0.00,0.01, \ldots, 0.90\}$ are consistent with those used in the initial simulation (Figure 2, reproduced here in the left column). Within each panel, we plot the number of terminal lineages, $k$, against the diversification rate, $r$. The cells within each panel are colored to reflect the frequency with which spurious diversification-rate shifts were inferred (see legend). The average Type I error rate is summarized in the lower right of each panel. 
bioRxiv preprint doi: https://doi.org/10.1101/011452; this version posted November 15, 2014. The copyright holder for this preprint (which was not certified by peer review) is the author/funder, who has granted bioRxiv a license to display the preprint in perpetuity. It is made available under aCC-BY-ND 4.0 International license.

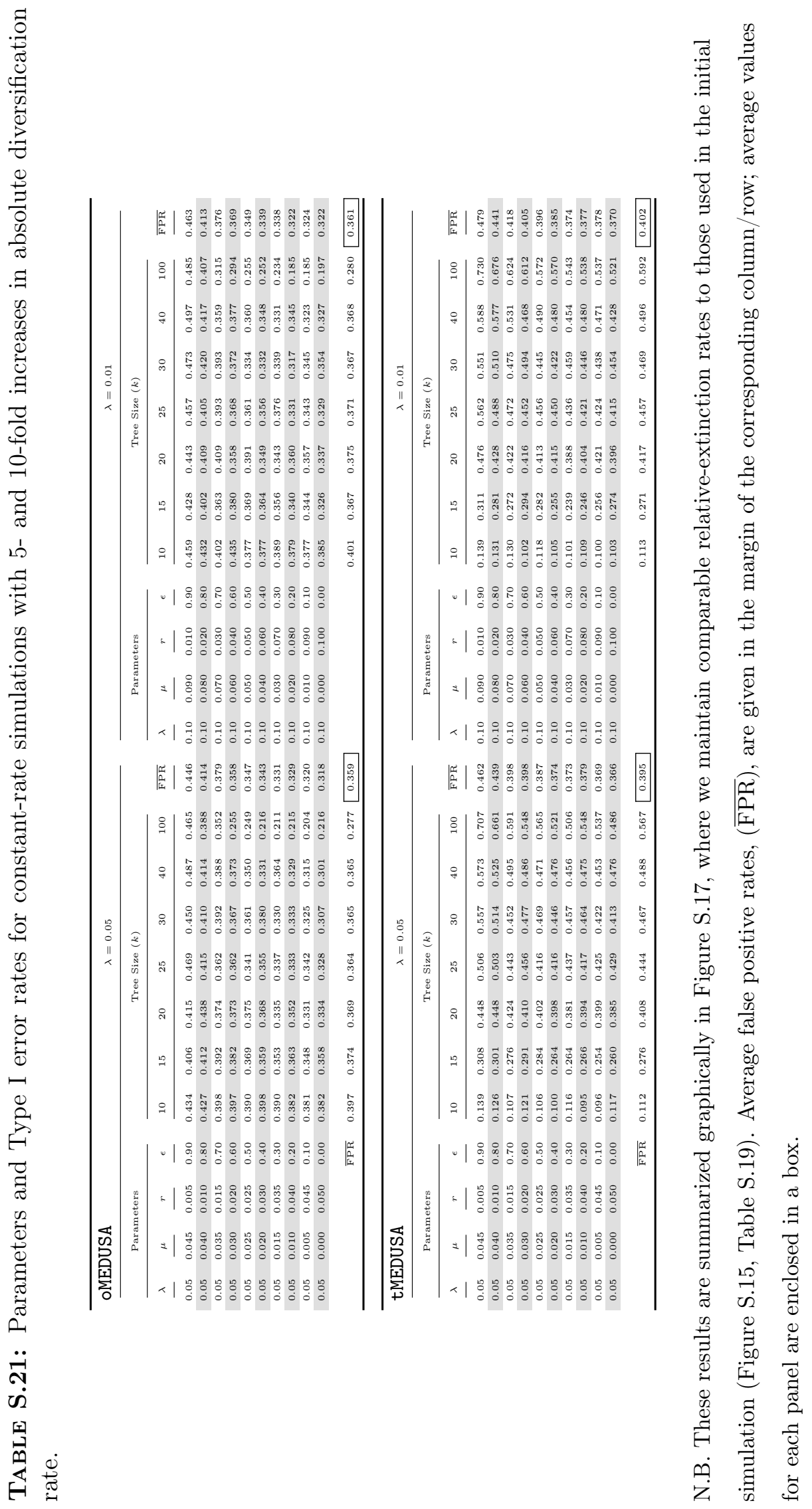




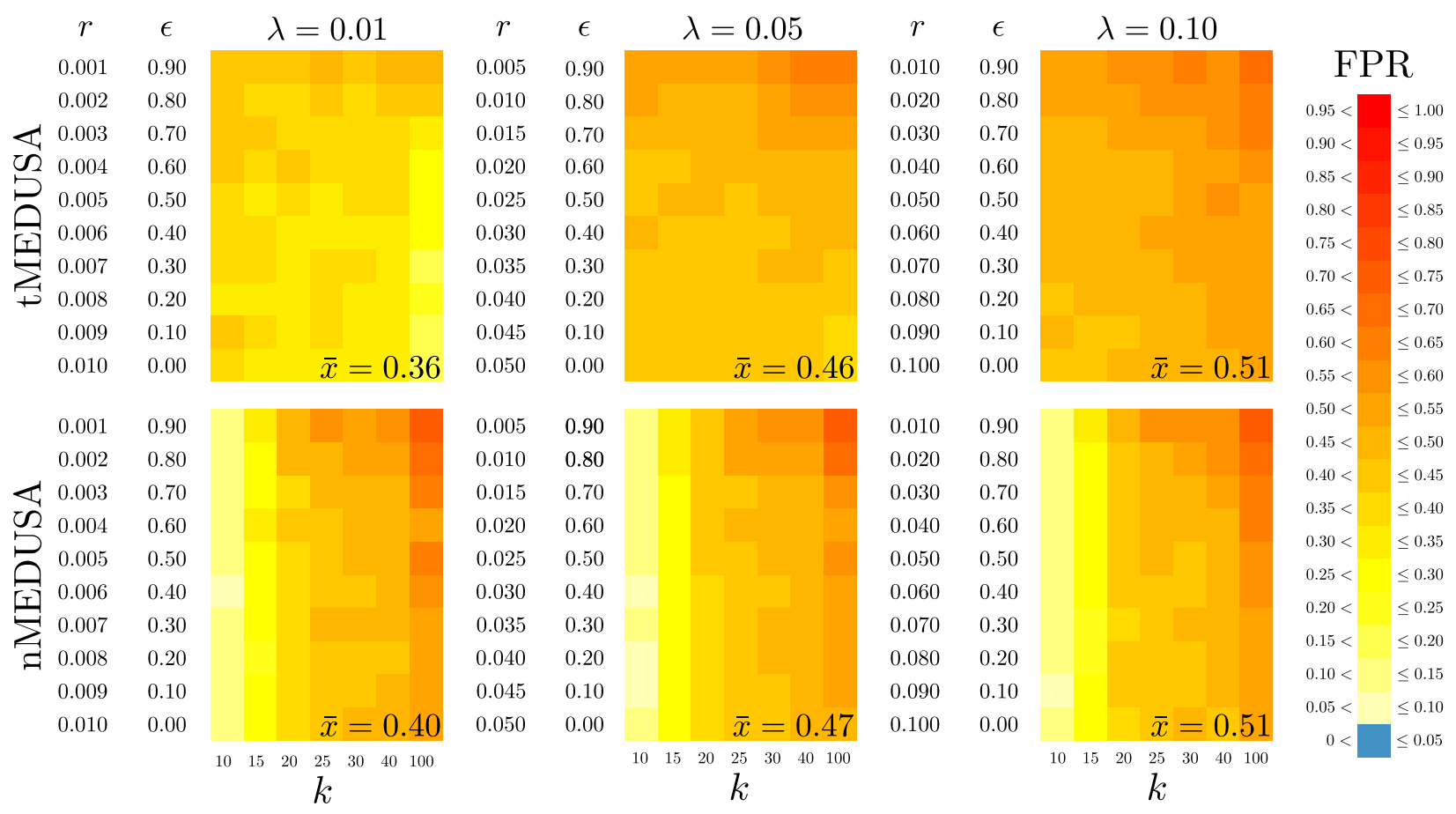

FiguRE S.18: The effect of increasing the absolute diversification rate on Type I error: maintaining consistent net-diversification rates. The three panels in each row summarize results for one algorithm (tMEDUSA, and nMEDUSA from top to bottom), the panels in each column summarize results for one absolute speciation rate $(\lambda=0.01,0.05,0.10$, from left to right). Trees with $N=10,000$ species were simulated under a constant-rate birth-death process using the parameters described in Table $\mathrm{S.22}$. For the absolute rates explored here, we specified a set of extinction rates, $\mu$, such that the set of net-diversification rates, $r=\{0.001,0.002, \ldots, 0.010\}$ are consistent with those used in the initial simulation (Figure 2, reproduced here in the left column). Within each panel, we plot the number of terminal lineages, $k$, against the diversification rate, $r$. The cells within each panel are colored to reflect the frequency with which spurious diversification-rate shifts were inferred (see legend). The average Type I error rate is summarized in the lower right of each panel. 
bioRxiv preprint doi: https://doi.org/10.1101/011452; this version posted November 15, 2014. The copyright holder for this preprint (which was not certified by peer review) is the author/funder, who has granted bioRxiv a license to display the preprint in perpetuity. It is made available under aCC-BY-ND 4.0 International license.

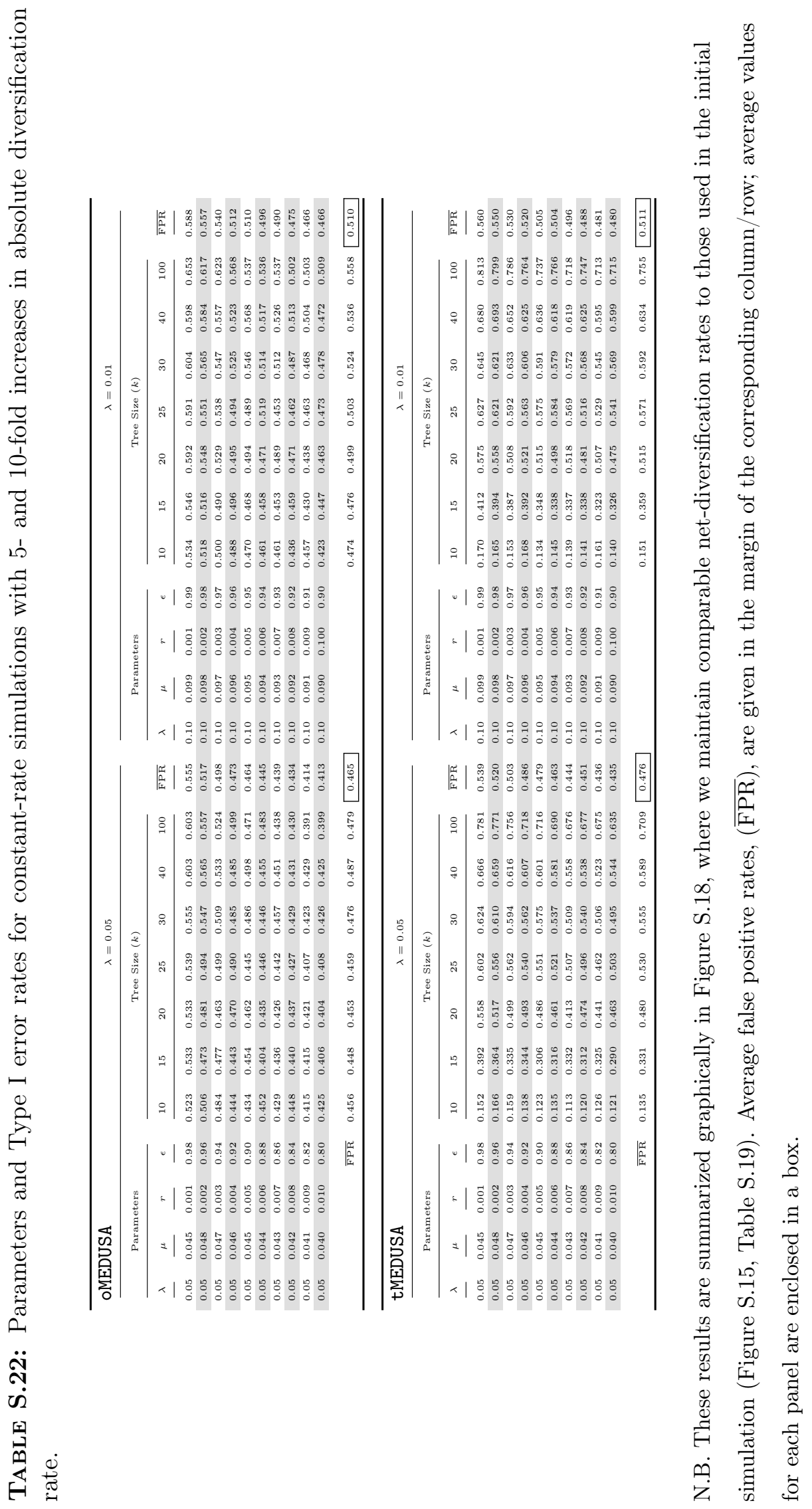




\section{S.13 Exploring threshold EFfects on Type I ERror Rates (CROWN Shifts)}

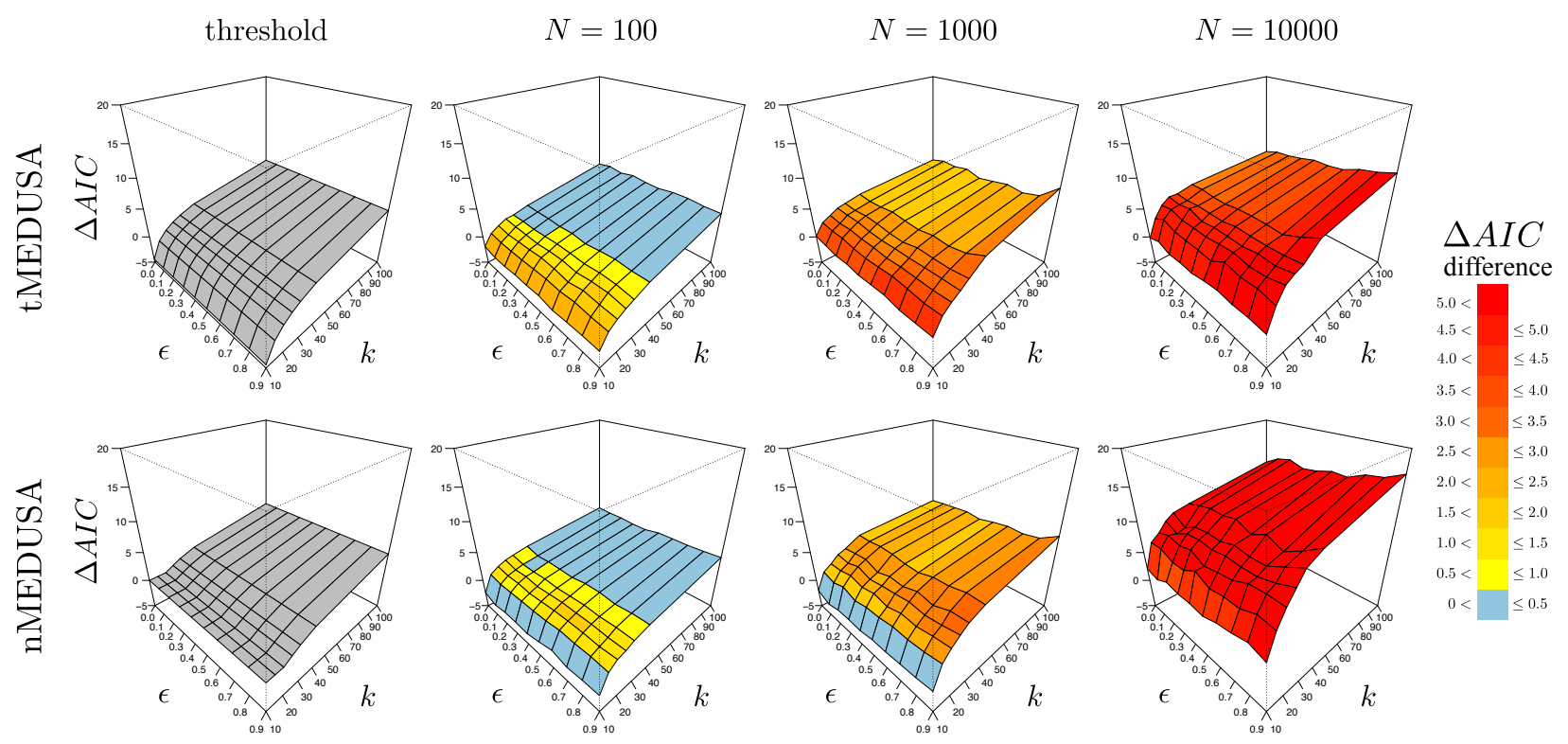

FIgURE S.19: Critical and computed $\triangle \mathrm{AIC}$ surfaces for two MEDUSA algorithms. The four panels in each row correspond to one algorithm (tMEDUSA and nMEDUSA, from top to bottom). The first column depicts the surface of $\Delta \mathrm{AIC}_{\text {crit }}$ threshold values for each algorithm; when the difference between the AIC scores of two competing models exceeds this threshold, the more complex model is selected. Columns $2-4$ summarize the $\Delta \mathrm{AIC}_{\text {crit }}$ values computed from simulated trees of each size $(N=100,1,000,10,000)$. The computed $\Delta$ AIC surfaces are colored to reflect the degree to which they exceed the corresponding $\Delta \mathrm{AIC}_{\text {crit }}$ threshold (see legend). Surfaces are computed from the trees used in the initial constant-rate simulation (c.f., Figure S.15) under parameters described in Table S.19. For detailed values, see Table S.23 
bioRxiv preprint doi: https://doi.org/10.1101/011452; this version posted November 15, 2014. The copyright holder for this preprint (which was not certified by peer review) is the author/funder, who has granted bioRxiv a license to display the preprint in perpetuity. It is made available under aCC-BY-ND 4.0 International license.






\section{S.14 Exploring Type I error RATES FOr COMPlete SPECies trees (CROWn Shifts)}

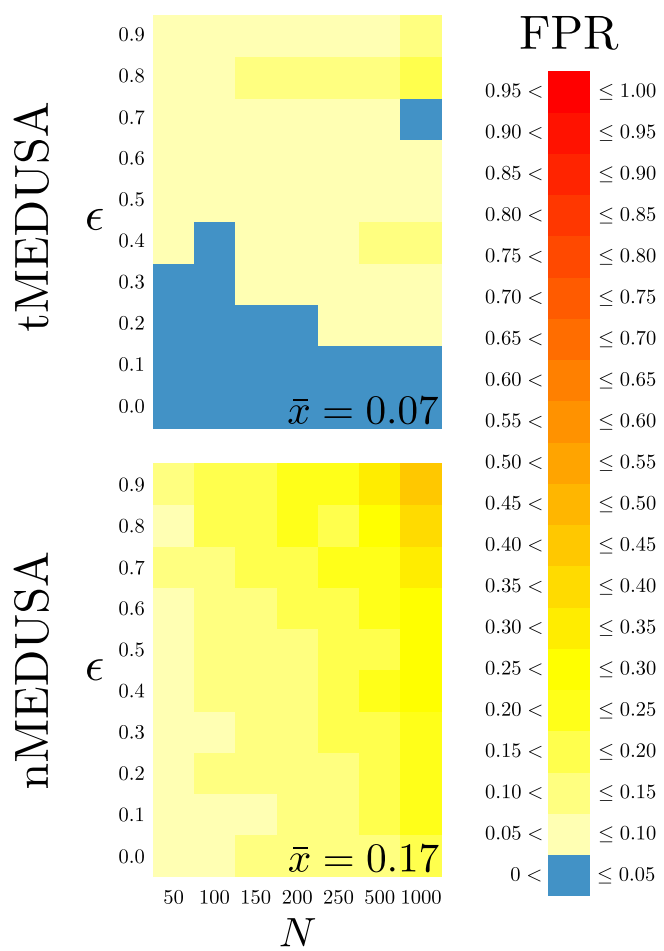

Figure S.20: Type I error rates for completely sampled trees. Each row summarizes results for one algorithm (tMEDUSA and nMEDUSA on the top and bottom, respectively). Within each panel, we plot the number of species in the tree, $N$, against the relative-extinction rate used in the simulation. Trees were simulated under a constant-rate birth-death process using the parameters summarized in Table S.24. The cells within each panel are colored as a heat map reflecting the frequency with which spurious diversification-rate shifts were inferred (see FPR legend). The average Type I error rate is summarized in the lower right of each panel. 
bioRxiv preprint doi: https://doi.org/10.1101/011452; this version posted November 15, 2014. The copyright holder for this preprint (which was not certified by peer review) is the author/funder, who has granted bioRxiv a license to display the preprint in perpetuity. It is made available under aCC-BY-ND 4.0 International license.

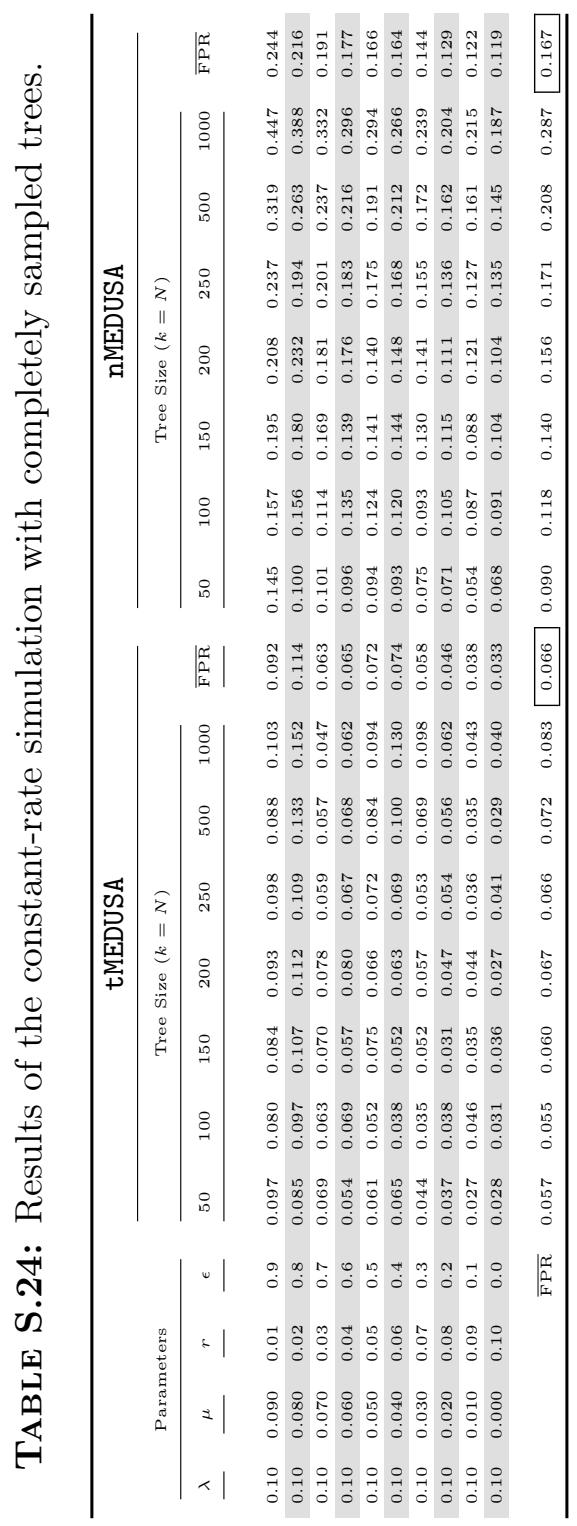




\section{S.15 Parameter estimation (CROWn Shifts)}
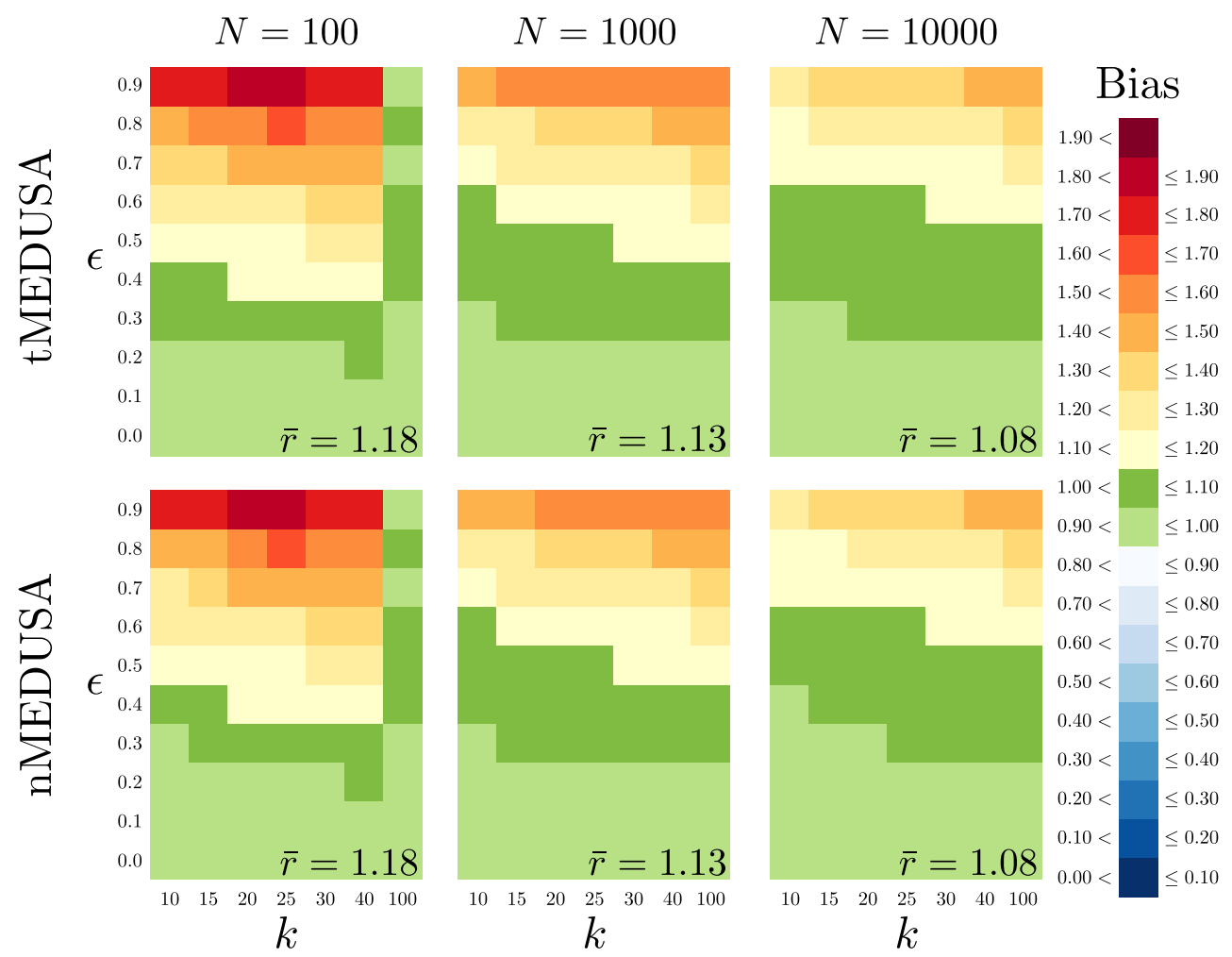

FIGURE S.21: Bias in estimated net-diversification rate when a single-rate model is selected. The three panels in each row summarize results for one algorithm (tMEDUSA and nMEDUSA, from top to bottom), the three panels in each column summarize results for one tree size (with $N=100,1,000,10,000$ species, from left to right). Within each panel, we plot the number of terminal lineages, $k$, against the relative-extinction rate used in the simulation. Trees were simulated under a constant-rate birth-death process using the parameters summarized in Table S.25. The cells within each panel are colored to reflect the average difference between the estimated and true netdiversification rates when a one-rate model is correctly identified (see Bias legend). The average bias in estimates of relative extinction-rate is summarized in the lower right of each panel. 
bioRxiv preprint doi: https://doi.org/10.1101/011452; this version posted November 15, 2014. The copyright holder for this preprint (which was not certified by peer review) is the author/funder, who has granted bioRxiv a license to display the preprint in perpetuity. It is made available under aCC-BY-ND 4.0 International license.

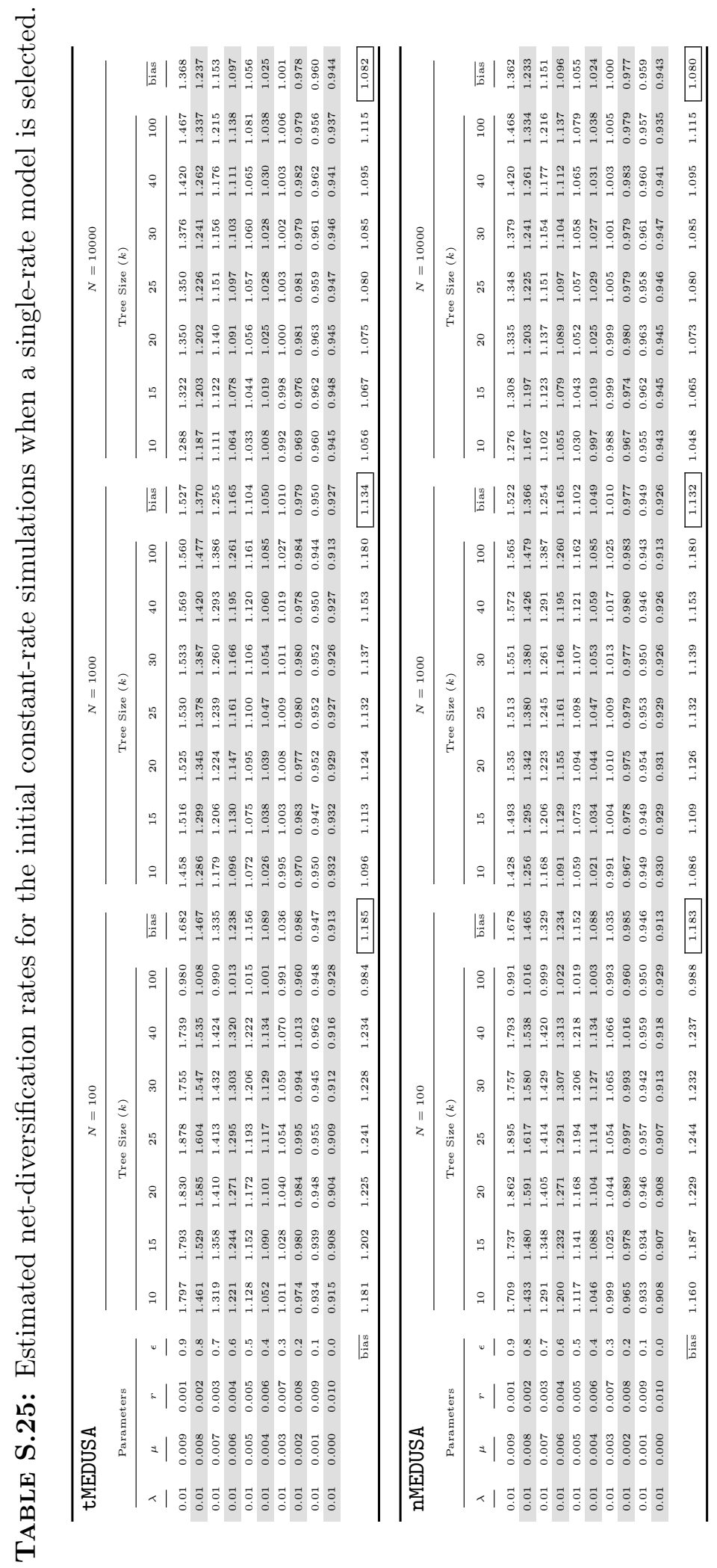



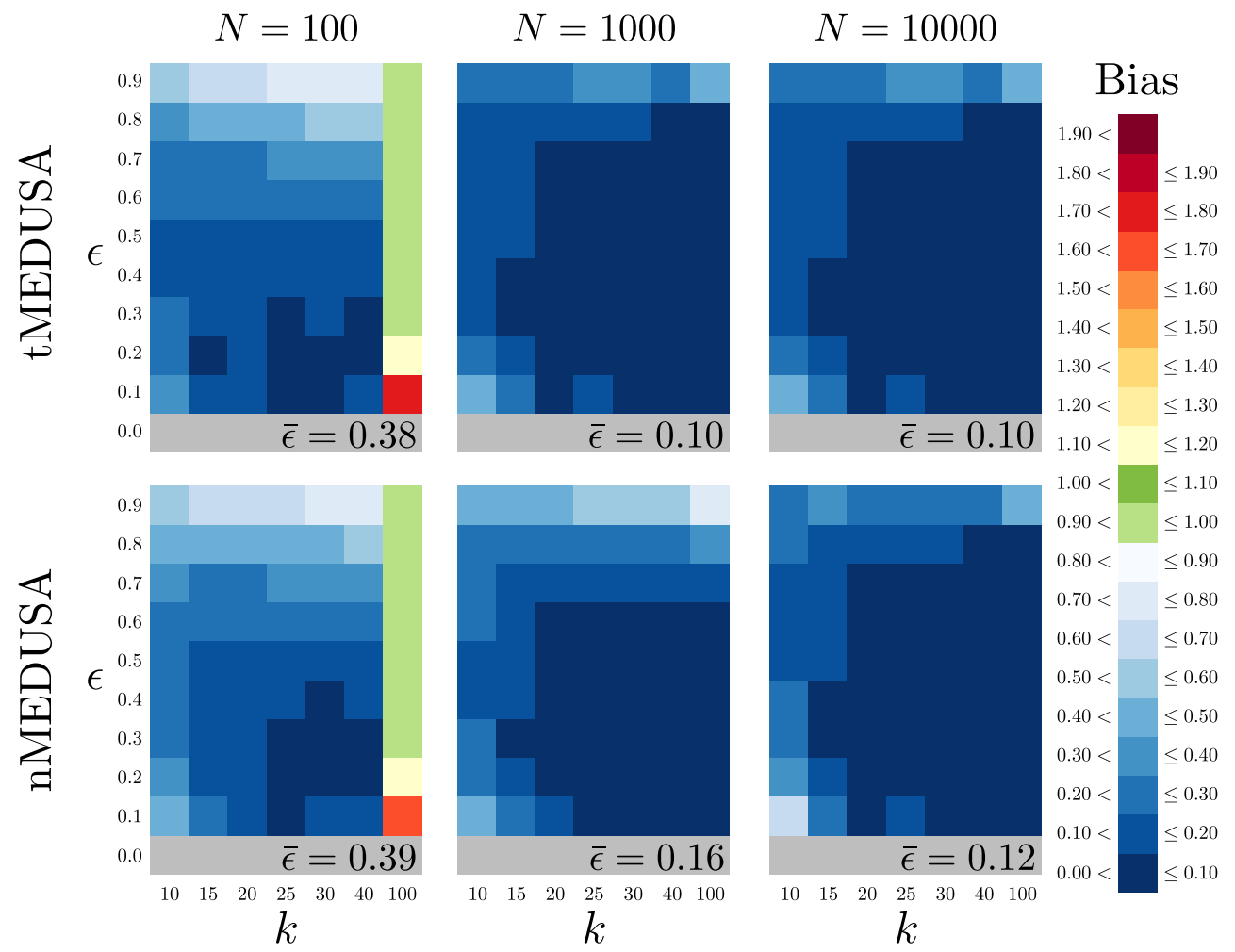

FIgURE S.22: Bias in estimated relative-extinction rate when a single-rate model is selected. The three panels in each row summarize results for one algorithm (tMEDUSA and nMEDUSA, from top to bottom), the three panels in each column summarize results for one tree size (with $N=100,1,000,10,000$ species, from left to right). Within each panel, we plot the number of terminal lineages, $k$, against the relative-extinction rate used in the simulation. Trees were simulated under a constant-rate birth-death process using the parameters summarized in Table S.12. The cells within each panel are colored to reflect the average difference between the estimated and true relative-extinction rates when a one-rate model is correctly identified (see Bias legend). The average bias in estimates of relative extinction-rate is summarized in the lower right of each panel. 
bioRxiv preprint doi: https://doi.org/10.1101/011452; this version posted November 15, 2014. The copyright holder for this preprint (which was not certified by peer review) is the author/funder, who has granted bioRxiv a license to display the preprint in perpetuity. It is made available under aCC-BY-ND 4.0 International license.
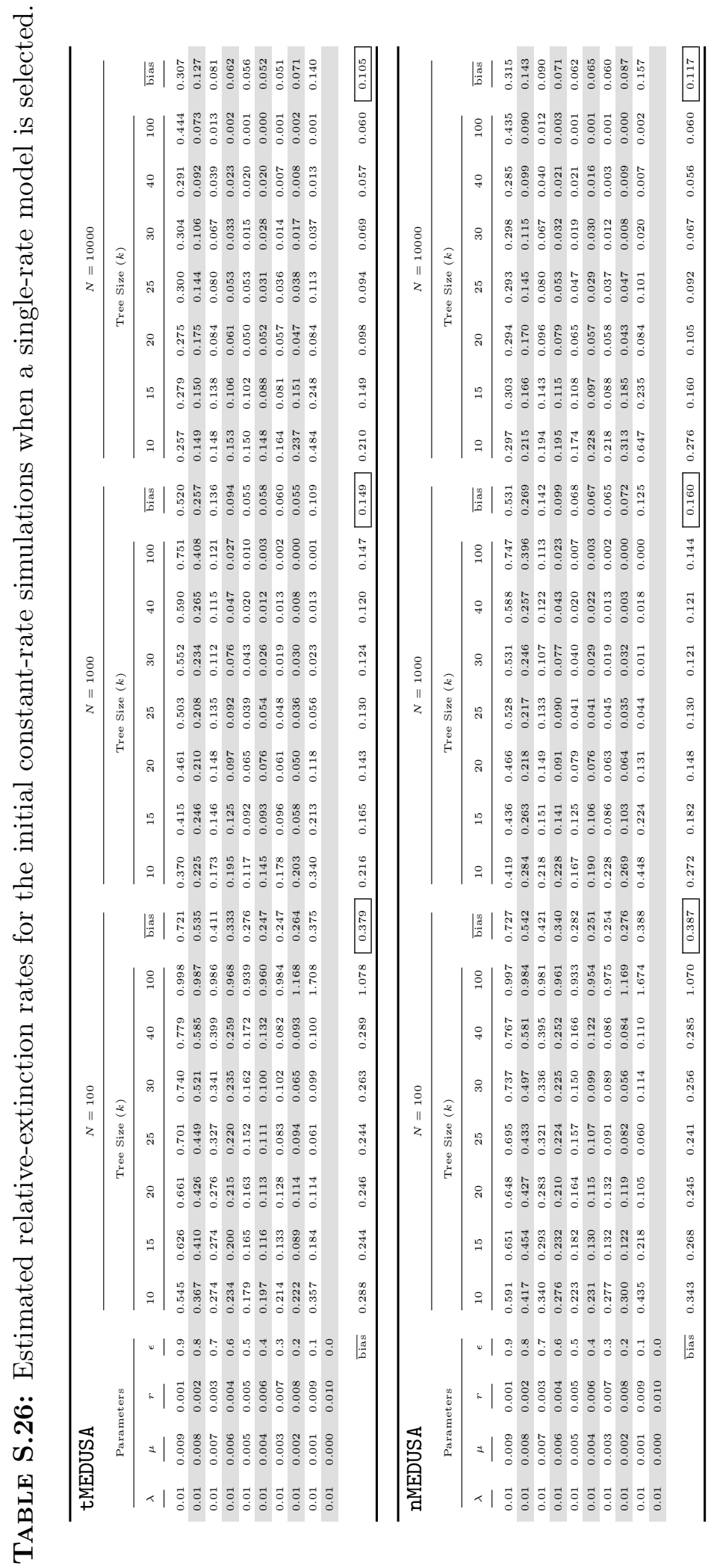

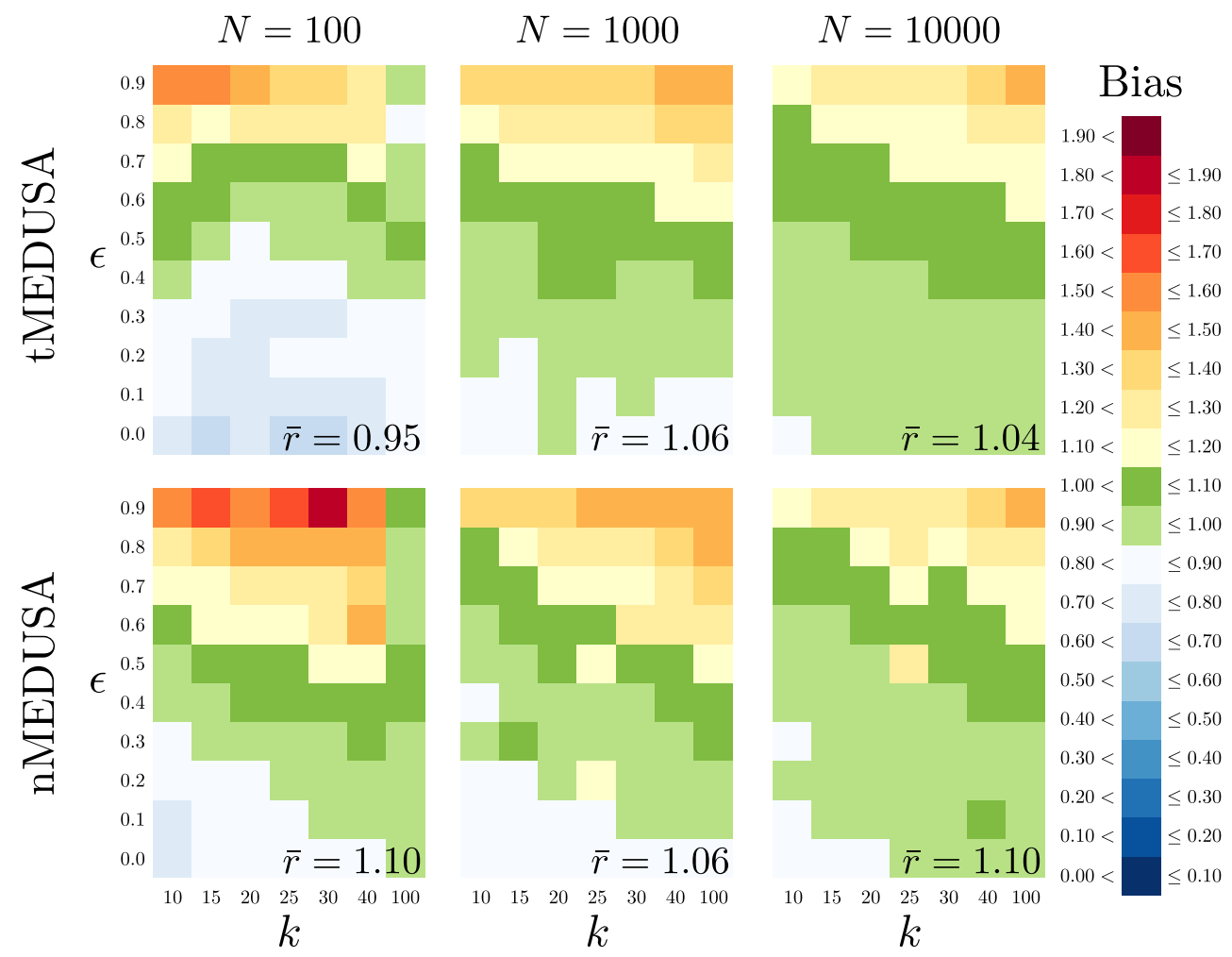

Figure S.23: Bias in estimated background net-diversification rates when a tworate model is selected. The three panels in each row summarize results for one algorithm (texttttMEDUSA, and nMEDUSA, from top to bottom), the three panels in each column summarize results for one tree size (with $N=100,1,000,10,000$ species, from left to right). Within each panel, we plot the number of terminal lineages, $k$, against the relative-extinction rate used in the simulation. Trees were simulated under a constant-rate birth-death process using the parameters summarized in Table S.27. The cells within each panel are colored to reflect the average difference between the estimated and true background net-diversification rate when a two-rate model is incorrectly identified (see Bias legend). The average bias in the inferred background net-diversification rate is summarized in the lower right of each panel. 
bioRxiv preprint doi: https://doi.org/10.1101/011452; this version posted November 15, 2014. The copyright holder for this preprint (which was not certified by peer review) is the author/funder, who has granted bioRxiv a license to display the preprint in perpetuity. It is made available under aCC-BY-ND 4.0 International license.

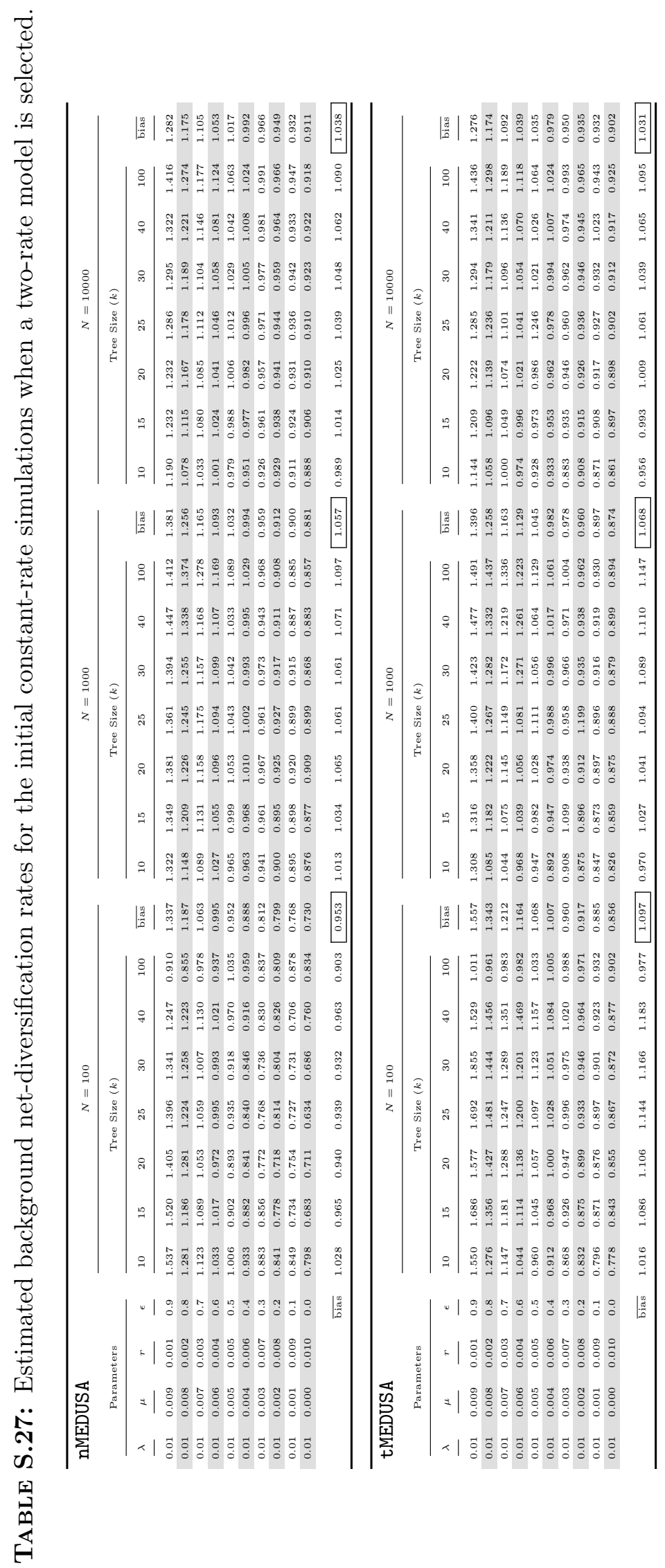



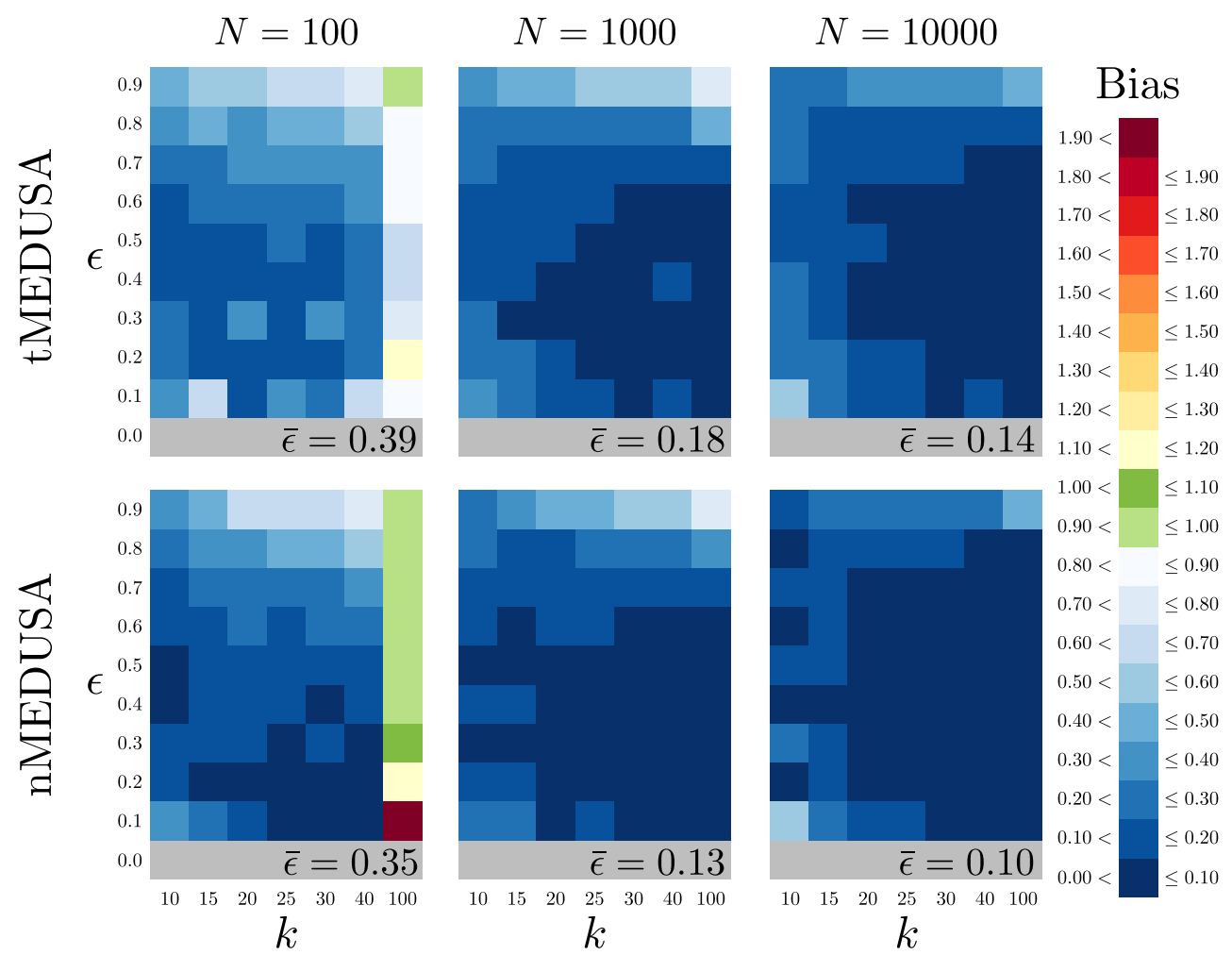

FIGURE S.24: Bias in estimated background relative-extinction rates when a two-rate model is selected. The three panels in each row summarize results for one algorithm (tMEDUSA, and nMEDUSA, from top to bottom), the three panels in each column summarize results for one tree size (with $N=100,1,000,10,000$ species, from left to right). Within each panel, we plot the number of terminal lineages, $k$, against the relative-extinction rate used in the simulation. Trees were simulated under a constant-rate birth-death process using the parameters summarized in Table S.28. The cells within each panel are colored to reflect the average difference between the estimated and true background relative-extinction rate when a two-rate model is incorrectly identified (see Bias legend). The average bias in the inferred background relative-extinction rate is summarized in the lower right of each panel. 
bioRxiv preprint doi: https://doi.org/10.1101/011452; this version posted November 15, 2014. The copyright holder for this preprint (which was not certified by peer review) is the author/funder, who has granted bioRxiv a license to display the preprint in perpetuity. It is made available under aCC-BY-ND 4.0 International license.

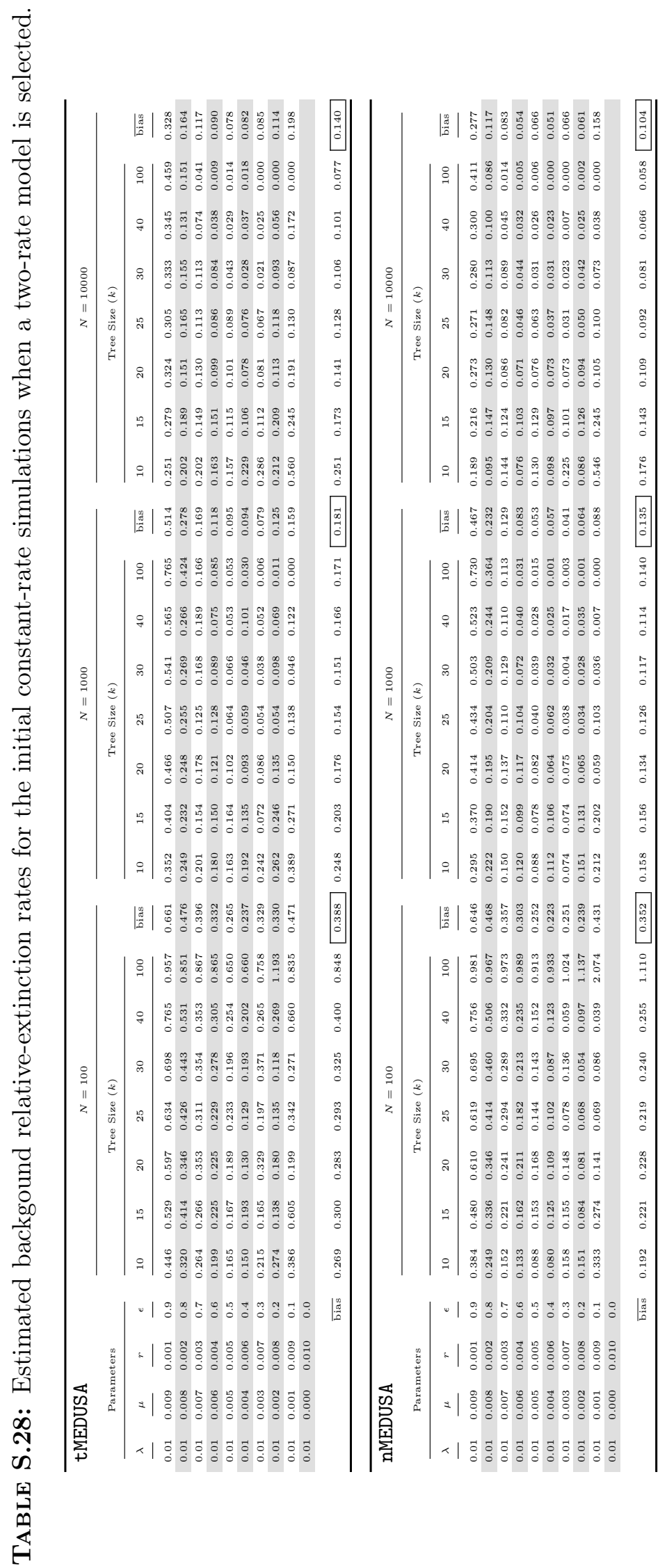



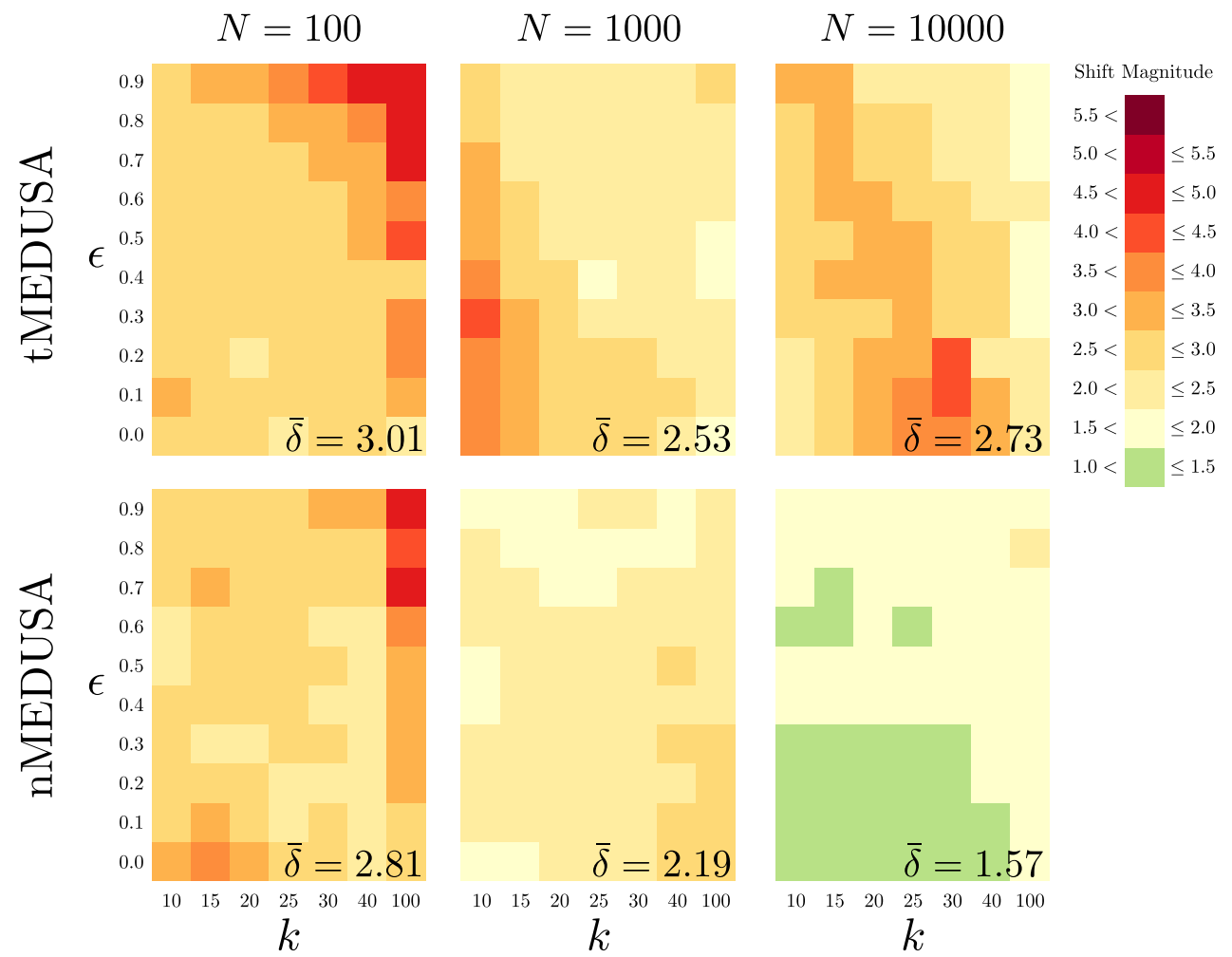

Figure S.25: Magnitude of shifts in net-diversification rate when a two-rate model is chosen. The three panels in each row summarize results for one algorithm (tMEDUSA, and nMEDUSA, from top to bottom), the three panels in each column summarize results for one tree size (with $N=100,1,000,10,000$ species, from left to right). Within each panel, we plot the number of terminal lineages, $k$, against the relative-extinction rate used in the simulation. Trees were simulated under a constant-rate birth-death process using the parameters summarized in Table S.29. The cells within each panel are colored as a heat map reflecting the average magnitude of shifts in net-diversification rate when a two-rate model is incorrectly identified (see Shift Magnitude legend). In order to accommodate the bimodal distribution of rate shifts, the magnitude of each netdiversification-rate shift was computed as the ratio of the higher rate to the lower rate, regardless of which rate corresponded to the background-rate category. The average magnitude of inferred diversification-rate shifts is summarized in the lower right of each panel. 
bioRxiv preprint doi: https://doi.org/10.1101/011452; this version posted November 15, 2014. The copyright holder for this preprint (which was not certified by peer review) is the author/funder, who has granted bioRxiv a license to display the preprint in perpetuity. It is made available under aCC-BY-ND 4.0 International license.

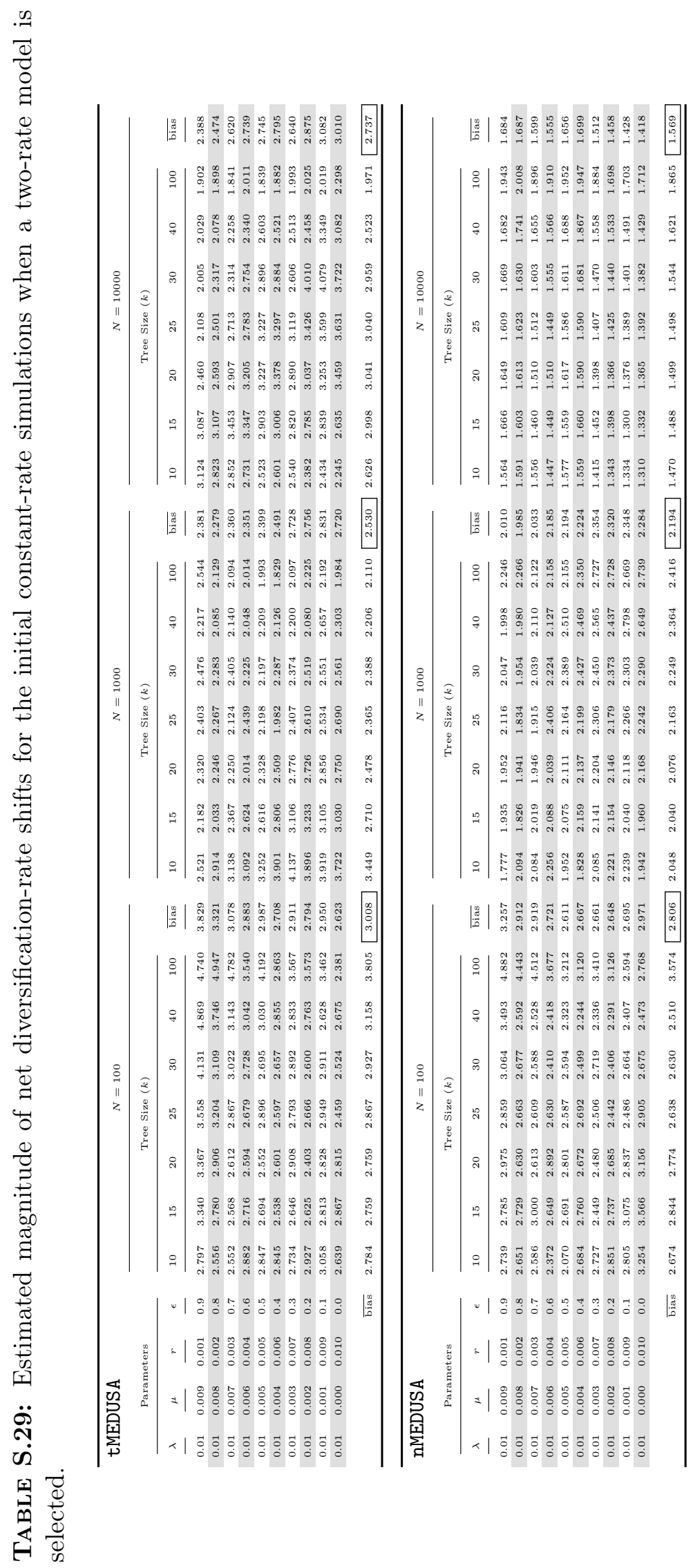



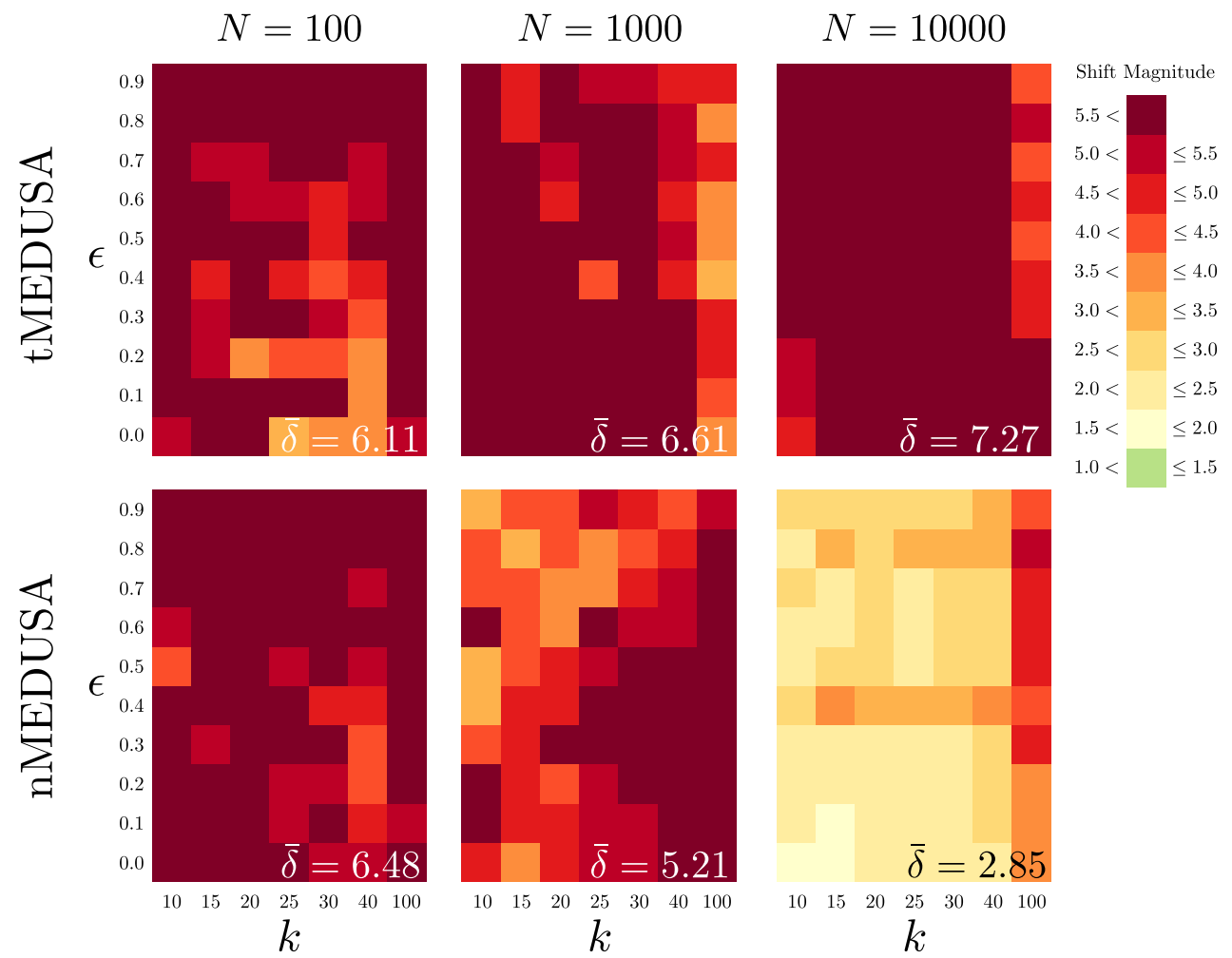

Figure S.26: 95\% quantile of shift magnitude in net-diversification rate when a tworate model is selected. The three panels in each row summarize results for one algorithm (tMEDUSA and nMEDUSA, from top to bottom), the three panels in each column summarize results for one tree size (with $N=100,1,000,10,000$ species, from left to right). Within each panel, we plot the number of terminal lineages, $k$, against the relative-extinction rate used in the simulation. Trees were simulated under a constant-rate birth-death process using the parameters summarized in Table S.30. The cells within each panel are colored to reflect the $95 \%$ quantile of the magnitude of the inferred shift in net-diversification rate when a two-rate model is incorrectly identified (see Shift Magnitude legend). In order to accommodate the bimodal distribution of rate shifts, the magnitude of each net-diversification-rate shift was computed as the ratio of the higher rate to the lower rate, regardless of which rate corresponded to the background-rate category. The average $95 \%$ quantile of the magnitude of shifts in net-diversification rate shift is summarized in the lower right of each panel. 
bioRxiv preprint doi: https://doi.org/10.1101/011452; this version posted November 15, 2014. The copyright holder for this preprint (which was not certified by peer review) is the author/funder, who has granted bioRxiv a license to display the preprint in perpetuity. It is made available under aCC-BY-ND 4.0 International license.

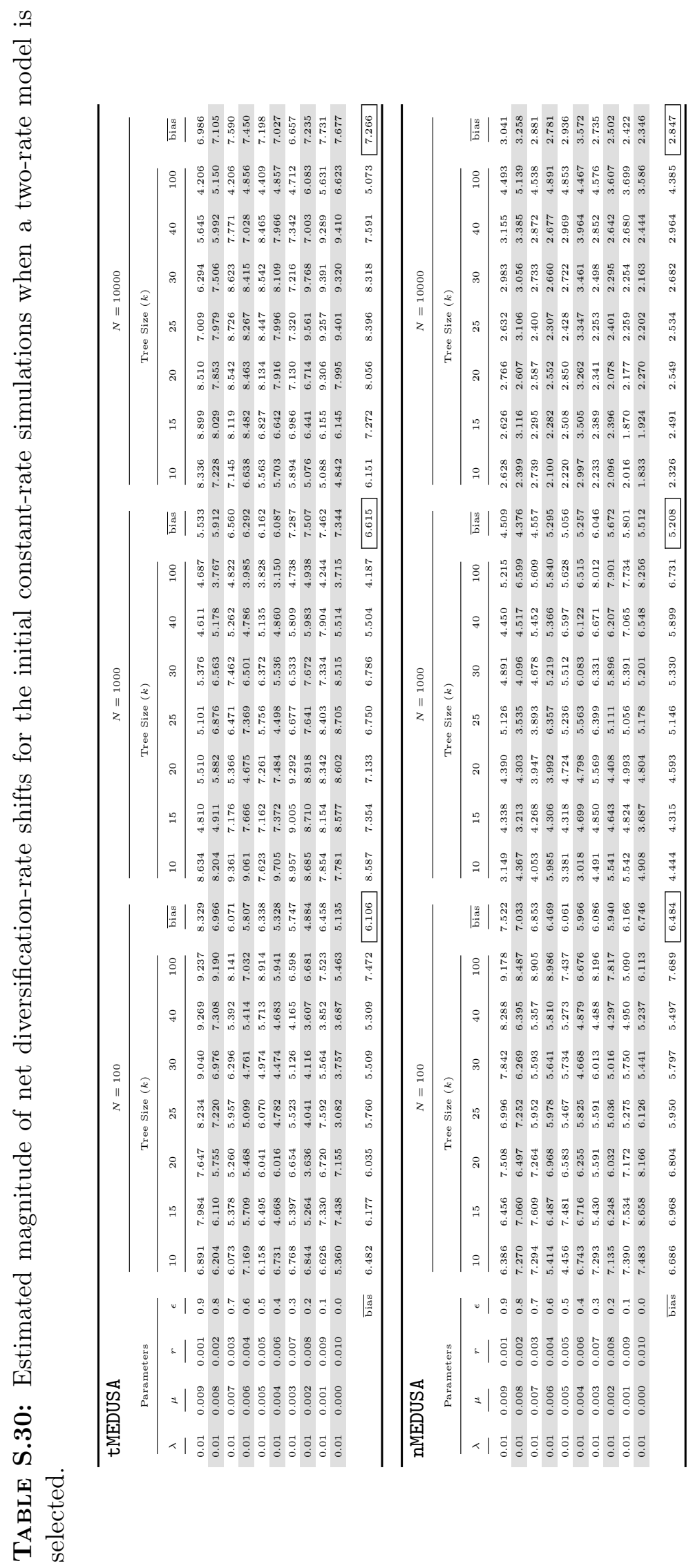



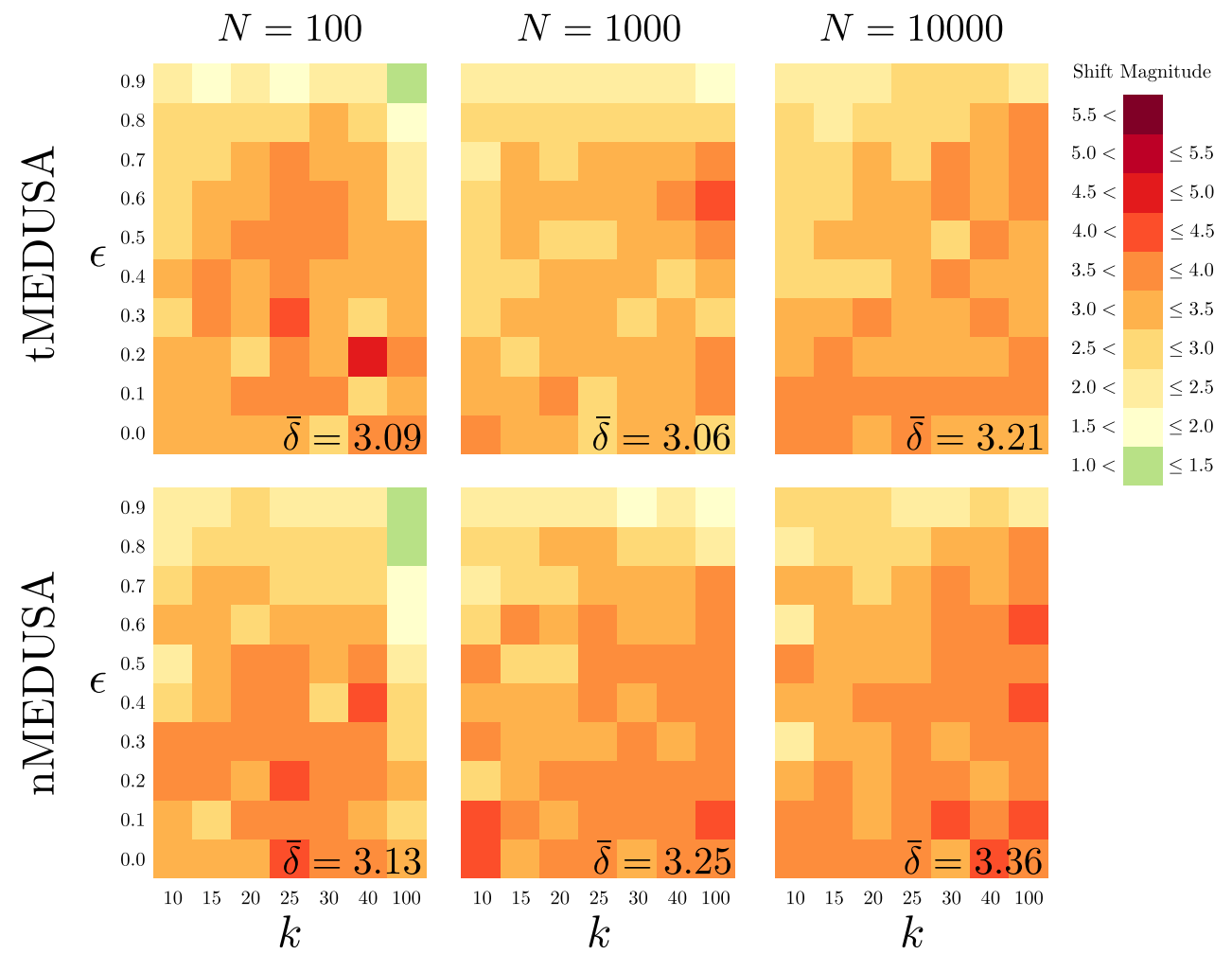

Figure S.27: Magnitude of shift in relative-extinction rate when a two-rate model is selected. The three panels in each row summarize results for one algorithm (tMEDUSA, and nMEDUSA, from top to bottom), the three panels in each column summarize results for one tree size (with $N=100,1,000,10,000$ species, from left to right). Within each panel, we plot the number of terminal lineages, $k$, against the relative-extinction rate used in the simulation. Trees were simulated under a constant-rate birth-death process using the parameters summarized in Table S.31. The cells within each panel are colored to reflect the average magnitude of the inferred shift in relative-extinction rate when a two-rate model is incorrectly identified (see Shift Magnitude legend). In order to accommodate the bimodal distribution of rate shifts, the magnitude of each relativeextinction-rate shift was computed as the ratio of the higher rate to the lower rate, regardless of which rate corresponded to the background-rate category. The average magnitude of shifts in relative-extinction rate shift is summarized in the lower right of each panel. 
bioRxiv preprint doi: https://doi.org/10.1101/011452; this version posted November 15, 2014. The copyright holder for this preprint (which was not certified by peer review) is the author/funder, who has granted bioRxiv a license to display the preprint in perpetuity. It is made available under aCC-BY-ND 4.0 International license.

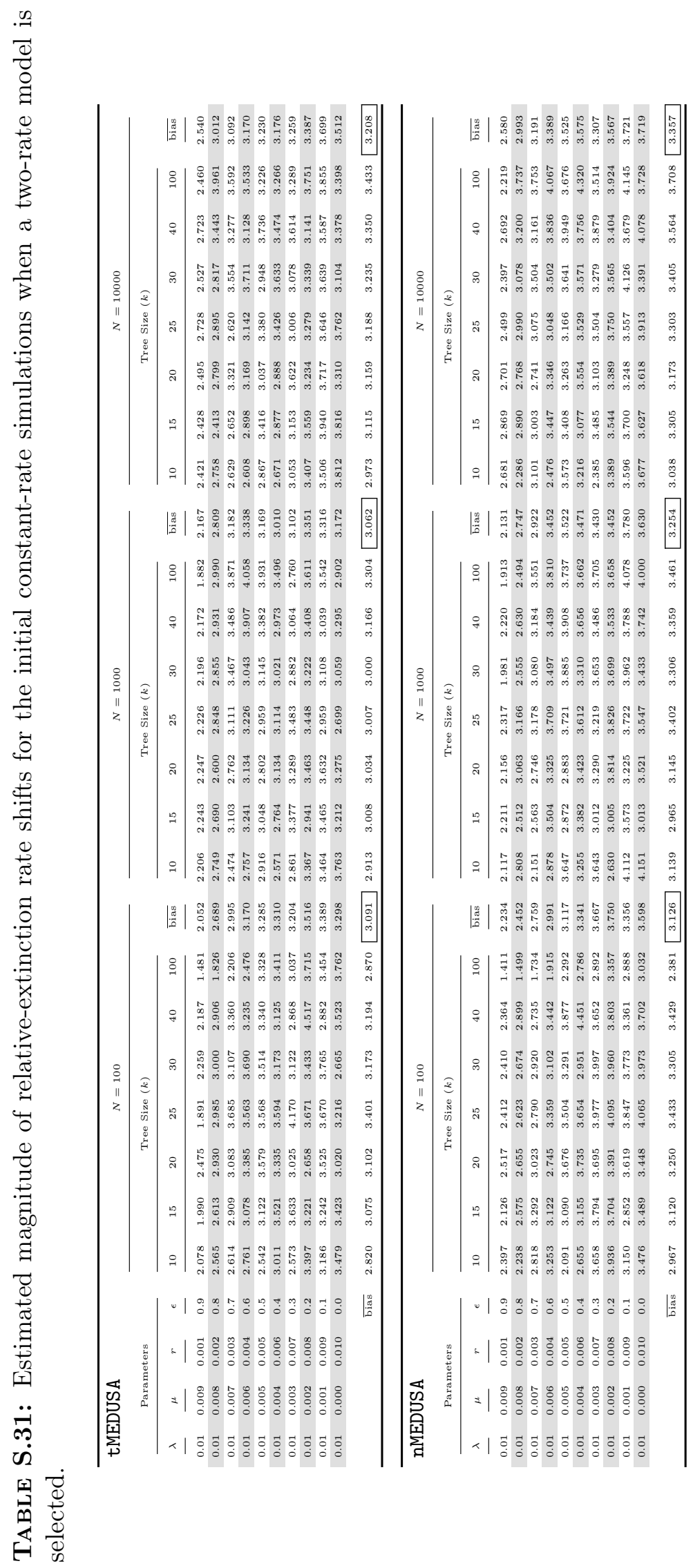



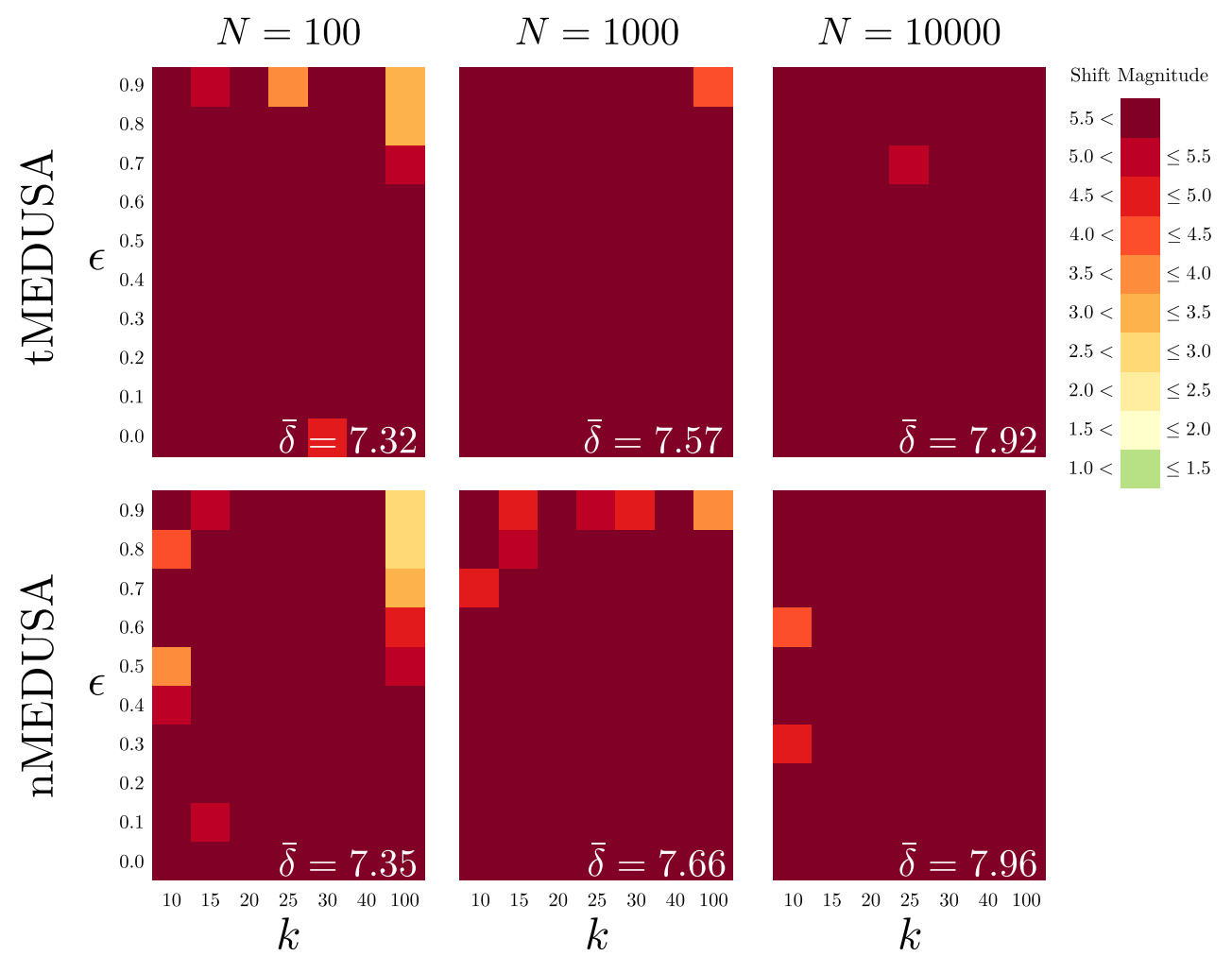

FIGURE S.28: 95\% quantile of shift magnitude in relative-extinction rate when a tworate model is selected. The three panels in each row summarize results for one algorithm (tMEDUSA and nMEDUSA, from top to bottom), the three panels in each column summarize results for one tree size (with $N=100,1,000,10,000$ species, from left to right). Within each panel, we plot the number of terminal lineages, $k$, against the relative-extinction rate used in the simulation. Trees were simulated under a constant-rate birth-death process using the parameters summarized in Table S.32, The cells within each panel are colored to reflect the $95 \%$ quantile of the magnitude of the inferred shift in relative-extinction rate when a two-rate model is incorrectly identified (see Shift Magnitude legend). In order to accommodate the bimodal distribution of rate shifts, the magnitude of each relative-extinction-rate shift was computed as the ratio of the higher rate to the lower rate, regardless of which rate corresponded to the background-rate category. The average $95 \%$ quantile of the magnitude of shifts in relative-extinction rate shift is summarized in the lower right of each panel. 
bioRxiv preprint doi: https://doi.org/10.1101/011452; this version posted November 15, 2014. The copyright holder for this preprint (which was not certified by peer review) is the author/funder, who has granted bioRxiv a license to display the preprint in perpetuity. It is made available under aCC-BY-ND 4.0 International license.

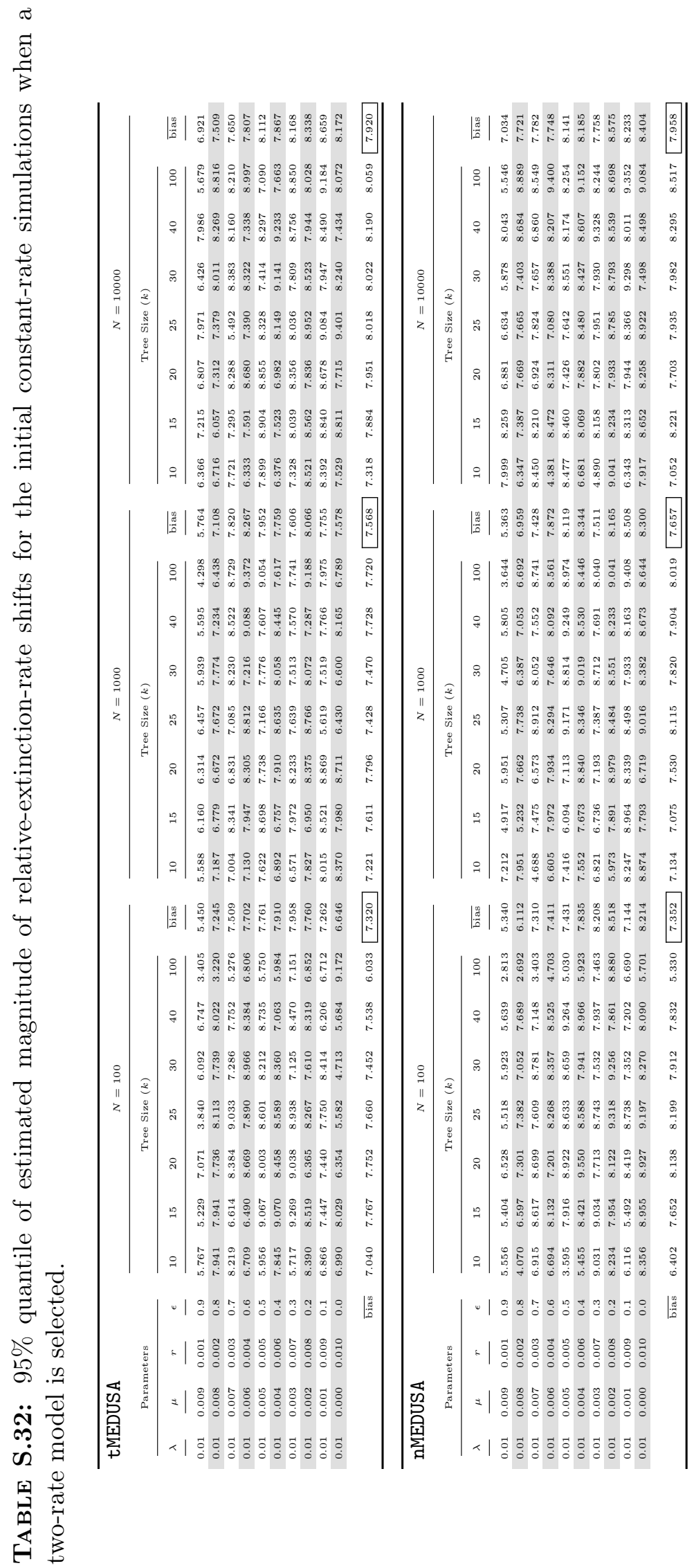




\section{Supplementary References}

Alfaro, M. E., F. Santini, C. Brock, H. Alamillo, A. Dornburg, D. L. Rabosky, G. Carnevale, and L. J. Harmon. 2009. Nine exceptional radiations plus high turnover explain species diversity in jawed vertebrates. Proceedings of the National Academy of Sciences 106:13410-13414.

Antonelli, A. and I. Sanmartin. 2011. Mass extinction, gradual cooling, or rapid radiation? reconstructing the spatiotemporal evolution of the ancient angiosperm genus Hedyosmum (Chloranthaceae) using empirical and simulated approaches. Systematic Biology 60:596-615.

Arakaki, M., P.-A. Christin, R. Nyffeler, A. Lendel, U. Eggli, R. M. Ogburn, E. Spriggs, M. J. Moore, and E. J. Edwards. 2011. Contemporaneous and recent radiations of the world's major succulent plant lineages. Proceedings of the National Academy of Sciences 108:8379-8384.

Baker, W. J. and T. L. P. Couvreur. 2013. Global biogeography and diversification of palms sheds light on the evolution of tropical lineages. II. Diversification history and origin of regional assemblages. Journal of Biogeography 40:286-298.

Barker, F. K., K. J. Burns, J. Klicka, S. M. Lanyon, and I. J. Lovette. 2013. Going to extremes: Contrasting rates of diversification in a recent radiation of New World Passerine birds. Systematic Biology 62:298-320.

Beaulieu, J. M. and M. J. Donoghue. 2013. Fruit evolution and diversification of Campanulid angiosperms. Evolution 67:3132-3144.

Blankers, T., T. M. Townsend, K. Pepe, T. W. Reeder, and J. J. Wiens. 2013. Contrasting globalscale evolutionary radiations: phylogeny, diversification, and morphological evolution in the major clades of iguanian lizards. Biological Journal of the Linnean Society 108:127-143.

Castroviejo-Fisher, S., J. M. Guayasamin, A. Gonzalez-Voyer, and C. Vila. 2014. Neotropical diversification seen through glassfrogs. Journal of Biogeography 41:66-80.

Chakrabarty, P., M. P. Davis, W. L. Smith, Z. H. Baldwin, and J. S. Sparks. 2011. Is sexual selection driving diversification of the bioluminescent ponyfishes (Teleostei: Leiognathidae)? Molecular Ecology 20:2818-2834. 
Chenoweth, L. B. and M. P. Schwarz. 2011. Biogeographical origins and diversification of the exoneurine allodapine bees of Australia (Hymenoptera, Apidae). Journal of Biogeography 38:14711483.

Claramunt, S., E. P. Derryberry, R. T. Brumfield, and J. V. Remsen, Jr. 2012. Ecological opportunity and diversification in a continental radiation of birds: Climbing adaptations and cladogenesis in the Furnariidae. American Naturalist 179:649-666.

Cowman, P. F. and D. R. Bellwood. 2011. Coral reefs as drivers of cladogenesis: Expanding coral reefs, cryptic extinction events, and the development of biodiversity hotspots. Journal oif Evolutionary Biology 24:2543-2562.

Davis, A. M., P. J. Unmack, B. J. Pusey, J. B. Johnson, and R. G. Pearson. 2012. Marine-freshwater transitions are associated with the evolution of dietary diversification in terapontid grunters (Teleostei: Terapontidae). Journal of Evolutionart Biology 25:1163-1179.

Derryberry, E. P., S. Claramunt, G. Derryberry, R. T. Chesser, J. Cracraft, A. Aleixo, J. PerezEman, J. V. Remsen, Jr., and R. T. Brumfield. 2011. Lineage diversification and morphological evolution in a large-scale continental radiation: the Neotrophical overbirds and woodcreepers (Aves: Furnariidae). Evolution 65:2973-2986.

Drummond, C. S., R. J. Eastwood, S. T. S. Miotto, and C. E. Hughes. 2012. Multiple continental radiations and correlates of diversification in Lupinus(Leguminosae): Testing for key innovation with incomplete taxon sampling. Systematic Biology 61:443-460.

Escudero, M., A. L. Hipp, M. J. Waterway, and L. M. Valente. 2012. Diversification rates and chromosome evolution in the most diverse angiosperm genus of the temperate zone (Carex, Cyperaceae). Molecular Phylogenetic and Evolution 63:650-655.

Escudero, M. and A. Hipp. 2013. Shifts in diversification rates and clade ages explain species richness in higher-level sedge taxa (Cyperaceae). American Journal of Botany 100:2403-2411.

Fiz-Palacios, O., H. Schneider, J. Heinrichs, and V. Savolainen. 2011. Diversification of land plants: Insights from a family-level phylogenetic analysis. BMC Evolutionart Biology 11. 
Fiz-Palacios, O., M. Romeralo, A. Ahmadzadeh, S. Weststrand, P. E. Ahlberg, and S. Baldauf. 2013. Did terrestrial diversification of Amoebas (Amoebozoa) occur in synchrony with land plants? PLoS ONE 8.

Frederich, B., L. Sorenson, F. Santini, G. J. Slater, and M. E. Alfaro. 2013. Iterative ecological radiation and convergence during the evolutionary history of damselfishes (Pomacentridae). American Naturalist 181:94-113.

Giribet, G. and G. D. Edgecombe. 2013. Stable phylogenetic patterns in scutigeromorph centipedes (Myriapoda : Chilopoda : Scutigeromorpha): dating the diversification of an ancient lineage of terrestrial arthropods. Invertebrate Systematics 27.

Givnish, T. J., M. H. J. Barfuss, B. Van Ee, R. Riina, K. Schulte, R. Horres, P. A. Gonsiska, R. S. Jabaily, D. M. Crayn, J. A. C. Smith, K. Winter, G. K. Brown, T. M. Evans, B. K. Holst, H. Luther, W. Till, G. Zizka, P. E. Berry, and K. J. Sytsma. 2014. Adaptive radiation, correlated and contingent evolution, and net species diversification in Bromeliaceae. Molecular Phylogenetic and Evolution 71:55-78.

Groom, S. V. C., M. I. Stevens, and M. P. Schwarz. 2013. Diversification of Fijian halictine bees: Insights into a recent island radiation. Molecular Phylogenetic and Evolution 68:582-594.

Guzman, B., M.-A. Lachance, and C. M. Herrera. 2013. Phylogenetic analysis of the angiospermfloricolous insect-yeast association: Have yeast and angiosperm lineages co-diversified? Molecular Phylogenetic and Evolution 68:161-175.

Heikkila, M., L. Kaila, M. Mutanen, C. Pena, and N. Wahlberg. 2012. Cretaceous origin and repeated tertiary diversification of the redefined butterflies. Proceedings of the Royal Society B: Biological Sciences 279:1093-1099.

Jetz, W., G. H. Thomas, J. B. Joy, K. Hartmann, and A. O. Mooers. 2012. The global diversity of birds in space and time. Nature 491:444-448.

Johnson, M. T. J., R. G. FitzJohn, S. D. Smith, M. D. Rausher, and S. P. Otto. 2011. Loss of sexual recombination and segregation is associated with increased diversification in evening Primroses. Evolution 65:3230-3240. 
Koenen, E. J. M., J. M. de Vos, G. W. Atchison, M. F. Simon, B. D. Schrire, E. R. de Souza, L. P. de Queiroz, and C. E. Hughes. 2013. Exploring the tempo of species diversification in legumes. South African Journal of Botany 89:19-30.

Litman, J. R., B. N. Danforth, C. D. Eardley, and C. J. Praz. 2011. Why do leafcutter bees cut leaves? New insights into the early evolution of bees. Proceedings of the Royal Society B: Biological Sciences 278:3593-3600.

Litman, J. R., C. J. Praz, B. N. Danforth, T. L. Griswold, and S. Cardinal. 2013. Origins, evolution, and diversification of cleptoparasitic lineages in long-tongued bees. Evolution 67:2982-2998.

Leslie, A. B., J. M. Beaulieu, P. R. Crane, and M. J. Donoghue. 2013. Explaining the distribution of breeding and dispersal syndromes in conifers. Proceedings of the Royal Society B: Biological Sciences 280 .

Matthews, L. J., C. Arnold, Z. Machanda, and C. L. Nunn. 2011. Primate extinction risk and historical patterns of speciation and extinction in relation to body mass. Proceedings of the Royal Society B: Biological Sciences 278:1256-1263.

McLeish, M. 2011. Speciation in gall-inducing thrips on Acacia in arid and non-arid areas of Australia. Journal of Arid Environments 75:793-801.

McMahan, C. D., P. Chakrabarty, J. S. Sparks, W. L. Smith, and M. P. Davis. 2013. Temporal patterns of divesification across global cichlid biodiversity (Acanthomorpha: Cichlidae). PLoS ONE 8.

Morales-Hojas, R. and J. Vieira. 2012. Phylogenetic patterns of geographical and ecological diversification in the subgenus Drosophila. PLoS ONE 7.

Moreau, C. S. and C. D. Bell. 2013. Testing the museum versus cradle tropical biological diversity hypothesis: Phylogeny, diversification, and ancestral biogeographic range evolution of the ants. Evolution 67:2240-2257.

Murray, E. A., A. E. Carmichael, and J. M. Heraty. 2013. Ancient host shifts followed by host conservatism in a group of ant parasitoids. Proceedings of the Royal Society B: Biological Sciences 280. 
Nagy, L. G., J. Hazi, B. Szappanos, S. Kocsube, B. Balint, G. Rakhely, C. Vagvoelgyi, and T. Papp. 2012. The evolution of defense mechanisms correlate with the explosive diversification of autodigesting Coprinellus mushrooms (Agaricales, Fungi). Systematic Biology 61:595-607.

Near, T. J., A. Dornburg, K. L. Kuhn, J. T. Eastman, J. N. Pennington, T. Patarnello, L. Zane, D. A. Fernandez, and C. D. Jones. 2012. Ancient climate change, antifreeze, and the evolutionary diversification of Antarctic fishes. Proceedings of the National Academy of Sciences 109:34343439.

Near, T. J., A. Dornburg, R. I. Eytan, B. P. Keck, W. L. Smith, K. L. Kuhn, J. A. Moore, S. A. Price, F. T. Burbrink, M. Friedman, and P. C. Wainwright. 2013. Phylogeny and tempo of diversification in the superradiation of spiny-rayed fishes. Proceedings of the National Academy of Sciences 110:12738-12743.

Price, S. L., S. Powell, D. J. C. Kronauer, L. A. P. Tran, N. E. Pierce, and R. K. Wayne. 2014. Renewed diversification is associated with new ecological opportunity in the Neotropical turtle ants. Journal of Evolutionary Biology 27:242-258.

Pyron, R. A. and F. T. Burbrink. 2012. Extinction, ecological opportunity, and the origins of global snake diversity. Evolution 66:163-178.

Rabosky, D. L., G. J. Slater, and M. E. Alfaro. 2012. Clade age and species richness are decoupled across the Eukaryotic Tree of Life. PLoS Biology 10.

Roncal, J., F. Kahn, B. Millan, T. L. P. Couvreur, and J.-C. Pintaud. 2013. Cenozoic colonization and diversification patterns of tropical American palms: evidence from Astrocaryum (Arecaceae). Botanical Journal of the Linnean Society 171:120-139.

Ryberg, M. and P. B. Matheny. 2012. Asynchronous origins of ectomycorrhizal clades of Agaricales. Proceedings of the Royal Society B: Biological Sciences 279:2003-2011.

Santini, F., L. J. Harmon, G. Carnevale, and M. E. Alfaro. 2009. Did genome duplication drive the origin of teleosts? A comparative study of diversification in ray-finned fishes. BMC Evolutionary Biology 9. 
Santini, F., G. Carnevale, and L. Sorenson. 2013a. First molecular scombrid timetree (Percomorpha: Scombridae) shows recent radiation of tunas following invasion of pelagic habitat. Italian Journal of Zoology 80:210-221.

Santini, F., M. T. T. Nguyen, L. Sorenson, T. B. Waltzek, J. W. L. Alfaro, J. M. Eastman, and M. E. Alfaro. 2013b. Do habitat shifts drive diversification in teleost fishes? An example from the pufferfishes (Tetraodontidae). Journal of Evolutionary Biology 26:1003-1018.

Santini, F., L. Sorenson, and M. E. Alfaro. 2013c. A new multi-locus timescale reveals the evolutionary basis of diversity patterns in triggerfishes and filefishes (Balistidae, Monacanthidae; Tetraodontiformes). Molecular Phylogenetic and Evolution 69:165-176.

Schenk, J. J., K. C. Rowe, and S. J. Steppan. 2013. Ecological opportunity and incumbency in the diversification of repeated continental colonizations by Muroid rodents. Systematic Biology $62: 837-864$.

Schirrmeister, B. E., J. M. de Vos, A. Antonelli, and H. C. Bagheri. 2013. Evolution of multicellularity coincided with increased diversification of cyanobacteria and the Great Oxidation Event. Proceedings of the National Academy of Sciences 110:1791-1796.

Schweizer, M., O. Seehausen, and S. T. Hertwig. 2011. Macroevolutionary patterns in the diversification of parrots: effects of climate change, geological events and key innovations. Journal of Biogeography 38:2176-2194.

Slater, G. J., S. A. Price, F. Santini, and M. E. Alfaro. 2010. Diversity versus disparity and the radiation of modern cetaceans. Proceedings of the Royal Society B: Biological Sciences 277:30973104 .

Van Velzen, R., N. Wahlberg, M. S. M. Sosef, and F. T. Bakker. 2013. Effects of changing climate on species diversification in tropical forest butterflies of the genus Cymothoe (Lepidoptera: Nymphalidae). Biological Journal of the Linnean Society 108:546-564.

Wahlberg, N., C. W. Wheat, and C. Pena. 2013. Timing and patterns in the taxonomic diversification of Lepidoptera (butterflies and moths). PLoS ONE 8. 
Wiegmann, B. M., M. D. Trautwein, I. S. Winkler, N. B. Barr, J.-W. Kim, C. Lambkin, M. A. Bertone, B. K. Cassel, K. M. Bayless, A. M. Heimberg, B. M. Wheeler, K. J. Peterson, T. Pape, B. J. Sinclair, J. H. Skevington, V. Blagoderov, J. Caravas, S. N. Kutty, U. Schmidt-Ott, G. E. Kampmeier, F. C. Thompson, D. A. Grimaldi, A. T. Beckenbach, G. W. Courtney, M. Friedrich, R. Meier, and D. K. Yeates. 2011. Episodic radiations in the fly tree of life. Proceedings of the National Academy of Sciences 108:5690-5695.

Xi, Z., B. R. Ruhfel, H. Schaefer, A. M. Amorim, M. Sugumaran, K. J. Wurdack, P. K. Endress, M. L. Matthews, P. F. Stevens, S. Mathews, and C. C. Davis. 2012. Phylogenomics and a posteriori data partitioning resolve the Cretaceous angiosperm radiation Malpighiales. Proceedings of the National Academy of Sciences 109:17519-17524.

Yu, W., J. Xu, Y. Wu, and G. Yang. 2012. A comparative study of mammalian diversification pattern. International Journal of Biological Sciences 8. 